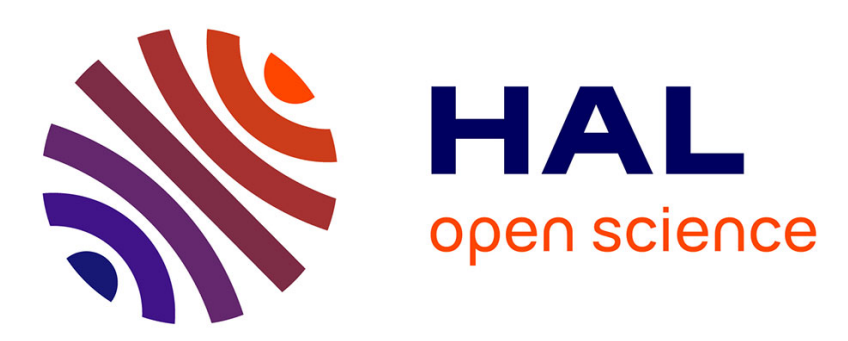

\title{
FFT-based simulations of slip and kink bands formation in 3D polycrystals: Influence of strain gradient crystal plasticity
}

\author{
Aldo Marano, Lionel Gélébart, Samuel Forest
}

\section{- To cite this version:}

Aldo Marano, Lionel Gélébart, Samuel Forest. FFT-based simulations of slip and kink bands formation in 3D polycrystals: Influence of strain gradient crystal plasticity. Journal of the Mechanics and Physics of Solids, 2021, 149, pp.104295. 10.1016/j.jmps.2021.104295 . hal-03372378

\section{HAL Id: hal-03372378 \\ https://hal.science/hal-03372378}

Submitted on 16 Oct 2021

HAL is a multi-disciplinary open access archive for the deposit and dissemination of scientific research documents, whether they are published or not. The documents may come from teaching and research institutions in France or abroad, or from public or private research centers.
L'archive ouverte pluridisciplinaire HAL, est destinée au dépôt et à la diffusion de documents scientifiques de niveau recherche, publiés ou non, émanant des établissements d'enseignement et de recherche français ou étrangers, des laboratoires publics ou privés. 


\title{
FFT-based simulations of slip and kink bands formation in 3D polycrystals: influence of strain gradient crystal plasticity
}

\author{
Aldo Marano ${ }^{\mathrm{a}, \mathrm{b}}$, Lionel Gélébart ${ }^{\mathrm{a}, *}$, Samuel Forest ${ }^{\mathrm{b}}$ \\ ${ }^{a}$ DEN-Service de Recherches Métallurgiques Appliquées, CEA, Université Paris-Saclay, F-91191, Gif-sur-Yvette, France \\ ${ }^{b}$ MINES ParisTech, PSL Research University, MAT - Centre des matériaux, CNRS UMR 7633, BP 879103 Evry, France
}

\begin{abstract}
The ability of softening strain gradient plasticity to simulate intragranular plastic slip localization modes called slip and kink bands, at is investigated at incipient plasticity. A strain gradient crystal plasticity model based on a quadratic energy contribution of the Nye tensor has been implemented within the massively parallel FFT-based solver AMITEX_FFTP. It is shown that a classical evaluation of the double curl operator does not properly account for the backstress induced by GNDs in slip and kink bands. Consequently, a better suited differentiation operator is proposed for this purpose. Besides, four practical implementations of interface conditions at grain boundaries are implemented and discussed. It is demonstrated that these conditions have a strong influence on GND induced hardening but weakly affect the formation of slip localization patterns.

A theoretical and numerical study of an idealized kink band shows that this modeling framework, contrary to classical crystal plasticity models, is able to capture their essential physical features. A quantitative analysis of high resolution 3D polycrystalline simulations, based on imaged processing and a peak detection on plastic slip profiles, is applied to show that gradient effects strongly influence the mechanisms of selection between slip and kink banding, to the favor of the first. In particular, some kink bands are shown to decompose into an homogeneous distribution of parallel slip bands of finite length. Finally, the competition between structural effects and energetic cost of lattice curvature in the formation of localized slip bands networks in polycrystals is discussed. The present results demonstrate the relevance of softening crystal plasticity modeling including an energetic contribution of GND density to accurately capture slip localization in polycrystals.
\end{abstract}

Keywords: Gradient crystal plasticity, Intragranular localization, Slip bands, Kink bands, FFT simulations

\section{Introduction}

The heterogeneous nature of plastic slip is the subject of a long-standing interest in the material science community, as a key to connect the micro-scale physics of dislocations to the macroscopical behavior of crystalline solids. Essential works have precisely described the typology of the various localization phenomena associated to this process (Jaoul, 1964; Neuhäuser, 1983). Among them, the common single-slip deformation occurring at incipient plasticity can yield two different types of localization bands, schematically represented in fig. 1. Slip bands (SBs) (fig. 1(a)) are sharp structures formed by intense dislocation glide on a few neighboring crystallographic planes. They are parallel to the active slip plane and are the main

\footnotetext{
* Corresponding author

Email addresses: aldo.marano@cea.fr (Aldo Marano), lionel.gelebart@cea.fr (Lionel Gélébart), samuel.forest@mines-paristech.fr (Samuel Forest)
} 
signature of plastic slip in crystals. On the other hand, Kink bands (KBs) (fig. 1-(b)) form orthogonal to the glide direction. Their kinematics results in a strong lattice rotation within the band with respect to the surrounding crystal. They are bounded by high lattice curvature boundaries formed by geometrically necessary dislocation (GND) walls and have a much larger characteristic width than SB. In addition, as evidenced by the recent observations of di Gioacchino and da Fonseca (2015), their structure is characterized by a dense distribution of short SB along the slip plane normal direction. Less common than SBs, KBs are known since the pioneering work of Orowan (1942). They are reported mostly in strongly plastically anisotropic crystals such as ice (Mansuy et al., 2001; Montagnat et al., 2011; Wilson et al., 1986), Zinc (Gilman, 1954; Hagihara et al., 2016a), Magnesium (Hagihara et al., 2016b) or Titanium (Churchman, 1955). They have also been noticed in iron crystals (Jaoul, 1961) and as crack-tip deformation modes in notched single crystals (Crone and Shield, 2001; Kysar and Briant, 2002; Patil et al., 2009; Flouriot et al., 2003). Hence, they arise to accommodate strong strain incompatibilities when only one slip system can be activated.

SB and KB are material-based localization instabilities triggered by softening mechanisms at the dislocation scale (Estrin and Kubin, 1986; Brechet et al., 1993). In some materials, such as irradiated (Sharp, 1974; Onimus et al., 2004; Byun et al., 2006), hyper-quenched (Mori and Meshii, 1969; Bapna and Meshii, 1974) or hydrogen-charged metals (Aubert et al., 2012), these mechanisms are particularly strong. As a result, they induce the formation of very intense SBs, promoting failure and reducing metals ductility. This concern has motivated numerous modeling efforts, relying on dislocation-based softening classical crystal plasticity (CCP) models to simulate such plastic slip localization (Zhang et al., 2010; Barton et al., 2013; Patra and McDowell, 2016; Xiao et al., 2015; Erinosho and Dunne, 2015; Hure et al., 2016). These models effectively lead to the formation of SBs and KBs within polycrystalline simulations. However, SBs and KBs arise in this framework as two equivalent bifurcation modes of CCP equations. Asaro and Rice (1977) demonstrated this classical result by conducting a bifurcation analysis for an homogeneous crystal undergoing single slip. The validity of this $\mathrm{SB} / \mathrm{KB}$ equivalence has recently been extended to polycrystalline simulations computed with most CCP models (Marano et al., 2019). It results in a purely structural selection mechanism between $\mathrm{SB}$ or KB. That is to say, between the SB and the KB, the one having the best suited geometrical orientation to locally accommodate the imposed strain forms, and no other parameter influences the selection. This leads systematically to comparable amounts of these two localization modes in the simulations, as in most crystal structure slip and kink planes are similarly distributed in the microstructure. Yet, in reality, $\mathrm{SB}$ are overwhelmingly predominant in real materials. Moreover, the respective formation processes of SBs and KBs strongly differ from a physical point a view. For instance, a KB formation requires the activation of much more dislocation sources than a SB formation. This strongly suggests to include other physical parameters in the modeling, to account for these differences. Consequently, CCP models cannot accurately predict intra-granular plastic slip localization (i.e. slip and kink banding).

It has been evidenced by means of Cosserat plasticity theory by Forest (1998), that accounting for an energetic contribution of lattice curvature allows to break the equivalence between the two modes. Likewise, strain gradient plasticity (SGP) theories that enrich the classical local formulation by accounting for Nye's geometrically necessary dislocation (GND) density tensor, should provide a natural framework to address this shortcoming of CCP. Indeed, according to Nye's formula (Nye, 1953), Nye's tensor is linked to crystal lattice curvature, providing thus a physical measure allowing to distinguish SBs from KBs. Extensive developments in the past decades have spawned numerous formulations of these theories (Fleck and Hutchinson, 1997; Acharya and Bassani, 2000; Gurtin, 2002; Wulfinghoff et al., 2015; Cordero et al., 2010; Kaiser and Menzel, 2019) but have been mostly focused on modeling size effects associated to plasticity. A few studies have presented simulations of SB/KB formation in softening single crystal structures (Ling et al., 2018; Scherer et al., 2019) but they rely on a simplified theory accounting for the gradient of a cumulative scalar slip variable, designed to efficiently regularize both modes with a single length scale. In doing so, they naturally preserve the non-physical SB/KB equivalence. In fact, the promising potential of SGP models to improve the physical relevance of SBs and KBs formation modeling has not been explored yet, and therefore needs a detailed focus.

This paper is dedicated to this task. Using a simple model based on the SGP theory of Gurtin (2002), 
accounting for the full GND density tensor, its aim is to evidence, by comparison with CCP, the influence of SGP on the modeling of individual slip and kink bands, as well as on the development of complex localization patterns in 3D polycrystals. High resolution simulations are needed to analyze the influence of the microstructure on their formation mechanisms. To perform such simulations, a previous work conducted by the present authors in the framework of CCP models (Marano et al., 2019) has relied on the efficiency of massively parallel FFT-based solvers. In the present work, we take advantage of their recent extension to Gurtin's theory by Lebensohn and Needleman (2016), and to the Nye's tensor based Field Dislocation Mechanics (Berbenni et al., 2014; Brenner et al., 2014; Eloh et al., 2019) to apply the same strategy to SGP. However, these authors did not study the influence of their implementation on the numerical modeling of slip localization modes, especially with respect to the differentiation operators used to compute plastic strain gradients, and higher order interface conditions at grain boundaries. A detailed discussion of this matter is also provided in the present work.

This paper is organized as follows. Section 2 presents the kinematics, balance and constitutive equations of the SGP model and their application to individual SB/KB modeling. Section 3 discusses its implementation within the massively parallel FFT-based solver, AMITEX_FFTP, and associated numerical issues. Thereupon, section 4, resp. section 5 present the application of the model to slip localization simulations in single crystals, resp. in polycrystals. Finally, the influence of grain boundary interface conditions, the numerical implementation and the physics of slip/kink bands formation within SGP models are discussed in section 6 .

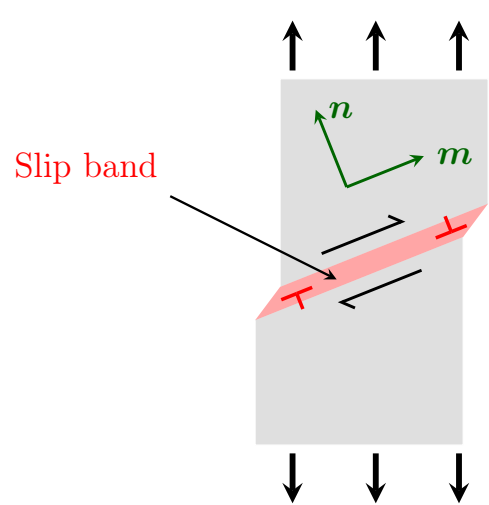

(a)

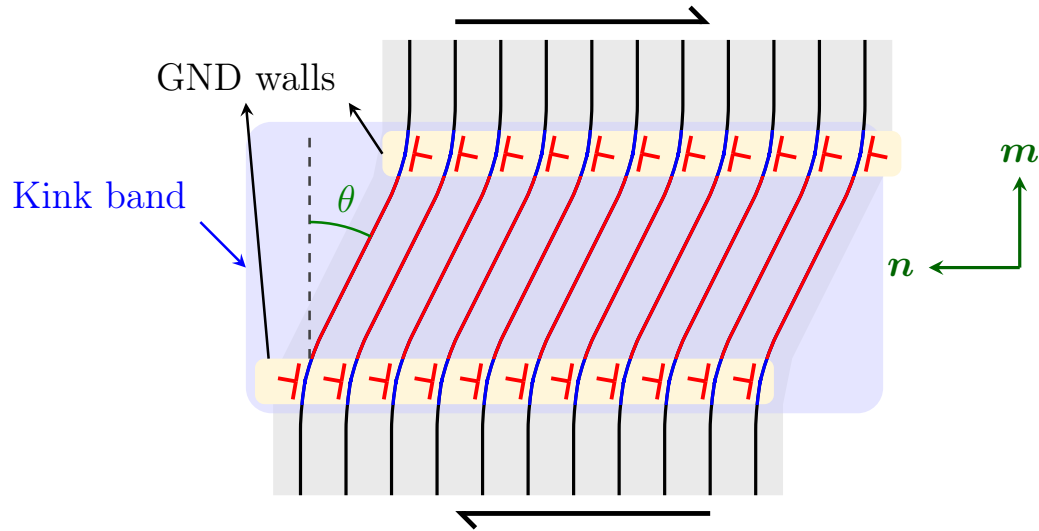

(b)

Figure 1: Schematic representation of the two single slip localization modes: the slip band and the kink band. $\boldsymbol{m}$ and $\boldsymbol{n}$ are resp. the glide direction and normal to slip plane direction. The red lines in the kink band represent densely distributed slip bands. $\theta$ represents the lattice rotation angle inside the band. The high lattice curvature boundaries and associated GND wall structures are highlighted in light yellow.

\section{Notation}

Throughout this paper, $a, \boldsymbol{a}, \underline{\boldsymbol{a}}, \boldsymbol{a}, \underline{\underline{\boldsymbol{A}}}$ denote respectively scalars, vectors, second, third and fourth order tensors. $\left(\boldsymbol{e}_{1}, \boldsymbol{e}_{2}, \boldsymbol{e}_{3}\right)$ denote the classical Cartesian basis of the euclidean space. $\dot{a}$ denotes the time derivative of the variable $a$. The following definition of the curl operator for second order tensors is adopted here:

$$
\operatorname{curl}(\underline{\boldsymbol{a}})=\epsilon_{j m s} a_{i m, s} \boldsymbol{e}_{i} \otimes \boldsymbol{e}_{j}
$$

where $\underset{\sim}{\boldsymbol{\epsilon}}$ is the Levi-Civita third order permutation tensor. 


\section{Strain gradient crystal plasticity model}

\subsection{The curl $\left(H^{p}\right)$ model}

As stated in the introduction, the present work is focused on the development of slip localization at incipient plasticity, which occurs at very low local strains. For this reason, our study is limited to a crystal plasticity theory in the context of small deformations. It relies on a plasticity theory that includes the curl of the plastic deformation tensor as non-local variable, initially proposed by Gurtin (2002) and Svendsen (2002), that we recall here. For the sake of brevity, the equations are directly provided here ${ }^{1}$.

The displacement gradient tensor $\nabla \underline{\boldsymbol{u}}$ is decomposed as the sum of an elastic $\underline{\boldsymbol{H}}^{e}$ and a plastic part $\underline{\boldsymbol{H}}^{p}$ :

$$
\underline{\boldsymbol{H}}=\nabla \underline{\boldsymbol{u}}=\underline{\boldsymbol{H}}^{e}+\underline{\boldsymbol{H}}^{p}
$$

The elastic strain tensor $\underline{\boldsymbol{\varepsilon}}^{e}$ is defined as the symmetric part of $\underline{\boldsymbol{H}}^{e}$. The plastic deformation rate is determined by the shearing rate of the $N_{s}$ slip systems of the crystal, characterized by their slip plane normal direction $\boldsymbol{n}^{s}$ and glide direction $\boldsymbol{m}^{s}$, through the relation:

$$
\underline{\dot{\boldsymbol{H}}}^{p}=\sum_{s}^{N_{s}} \dot{\gamma}^{s} \underline{\boldsymbol{\mu}}^{s}
$$

where $\boldsymbol{\mu}^{s}=\boldsymbol{m}^{s} \otimes \boldsymbol{n}^{s}$ is the Schmid orientation tensor of slip system $s$. Additionally, the following useful quantities are defined: the effective cumulative plastic strain $p$ through eq. (3) and the cumulative plastic slip on slip system $s, \gamma_{\text {cum }}^{s}$, through eq. (4).

$$
\begin{aligned}
p & =\int_{0}^{t} \sqrt{\underline{\dot{\boldsymbol{H}}}^{p}: \underline{\dot{\boldsymbol{H}}}^{p}} d t \\
\gamma_{\text {cum }}^{\text {s }} & =\int_{0}^{t}\left|\dot{\gamma}^{s}\right| d t
\end{aligned}
$$

Then, let us denote by $\underline{\omega}_{e}$, the skew-symmetric part of the $\underline{\boldsymbol{H}}^{e}$, and $\stackrel{\times}{\omega}$ the associated axial vector. At small deformations, $\underline{\omega}_{e}$ is linked to the lattice rotation angle $\theta$ by the relation $\theta=\|\stackrel{\times}{\omega}\|$. Besides, for small angles $|\theta| \ll 1$, and in a base $\left(\boldsymbol{e}_{1}^{\prime}, \boldsymbol{e}_{2}^{\prime}, \boldsymbol{e}_{3}^{\prime}\right)$ where $\boldsymbol{e}_{3}^{\prime}$ is aligned with the rotation axis, $\omega$ can be written as a function of $\theta$ as follows:

$$
\underline{\omega}_{e} \approx \theta\left(\boldsymbol{e}_{2}^{\prime} \otimes \boldsymbol{e}_{1}^{\prime}-\boldsymbol{e}_{1}^{\prime} \otimes \boldsymbol{e}_{2}^{\prime}\right)
$$

As a consequence of eq. (1), $\operatorname{curl}(\underline{\boldsymbol{H}})=\operatorname{curl}(\nabla \underline{\boldsymbol{u}})=0 \Rightarrow \operatorname{curl}\left(\underline{\boldsymbol{H}}^{e}\right)=-\operatorname{curl}\left(\underline{\boldsymbol{H}}^{p}\right)$. As $\underline{\boldsymbol{H}}^{p}$ and $\underline{\boldsymbol{H}}^{e}$ are generally incompatible, $\operatorname{curl}\left(\underline{\boldsymbol{H}}^{e}\right)$ and $-\operatorname{curl}\left(\underline{\boldsymbol{H}}^{p}\right)$ do not vanish. This quantity is the dislocation (GND) density tensor, or Nye tensor (Nye, 1953; Steinmann, 1996; Cermelli and Gurtin, 2001), and is denoted by $\underline{\alpha}:$

$$
\underline{\boldsymbol{\alpha}}=-\operatorname{curl}\left(\underline{\boldsymbol{H}}^{p}\right)=-\epsilon_{j m s} H_{i m, s}^{p} \boldsymbol{e}_{i} \otimes \boldsymbol{e}_{j}
$$

The continuum internal power density $p_{i}$ and contact power density $p_{c}$ take the following form:

$$
p_{i}=\underline{\boldsymbol{\sigma}}: \nabla \underline{\boldsymbol{u}}+\underline{\boldsymbol{s}}: \dot{\boldsymbol{H}}^{p}+\underline{\boldsymbol{M}}: \operatorname{curl}\left(\underline{\dot{\boldsymbol{H}}}^{p}\right) \quad p_{c}=\boldsymbol{t}: \dot{\boldsymbol{u}}+\underline{\boldsymbol{m}}: \underline{\dot{\boldsymbol{H}}}^{p}
$$

$\underline{\boldsymbol{\sigma}}$ is the Cauchy stress tensor, $\underline{\boldsymbol{s}}$, work-conjugate to $\underline{\boldsymbol{H}}^{p}$, is the micro-stress tensor, and $\underline{\boldsymbol{M}}$, work-conjugate to $\operatorname{curl}\left(\underline{\boldsymbol{H}}^{p}\right)$, is the double stress tensor. $\boldsymbol{t}$ is the classical traction tensor and $\underline{\boldsymbol{m}}$ the double-traction vector.

\footnotetext{
${ }^{1}$ for a more detailed derivation of the theory, the reader is referred to Cordero et al. (2010)
} 
In the absence of external body forces and inertial forces, the principle of virtual power yields the following balance equations, eqs. (8) and (9), and boundary conditions, eqs. (10) and (11):

$$
\begin{array}{lllll}
\operatorname{div}(\underline{\boldsymbol{\sigma}})=0 & \sigma_{i j, j}=0 & (8) & \boldsymbol{t}=\underline{\boldsymbol{\sigma}} \cdot \boldsymbol{n} & t_{i}=\sigma_{i j} n_{j} \\
\operatorname{curl}(\underline{\boldsymbol{M}})+\underline{\boldsymbol{s}}=0 & \epsilon_{j k l} M_{i k, l}+s_{i j}=0 & (9) & \underline{\boldsymbol{m}}=\underline{\boldsymbol{M}} \cdot \underline{\boldsymbol{\epsilon}} \cdot \boldsymbol{n} & m_{i j}=M_{i k} \epsilon_{k j l} n_{l}
\end{array}
$$

On a boundary surface or interface $\mathcal{S}$ of the domain, simple higher order boundary conditions corresponding to a vanishing power of the generalized stresses can be considered. Equations (12) and (13) present respectively the microhard boundary condition, which requires that the plastic slip vanishes on $\mathcal{S}$ and the microfree boundary condition, which requires that the generalized traction vanishes on $\mathcal{S}$.

$$
\begin{array}{ll}
\underline{\boldsymbol{H}}^{p}(\boldsymbol{X})=0 & \forall \boldsymbol{x} \in \mathcal{S} \\
\underline{\boldsymbol{m}}(\boldsymbol{x})=\underline{\boldsymbol{M}}(\boldsymbol{x}) \cdot \boldsymbol{\boldsymbol { \epsilon }} \cdot \boldsymbol{n}=0 & \forall \boldsymbol{x} \in \mathcal{S}
\end{array}
$$

The Helmholtz free energy density $\psi$ is assumed here to be a function: $\psi=\psi\left(\underline{\boldsymbol{\varepsilon}}^{e}, \operatorname{curl}\left(\underline{\boldsymbol{H}}^{p}\right), \gamma_{c u m}^{s}\right)$. Substituting eqs. (1) and (7) in the Clausius-Duhem inequality, $p_{i}-\rho \dot{\psi} \geq 0$, yields:

$$
\left(\underline{\boldsymbol{\sigma}}-\rho \frac{\partial \psi}{\partial \underline{\boldsymbol{\varepsilon}}^{e}}\right): \underline{\boldsymbol{\varepsilon}}^{e}+(\underline{\boldsymbol{\sigma}}+\underline{\boldsymbol{s}}): \underline{\dot{\boldsymbol{H}}}^{p}+\left(\underline{\boldsymbol{M}}-\rho \frac{\partial \psi}{\partial \operatorname{curl}\left(\underline{\boldsymbol{H}}^{p}\right)}\right): \operatorname{curl}\left(\underline{\dot{\boldsymbol{H}}}^{p}\right)-\sum_{s=1}^{N} \rho \frac{\partial \psi}{\partial \gamma_{\text {cum }}^{s}} \dot{\gamma}_{\text {cum }}^{s} \geq 0
$$

Then, it is assumed that the work associated to elastic deformation and to $\operatorname{curl}\left(\underline{\boldsymbol{H}}^{p}\right)$ is non dissipative processes, and contribute through a simple quadratic potential, such that:

$$
\rho \psi\left(\underline{\boldsymbol{\varepsilon}}^{e}, \operatorname{curl}\left(\underline{\boldsymbol{H}}^{p}\right), \gamma_{\text {cum }}^{s}\right)=\frac{1}{2} \underline{\boldsymbol{\varepsilon}}^{e}: \underline{\underline{\boldsymbol{\Lambda}}}: \underline{\boldsymbol{\varepsilon}}^{e}+\frac{1}{2} \pi_{0} \lambda^{2} \operatorname{curl}\left(\underline{\boldsymbol{H}}^{p}\right): \operatorname{curl}\left(\underline{\boldsymbol{H}}^{p}\right)+\sum_{s}^{N_{s}} \psi_{s}\left(\gamma_{\text {cum }}^{s}\right)
$$

where the $\psi_{s}$ are functions representing the dependence of the free energy on the hardening variables $\gamma_{\text {cum }}^{s}$, and where $\underline{\underline{\boldsymbol{\Lambda}}}$ is the fourth-order tensor of elastic moduli, $\lambda$ and $\pi_{0}$ are material parameters with respectively the dimension of length and stress. Hence, the associated non dissipative terms in eq. (14) must vanish, which yields the following linear constitutive relations:

$$
\underline{\boldsymbol{\sigma}}=\underline{\underline{\boldsymbol{\Lambda}}}: \underline{\boldsymbol{\varepsilon}}^{e} \quad(16) \quad \underline{\boldsymbol{M}}=\pi_{0} \lambda^{2} \operatorname{curl}\left(\underline{\boldsymbol{H}}^{p}\right)
$$

Combining the balance equation eq. (9) and eqs. (17) and (18) yields the expression of the micro-stress tensor:

$$
\underline{\boldsymbol{s}}=-\pi_{0} \lambda^{2} \operatorname{curl}\left(\operatorname{curl}\left(\underline{\boldsymbol{H}}^{p}\right)\right)=-\pi_{0} \lambda^{2} \epsilon_{j m s} \epsilon_{m l r} H_{i l, r s}^{p} \boldsymbol{e}_{i} \otimes \boldsymbol{e}_{j}
$$

The residual dissipation $D$ is then expressed as:

$$
D=\sum_{s=1}^{N_{s}}\left[\left(\tau^{s}-\chi^{s}\right) \dot{\gamma}^{s}-\tau_{c}^{s} \dot{\gamma}_{c u m}^{s}\right] \geq 0
$$

where are introduced, for the slip system $s$, the resolved shear stress (RSS) $\tau^{s}$, the backstress $\chi^{s}$ induced by the accumulation of GND, and $\tau_{c}^{s}$ the critical shear stress which is the thermodynamic force associated with $\gamma_{\text {cum }}^{s}$ :

$$
\tau^{s}=\underline{\boldsymbol{\sigma}}: \underline{\boldsymbol{\mu}}^{s} \quad(20) \quad \chi^{s}=-\underline{\boldsymbol{s}}: \underline{\boldsymbol{\mu}}^{s} \quad(21) \quad \tau_{c}^{s}=\rho \frac{\partial \psi_{s}}{\partial \gamma_{\text {cum }}^{s}}
$$

Then, the dissipation potentials $\boldsymbol{\Omega}^{s}\left(\tau^{s}-\chi^{s}, \tau_{c}^{s}\right)$ are introduced, defining the flow rule and the evolution equation of $\gamma_{\text {cum }}^{s}$ for each slip system:

$$
\dot{\gamma}^{s}=\frac{\partial \boldsymbol{\Omega}^{s}}{\partial\left(\tau^{s}-\chi^{s}\right)} \quad \dot{\gamma}_{c u m}^{s}=-\frac{\partial \boldsymbol{\Omega}^{s}}{\partial \tau_{c}^{s}}
$$


Each dissipation potential is assumed to depend on the Schmid yield function: $f^{s}\left(\tau^{s}-\chi^{s}, \tau_{c}^{s}\right)=\left|\tau^{s}-\chi^{s}\right|-\tau_{c}^{s}$ such that $\mathbf{\Omega}^{s}=\mathbf{\Omega}^{s}\left(f^{s}\left(\tau^{s}-\chi^{s}, \tau_{c}^{s}\right)\right)$, and that eq. (19) is satisfied under the condition $\frac{\partial \mathbf{\Omega}^{s}}{\partial f^{s}}>0$. A viscoplastic power law potential compatible with these restrictions is chosen and yields the classical Norton flow rule:

$$
\mathbf{\Omega}^{s}=\frac{K}{n+1}\left\langle\frac{\left|\tau^{s}-\chi^{s}\right|-\tau_{c}^{s}}{K}\right\rangle^{n+1} \Rightarrow \dot{\gamma}^{s}=\operatorname{sign}\left(\tau^{s}-\chi^{s}\right)\left\langle\frac{\left|\tau^{s}-\chi^{s}\right|-\tau_{c}^{s}}{K}\right\rangle^{n}
$$

where $\langle x\rangle$ denotes the positive part of $x$, and $n$ and $K$ are respectively the Norton law exponent and coefficient. The model is finally completed with a simple and generic equation governing the evolution of the critical resolved shear stress:

$$
\tau_{c}^{s}=\tau_{0}^{s}-\Delta \tau^{s}\left[1-\exp \left(-\frac{\gamma_{c u m}^{s}}{\gamma_{0}}\right)\right] \text { with } \quad \dot{\gamma}_{c u m}^{s}=\left|\dot{\gamma}^{s}\right|
$$

Equation (25) induces a material softening that will trigger slip localization, and involves three material parameters: $\tau_{0}^{s}, \Delta \tau^{s}$ and $\gamma_{0}$ that are respectively the initial critical resolved shear stress, the maximum softening, and a parameter adjusting the softening rate. The softening ratio $\eta=\frac{\Delta \tau}{\tau_{0}}$ is used to further to interpret the numerical simulations $(0 \leq \eta \leq 1)$.

In addition, please note that if a specific length unit $L$ is chosen, eq. (18) can classically be written with a dimensionless spatial derivative (denoted by the subscript $A_{, x^{\prime}}$ ):

$$
\underline{\boldsymbol{s}}=-\pi_{0} \frac{\lambda^{2}}{L^{2}} \epsilon_{j m s} \epsilon_{m l r} H_{i l, r^{\prime} s^{\prime}}^{p} \boldsymbol{e}_{i} \otimes \boldsymbol{e}_{j}
$$

The results thus depend on the ratio $\frac{\lambda^{2}}{L^{2}}$, which will be used to quantify the intensity of non-local effects in the presented applications. The length unit will be the characteristic length of the crystalline unit cell or the mean grain size $d_{g}$ for polycrystalline unit cells.

\subsection{Application to the modeling of ideal slip and kink bands}

This curl $\left(H^{p}\right)$ theory is very often employed to study size effects in the hardening behavior of metals. In the present work, it is applied to the modeling of individual SBs/KBs formation. To this end, a set of simplifying hypotheses are assumed, and detailed hereafter. A SB or KB is considered as a localization band comprised between two planar boundaries, consisting in a crystal deforming through single slip (therefore the subscript $s$ is dropped for this section), comprised between two elastic layers. The layers are perpendicular to $\boldsymbol{n}$ for a SB and perpendicular to $\boldsymbol{m}$ for a KB. Besides, the bands are studied here far from their in-plane edges and hence, they are considered as homogeneous with respect to their in-plane coordinates. Finally, the following kinematic hypothesis is assumed: the localization band undergoes a pure shear loading of mean value $\bar{\gamma}$, imposed by the motion of the elastic layers (whose deformation is neglected). This approximation is schematized in fig. 2 .

For the sake of simplicity, it is assumed that the material has a linear isotropic elastic behavior (eq. (27)), characterized by Lamé coefficients $\lambda$ and $\mu$, and, instead of the viscoplastic behavior described by eqs. (24) and (25), a linear hardening rate-independent plastic behavior (eq. (28)), characterized by the hardening modulus $H$ and the initial critical resolved shear stress (CRSS) $\tau_{0}$, such that:

$$
\begin{aligned}
\underline{\boldsymbol{\sigma}} & =\lambda \operatorname{tr}\left(\underline{\boldsymbol{\varepsilon}}^{e}\right) \underline{\mathbf{1}}+2 \mu \underline{\boldsymbol{\varepsilon}}^{e} \\
\tau_{c} & =\tau_{0}+H \gamma_{\text {cum }}
\end{aligned}
$$

\section{Slip band case}

Considering the modeled geometry and loading (fig. $2(\mathrm{a})$ ), the problem is $x_{3}$ and $x_{m}$ invariant. Thus, denoting by $\boldsymbol{u}$ the displacement:

$$
\boldsymbol{u}=u\left(x_{n}\right) \boldsymbol{m} \Rightarrow \nabla \underline{\boldsymbol{u}}=u_{, n} \boldsymbol{m} \otimes \boldsymbol{n}
$$




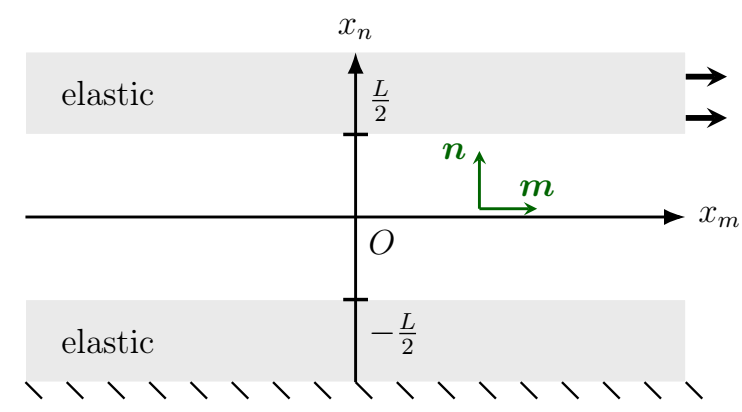

(a) slip band

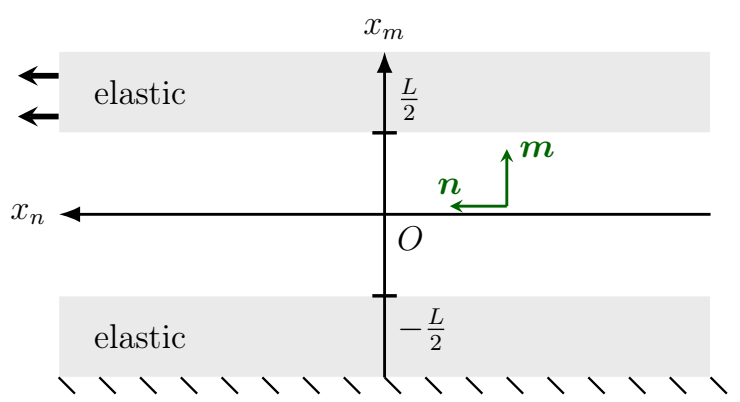

(b) kink band

Figure 2: Ideal modeling of the local shearing of a crystal within a slip localization band for a slip band (a) and a kink band (b). The central phase is elasto-plastic and deforms through a singe slip system, whose orientation is indicated in red. The considered geometry is invariant with respect to the $x_{m}$ coordinate in (a), and with respect to $x_{n}$ in (b).

Injecting eq. (29) and eq. (1) in eq. (27) yields:

$$
\underline{\boldsymbol{H}}^{e}=\left(u_{, n}-\gamma\right) \boldsymbol{m} \otimes \boldsymbol{n}(30) \quad \underline{\boldsymbol{\omega}}_{e}=\frac{1}{2}\left(u_{, n}-\gamma\right)(\boldsymbol{m} \otimes \boldsymbol{n}-\boldsymbol{n} \otimes \boldsymbol{m})(31) \quad \underline{\boldsymbol{\sigma}}=\mu\left(u_{, n}-\gamma\right)(\boldsymbol{m} \otimes \boldsymbol{n}+\boldsymbol{n} \otimes \boldsymbol{m})
$$

According to eqs. (2), (6) and (18), the expression of $\underline{\boldsymbol{\alpha}}$ and $\chi$ written in the basis $\left\{\boldsymbol{m}, \boldsymbol{n}, \boldsymbol{e}_{3}=\boldsymbol{m} \times \boldsymbol{n}\right\}$ are:

$$
\begin{aligned}
& \underline{\boldsymbol{\alpha}}=-\left[\gamma_{, 3} \boldsymbol{m} \otimes \boldsymbol{m}-\gamma_{, m} \boldsymbol{m} \otimes \boldsymbol{e}_{3}\right] \\
& \chi=-\lambda^{2} \pi_{0}(\gamma, m m+\gamma, 33)
\end{aligned}
$$

From the $x_{3}$ and $x_{m}$ invariance follows that $\underline{\boldsymbol{\alpha}}=0$ and $\chi=0$, which implies that SBs do not involve GND accumulation nor backstress, far from their in-plane edges. This model is thus equivalent to a CCP for SBs. Combining eqs. (8) and (32) gives $\sigma_{m n, n}=\tau_{, n}=0$. Hence, the yield condition $|\tau|=\tau_{0}$ is verified everywhere in the band and after eq. (28), for a monotonic evolution of the slip variable we have:

$$
\mu\left(u_{, n}-\gamma\right)=\tau_{0}+H \gamma
$$

In the case $H>0$, by taking the mean of eq. (35), it is easy to verify that homogeneous deformation and plastic slip fields are solution of the equation, with $\gamma=\left(\bar{\gamma}-\frac{\tau_{0}}{\mu}\right) /\left(1+\frac{H}{\mu}\right)$. In the case $H \leq 0$, according to Asaro and Rice (1977) bifurcation analysis, plastic instability occurs at the yield point and plastic slip localizes into a band of undetermined width. Moreover, note that under the assumptions (reasonable for a large variety of crystals) of negligible elastic strain $\left(\mu \gg \tau_{0}\right)$, of moderate hardening $(\mu \gg|H|)$, and considering incipient plasticity localization $(\gamma \ll 1)$, eqs. (5), (31) and (35) give $\left(u_{, n}-\gamma\right) \ll 1 \Rightarrow \theta=0$, which implies that no lattice rotation is produced when slip bands form (far from their in-plane edges).

Kink band case

Considering the modeled geometry (fig. 2 (a)), the problem is $x_{3}$ and $x_{n}$ invariant, so that:

$$
\boldsymbol{u}=u\left(x_{m}\right) \boldsymbol{n} \Rightarrow \nabla \underline{\boldsymbol{u}}=u_{, m} \boldsymbol{n} \otimes \boldsymbol{m}
$$

Injecting eq. (29) and eq. (1) in eq. (27) yields:

$$
\underline{\boldsymbol{H}}^{e}=u,{ }_{m} \boldsymbol{n} \otimes \boldsymbol{m}-\gamma \boldsymbol{m} \otimes \boldsymbol{n} \quad(37) \quad \underline{\boldsymbol{\omega}}_{e}=\underline{\boldsymbol{H}}^{e}(38) \quad \underline{\boldsymbol{\sigma}}=\mu\left(u,{ }_{, m}-\gamma\right)(\boldsymbol{m} \otimes \boldsymbol{n}+\boldsymbol{n} \otimes \boldsymbol{m})
$$

where eq. (1) was used to derive eq. (37). After eqs. (33) and (34):

$$
\begin{aligned}
& \underline{\boldsymbol{\alpha}}=\gamma_{, m} \boldsymbol{m} \otimes \boldsymbol{e}_{3} \\
& \chi=-\lambda^{2} \pi_{0} \gamma_{, m m}
\end{aligned}
$$


showing that KB formation involves production of GND and the subsequent backstress. Then, after substituting the value of $\chi$ in the Schmid criterion and some manipulations, one obtains the differential equation governing the slip variable:

$$
\gamma_{, m m}-\frac{1}{\lambda_{0}^{2}} \gamma+\frac{1}{\lambda_{0}^{2}} \frac{\tau-\tau_{0}}{H}=0
$$

with $\lambda_{0}=\lambda \sqrt{\frac{\pi_{0}}{H}}$. Additional boundary condition for the plastic slip must also be considered for the problem to be well-posed. It has been shown by Cordero et al. (2010) that with the $\operatorname{curl}\left(\underline{\boldsymbol{H}}^{p}\right) \operatorname{model}, \underline{\boldsymbol{m}}$ from eq. (11) must exhibit a jump at plastic-elastic interfaces in order to allow for non zero slip in the plastic phase. Thus, no condition is enforced at the boundary for $\underline{\boldsymbol{m}}$. Instead, it is assumed that the solution slip field must verify the problem symmetry, and vanish at the $\mathrm{KB}$ edges, which reduce to the following conditions:

$$
\text { Continuity: } \gamma\left( \pm \frac{L}{2}\right)=0
$$

Symmetry: $\gamma\left(x_{m}\right)=\gamma\left(-x_{m}\right)$

Finally, according to the balance equation eq. (8): $\sigma_{m n, m}=0$, hence $\tau$ is constant over the localization band. Thus it is equal to its mean over the whole localization band, i.e the mean imposed shear $\bar{\gamma}$. This condition provide an equation to compute $\tau$, which writes:

$$
\tau=\frac{1}{L} \int_{\frac{-L}{2}}^{\frac{L}{2}} \tau d x_{m}=\frac{\mu}{L} \overline{u_{, n}}-\frac{\mu}{L} \int_{\frac{-L}{2}}^{\frac{L}{2}} \gamma\left(x_{m}\right) d x_{m}=\mu \bar{\gamma}-\frac{\mu}{L} \int_{\frac{-L}{2}}^{\frac{L}{2}} \gamma\left(x_{m}\right) d x_{m}
$$

The solution of eq. (42) depends on the value of $H$, and the three cases $H>0, H=0$ and $H<0$ must be reviewed ${ }^{2}$.

Case $H>0$ :

The solution takes the form $\gamma=\alpha \cosh \left(\frac{x_{m}}{\lambda_{0}}\right)+\beta \sinh \left(\frac{x_{m}}{\lambda_{0}}\right)+\frac{\tau-\tau_{0}}{H}$. Using the continuity and symmetry condition, and eq. (43) yields:

$$
\gamma\left(x_{m}\right)=\frac{\tau-\tau_{0}}{H}\left[1-\frac{\cosh \left(\frac{x_{m}}{\lambda_{0}}\right)}{\cosh \left(\frac{L}{2 \lambda_{0}}\right)}\right] \quad \text { with } \tau=\frac{\bar{\gamma}-\frac{\tau_{0}}{K_{1}}}{\frac{1}{\mu}-\frac{1}{K_{1}}} \quad \text { and } K_{1}=\frac{H}{\frac{2 \lambda_{0}}{L} \tanh \left(\frac{L}{2 \lambda_{0}}\right)-1}
$$

Case $H=0$ :

In this case the solution takes a parabolic form whose general solution is $\gamma=\left(\frac{\tau_{0}-\tau}{2 \lambda^{2} \pi_{0}}\right) x_{m}^{2}+\alpha x_{m}+\beta$. Using the continuity and symmetry condition, and eq. (43) yields:

$$
\gamma\left(x_{m}\right)=\left(\frac{\tau-\tau_{0}}{2 \lambda^{2} \pi_{0}}\right)\left[\left(\frac{L}{2}\right)^{2}-x_{m}^{2}\right] \quad \text { with } \tau=\frac{\bar{\gamma}+\frac{\tau_{0}}{K_{2}}}{\frac{1}{\mu}-+\frac{1}{K_{2}}} \quad \text { and } K_{2}=\frac{12 \lambda^{2} \pi_{0}}{L^{2}}
$$

Case $H<0$ :

In this case eq. (42) is written under the form:

$$
\gamma_{, m m}+\frac{2 \pi^{2}}{\lambda_{0}^{\prime}} \gamma+\frac{2 \pi^{2}}{\lambda_{0}^{\prime}} \frac{\tau-\tau_{0}}{|H|}=0 \quad \text { where } \lambda_{0}^{\prime}=2 \pi \lambda \sqrt{\frac{\pi_{0}}{|H|}}
$$

This equation admits general solutions of the form $\gamma=\alpha \cos \left(\frac{2 \pi x_{m}}{\lambda_{0}^{\prime}}\right)+\beta \sin \left(\frac{2 \pi x_{m}}{\lambda_{0}^{\prime}}\right)+\frac{\tau-\tau_{0}}{H}$. As $H<0$, plastic instability and localization should occur at yield. Hence, a solution where the plastic slip is non zero in a localized band of width $\omega_{b}$ is sought, which modifies the continuity condition. In addition, the boundaries of the band are inside the plastic phase, thus the condition of vanishing generalized traction can be applied:

\footnotetext{
${ }^{2}$ It must be noted that these calculations are analogous to those presented in Scherer et al. (2019), where a micromorphic model based on the gradient of a cumulated slip variable is used to model a shear band.
} 
Continuity: $\gamma\left( \pm \frac{\omega_{b}}{2}\right)=0$

Microfree edges: $\underline{\boldsymbol{m}}\left( \pm \frac{\omega_{b}}{2}\right)=\underline{\boldsymbol{M}}\left( \pm \frac{\omega_{b}}{2}\right) \cdot \underline{\boldsymbol{\epsilon}} \cdot \boldsymbol{n}=0 \quad \Longleftrightarrow \quad \gamma_{, n}\left( \pm \frac{\omega_{b}}{2}\right)=0$

These conditions, along with eq. (43) yield:

$$
\begin{aligned}
\gamma\left(x_{m}\right) & =\frac{\tau-\tau_{0}}{H}\left[1+\cos \left(\frac{2 \pi x_{m}}{\lambda_{0}^{\prime}}\right)\right] \quad \text { with } \tau=\frac{\bar{\gamma}+\frac{\tau_{0}}{K_{3}}}{\frac{1}{\mu}+\frac{1}{K_{3}}} \quad \text { and } K_{3}=\frac{L H}{\lambda_{0}^{\prime}} \\
\omega_{b} & =\lambda_{0}^{\prime}=2 \pi \lambda \sqrt{\frac{\pi_{0}}{|H|}}
\end{aligned}
$$

To estimate the lattice rotation angle within $\mathrm{KB}$, the yield condition is used, which writes:

$$
\mu\left(u_{, n}-\gamma\right)=\tau_{0}+H \gamma-\lambda^{2} \pi_{0} \gamma_{, m m}
$$

Like in the SB case, it is assumed that all plasticity related shear stress terms in eq. (49) on the right hand-side are negligible with respect to the elastic shear modulus $\mu$ and that $\gamma \ll 1$ (i.e. elastic deformation and hardening effects are neglected at incipient plasticity), thus $\left(u_{, n}-\gamma\right) \approx 0$. Acknowledging that the lattice rotation axis is orthogonal to the $(\boldsymbol{m}, \boldsymbol{n})$ plane, the lattice rotation angle $\theta$ can be approximated using eqs. (5), (37) and (38):

$$
\underline{\omega}_{e}=\gamma(\boldsymbol{n} \otimes \boldsymbol{m}-\boldsymbol{m} \otimes \boldsymbol{n}) \approx \theta(\boldsymbol{n} \otimes \boldsymbol{m}-\boldsymbol{m} \otimes \boldsymbol{n}) \Rightarrow \theta \approx \gamma
$$

Finally, the dislocation density tensor field in the KB is given by:

$$
\underline{\boldsymbol{\alpha}}=\frac{2 \pi\left(\tau_{0}-\tau\right)}{\lambda_{0}^{\prime} H} \sin \left(\frac{2 \pi x_{m}}{\lambda_{0}^{\prime}}\right) \boldsymbol{m} \otimes \boldsymbol{e}_{3}
$$

Overall, this analytic study of individual SB/KB demonstrates that SGP models based on Nye's tensor only affect $\mathrm{KB}$ modeling when compared to $\mathrm{CCP}$ models. Moreover, it provides an analytic solution to validate numerical implementations of the theory. It also shows, in the context of softening SGP, that KB should be regularized and form with a finite width $\omega_{b}$ which is proportional to the material length scale $\lambda$. Besides, eq. (51) predicts the formation of two GND density maxima, located at $\pm \omega_{b} / 2$ and of opposite signs. They coincide with the boundaries of the localization band. Hence, the theory also predicts the formation of the GND walls at the boundaries of a KB.

\section{Numerical implementation in a FFT-based solver}

\subsection{Algorithm}

The SGP model presented in section 2.1 is solved numerically using a spectral homogenization scheme, based on the now well-established Fast Fourier Transform (FFT) method. Simulations are performed using the in-house FFT solver AMITEX_FFTP ${ }^{3}$. First, its algorithm is birefly presented in the context of classical FFT-based methods, and details are provided about how the additional equations from the SGP model are integrated within this framework.

A periodic unit cell discretized by a regular grid of $N$ voxels $\left(N=N_{1} \times N_{2} \times N_{3}\right)$ is considered, and submitted to a prescribed mean deformation state $\underline{\boldsymbol{E}}$, over which the various mechanical fields are assigned a voxel-wise constant value. $\boldsymbol{x}_{i}=\boldsymbol{x}\left(i_{1}, i_{2}, i_{3}\right)=\left(x_{i_{1}}, x_{i_{2}}, x_{i_{3}}\right)$ and $\boldsymbol{\xi}_{i}=\boldsymbol{\xi}\left(k_{1}, k_{2}, k_{3}\right)=\left(\xi_{k_{1}}, \xi_{k_{2}}, \xi_{k_{3}}\right)$ denote the grid points coordinates respectively in real and in Fourier space. To solve the mechanical problem consisting in the Cauchy stress balance (eq. (8)), the constitutive equations and the periodic boundary conditions, the

${ }^{3}$ http://www . maisondelasimulation.fr/projects/amitex/html/ 
classical basic scheme of Moulinec and Suquet (1998) is used. It consists in solving iteratively the following equations until mechanical equilibrium is reached:

$$
\begin{aligned}
\underline{\boldsymbol{\tau}}\left(\boldsymbol{x}_{i}\right) & =\underline{\boldsymbol{\sigma}}\left(\boldsymbol{x}_{i}\right)-\underline{\underline{\boldsymbol{\Lambda}}}^{0}: \frac{1}{2}\left(\nabla \underline{\boldsymbol{u}}\left(\boldsymbol{x}_{i}\right)+\nabla \underline{\boldsymbol{u}}^{T}\left(\boldsymbol{x}_{i}\right)\right) \quad \forall \boldsymbol{x}_{i} \in \Omega \\
\widehat{\nabla \underline{\boldsymbol{u}}}\left(\boldsymbol{\xi}_{i}\right) & =\underline{\underline{\hat{\boldsymbol{\Gamma}}}}^{0}: \underline{\hat{\boldsymbol{\tau}}} \quad \forall \boldsymbol{\xi}_{i} \neq 0 \underline{\hat{\boldsymbol{\varepsilon}}}(0)=\underline{\boldsymbol{E}}
\end{aligned}
$$

where $\underline{\boldsymbol{\tau}}, \underline{\boldsymbol{\sigma}}$ and $\underline{\boldsymbol{\varepsilon}}$ are respectively the polarisation, Cauchy stress and strain tensors in real space, $\underline{\hat{\boldsymbol{\varepsilon}}}$ and $\underline{\hat{\boldsymbol{\tau}}}$ are the strain and polarisation tensors in Fourier space. $\underline{\underline{\Lambda}}^{0}$ is the elastic stiffness tensor of a reference isotropic linear elastic material.

Note that our formulation relies on $\nabla \underline{\boldsymbol{u}}$ as integration variable instead of the strain tensor $\underline{\boldsymbol{\varepsilon}}$ used for the classical implementation of this algorithm. This difference is central to the implementation of the present theory as in order to properly model the differences between SBs and KBs, dependence on the non symmetric displacement gradient $\nabla \underline{\boldsymbol{u}}$ and its plastic part $\underline{\boldsymbol{H}}^{p}$ is mandatory.

In our implementation, the fixed-point integration is enhanced by Anderson (1965) convergence acceleration technique. This algorithm drastically improves the convergence of the basic scheme. Besides, for non-linear materials simulation, compared to a Newton-Raphson implementation (Gélébart and MondonCancel, 2013), it does not require the tedious evaluation of the tangent behavior. The reader is referred to Chen et al. (2019) for a detailed description of its implementation in the solver. Moreover, instead of the original truncated Green operator proposed by Moulinec and Suquet (1998), $\underline{\underline{\boldsymbol{\Gamma}}}^{0}$ refers here to the modified discrete operator proposed by Willot (2015a) that is implemented in AMITEX_FFTP. Schneider et al. (2017) have demonstrated the equivalence between the use of this operator and to solve the same mechanical problem with cubic linear finite elements with reduced integration. In the present work, this operator is not symmetrized in order to comply with the $\nabla \underline{\boldsymbol{u}}$ based formulation (the reader is referred to Moulinec and Suquet (1998); Willot (2015a) for more details on the Green operator computation). Finally, at each time step, the constitutive equations eqs. (1), (2), (16), (24) and (25) are integrated using a fully implicit $\theta$-method implemented in a UMAT subroutine, through to the MFront code generator (Helfer et al., 2015). Additionally, note that the computation of eqs. (52) and (53) are implemented in a massively parallel fashion, which allows to make the most of powerful computing facilities and address the simulation of large unit cells.

In the SGP theory, it appears that the only non-local quantities required to solve the problem are the backstresses $\chi^{s}$, that intervene in eq. (24). Their computation requires the evaluation of $\underline{s}$ through eq. (18). Following the FFT-based implementation of a similar model based on Gurtin's theory proposed by Lebensohn and Needleman (2016), the whole problem is solved by coupling our fixed-point algorithm to an explicit update of the backstresses $\chi^{s}$ through the computation of eq. (18) in Fourier space, at each time step, after reaching mechanical equilibrium. Hence, this implementation requires to prescribe sufficiently small loading increments in order to avoid a numerical instability of the algorithm. The complete algorithm is summarized in fig. 3 .

\subsection{Backstress calculation in Fourier space}

The crucial step in the implementation of the non-local theory is the computation of the microstress tensor in eq. (18). The accurate computation of the partial derivatives terms $H_{i l, r s}^{p}$ is the critical part of its evaluation. Several studies have now established that using a differentiation scheme based on finite-differences (FD), instead of using continuous Fourier transforms (CFT) derivation formula prior to discretization, results in an improved smoothness of computed derivative fields (Berbenni et al., 2014; Willot, 2015a). This strongly reduces the apparition of spurious oscillations in the simulated fields, which increases FFT-based solvers accuracy and convergence rate. For this reason, Lebensohn and Needleman (2016) have used a 27-voxel centered second order FD scheme, proposed by Berbenni et al. (2014) to compute the backstress in their FFT-based implementation of Gurtin's theory. They demonstrated that this scheme satisfacto- 


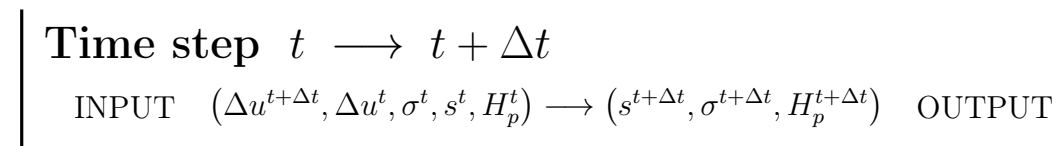

\section{Initial guess}

$$
\Delta u^{0}=\Delta u^{t}+\frac{\Delta_{t}}{\Delta_{t-1}}\left(\Delta u^{t}-\Delta u^{t-\Delta t}\right) \quad\left(\sigma^{0}, H_{p}^{0}\right)=\mathcal{F}\left(\Delta u^{0}, \Delta u^{t}, \sigma^{t}, s^{t}, H_{p}^{t}, \Delta t\right)
$$

II Fix-point Algorithm : iteration $k$

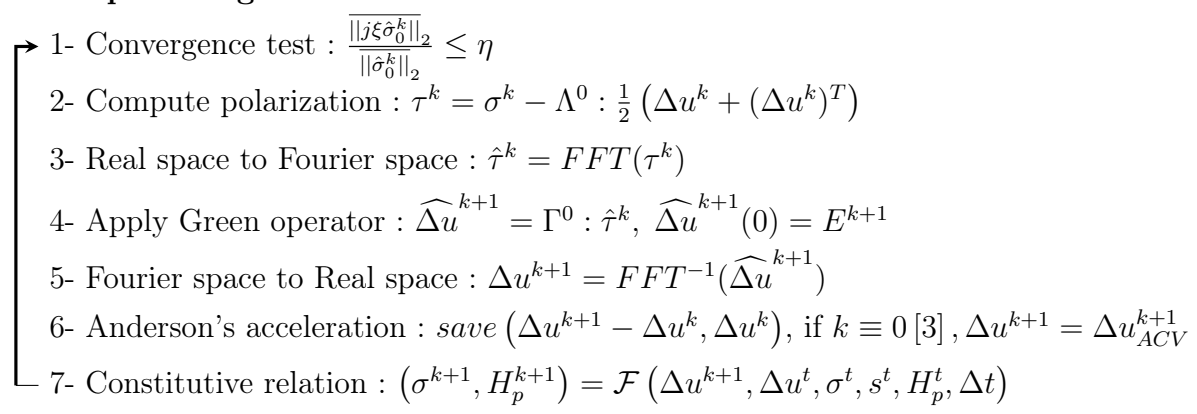

III Solve microstress equation

$$
s_{i j}^{t+\Delta t}=-\pi_{0} \lambda^{2} \epsilon_{j m s} \epsilon_{m l r} F F T^{-1}\left(\widehat{H}_{i l, r s}^{p, t+\Delta t}\right)
$$

Figure 3: Algorithm implemented to solve the SGP plasticity model. The constitutive relations are symbolically represented by the operator $\mathcal{F}$. For readability, its dependence on the various internal variables and material coefficients has been omitted, except for the plastic part of the displacement gradient, which is written here exceptionally with the index $p$ instead of superscript $\left(H_{p}\right.$ instead of $\left.H^{p}\right)$ in order to avoid conflict with the subscript notation indicating the time increment $(t$ or $\Delta t)$ or the iteration counter $k$.

rily evaluates the backstresses sharp variations close to grain boundaries, resulting in a sound numerical implementation of the theory ${ }^{4}$

However, the aim of Lebensohn and Needleman (2016) was to propose a FFT-based framework to simulate grain-size effects in polycrystals. In the present work, we want to raise the concern on another numerical issue associated to the differentiation scheme used to implement the non-local theory. Indeed, as shown by the analytic study of section 2.2, the other physically significant property of SGP models based on Gurtin's theory is their distinct impact on SB and KB. Hence, a numerical implementation of this theory aimed at slip localization simulation, needs to ensure that the differentiation scheme used to compute eq. (18) actually yields $\underline{\boldsymbol{s}}=0$ in SBs, and $\underline{\boldsymbol{s}} \neq 0$ in KBs. To investigate this left out issue, three different numerical schemes to compute the double curl operator have been tested.

When using a FD based scheme, differentiation in Fourier space can still be expressed as a product between the function to which the derivation operator is applied, and a complex number called a modified frequency (by contrast to the usual frequencies $\boldsymbol{\xi}=\left(\xi_{1}, \xi_{2}, \xi_{3}\right)$ involved in classical CFT differentiation formulas). This writes:

$$
\begin{array}{lll}
\partial_{i}^{*}(\hat{f}(\boldsymbol{\xi}))=\boldsymbol{j} \xi_{i}^{*} \hat{f}(\boldsymbol{\xi}) & \partial_{i i}^{*}(\hat{f}(\boldsymbol{\xi}))=\xi_{i i}^{*} \hat{f}(\boldsymbol{\xi}) & \partial_{i j}^{*}(\hat{f}(\boldsymbol{\xi}))=\xi_{i j}^{*} \hat{f}(\boldsymbol{\xi}) \\
\partial_{i}^{\circ}(\hat{f}(\boldsymbol{\xi}))=\boldsymbol{j} \xi_{i}^{\circ} \hat{f}(\boldsymbol{\xi}) & \partial_{i i}^{\circ}(\hat{f}(\boldsymbol{\xi}))=-\xi_{i}^{\circ} \xi_{i}^{\circ} \hat{f}(\boldsymbol{\xi}) & \partial_{i j}^{\circ}(\hat{f}(\boldsymbol{\xi}))=-\xi_{i}^{\circ} \xi_{j}^{\circ} \hat{f}(\boldsymbol{\xi})
\end{array}
$$

\footnotetext{
${ }^{4}$ Note additionnaly that Willot's modified Green operator (Willot, 2015b) is based on such a FD operator, usually called the "rotated" FD scheme.
} 
In eqs. (54) and (55), $\boldsymbol{j}$ is the complex unit, $\partial_{i}$ and $\partial_{i j}$ denote resp. the first/second order derivation operator with respect to coordinate $i / i$ and $j$, and the superscripts $*$ and $\circ$ denote resp. the derivation operators based on the previously mentioned 27-voxels centered FD resp. rotated FD schemes. In order to alleviate the presentation, the expressions of the modified frequencies are not recalled here, for more details on their derivation the reader is referred to Lebensohn and Needleman (2016); Berbenni et al. (2014); Willot (2015a). From these derivation operators, the following tensorial differentiation operators are constructed:

$$
\begin{gathered}
\operatorname{curl}^{*}\left(\underline{\hat{\boldsymbol{H}}}^{p}\right)=\epsilon_{j m s} \partial_{s}^{*}\left(\hat{H}_{i m}^{p}\right) \boldsymbol{e}_{i} \otimes \boldsymbol{e}_{j} \\
\operatorname{curlcurl}^{*}\left(\underline{\hat{\boldsymbol{H}}}^{p}\right)=\epsilon_{j m s} \epsilon_{m l r} \partial_{r s}^{*}\left(\hat{H}_{i l}^{p}\right) \boldsymbol{e}_{i} \otimes \boldsymbol{e}_{j} \\
\operatorname{curlcurl}^{\circ}\left(\underline{\hat{\boldsymbol{H}}}^{p}\right)=\epsilon_{j m s} \epsilon_{m l r} \partial_{r s}^{\circ}\left(\hat{H}_{i l}^{p}\right) \boldsymbol{e}_{i} \otimes \boldsymbol{e}_{j}
\end{gathered}
$$

An analytic two-dimensional analysis of the backstress calculation using the underlying FD formulation of the operators in eq. (54), on simplified geometrical configurations of localization bands has been conducted in Appendix A . It reveals that the operators curl ${ }^{*}$ and curlcurl ${ }^{*}$ can only preserve exactly the property $\{\underline{\boldsymbol{\alpha}}=0$ and $\underline{\boldsymbol{s}}=0$ in SBs $\}$ in some specific cases when the localization band normal is aligned with the grid directions. Otherwise, using them leads to a non-vanishing backstress in SBs, that is of the same order of magnitude as the backstress arising in KBs. However, the study also evidences that when computing the backstresses using twice in a row the curl* operator, the computed backstress in the SB becomes significantly lower than the computed backstress in the KB. This led us to introduce the following third double curl operator:

$$
\operatorname{curl} 2^{*}\left(\underline{\hat{\boldsymbol{H}}}^{p}\right)=\epsilon_{j m s} \partial_{s}^{*}\left(\epsilon_{m l r} \partial_{r}^{*}\left(\hat{H}_{i l}^{p}\right)\right) \boldsymbol{e}_{i} \otimes \boldsymbol{e}_{j}
$$

The study has not been carried out for the curlcurl ${ }^{\circ}$ operator because its underlying 2nd order derivative operator already is consistent with a successive application of two first order derivatives (see eq. (55), $\operatorname{curl} 2^{\circ}=\operatorname{curlcurl}^{\circ}$ ).

Figure 4 shows a numerical calculation carried out with these operators on a periodic two dimensional test $\underline{\boldsymbol{H}}^{p}$ field, represented in figure (a), consisting in two crossing localization bands: a SB and a KB. One component of the double curl of this field computed with resp. curl $2^{*}$, curlcurl ${ }^{*}$ and curlcurl $^{\circ}$ is displayed resp. in figure (b), (c) and (d). Accordingly with the analytic study of Appendix A, it appears that the operator curl $2^{*}$ is the best suited to preserve the property $\{\underline{\boldsymbol{\alpha}}=0$ and $\underline{\boldsymbol{s}}=0$ in SBs $\}$ for a band of random orientation. On the contrary, the operator curlcurl* used in Lebensohn's and Needleman's implementation of Gurtin's theory, appears clearly ill-suited to preserve it. Numerical simulations presented in section 4.1 will confirm the crucial influence of these errors on the development of SB and KB in numerical simulations.

All these considerations led us to choose operator curl2* to compute the micro-stress tensor. Finally, its computation is implemented as follows:

$$
\underline{\boldsymbol{s}}=-\pi_{0} \lambda^{2} F F T^{-1}\left\{\epsilon_{j m s} \partial_{s}^{*}\left(\epsilon_{m l r} \partial_{r}^{*}\left(\hat{H}_{i l}^{p}\right)\right) \boldsymbol{e}_{i} \otimes \boldsymbol{e}_{j}\right\}
$$

The involved evaluation of the differentiation operators $\partial^{*}$ is implemented in a massively parallel fashion, allowing the efficient simulation of this SGP model on large unit cells, with parallel computers.

Regarding the backstresses calculation, one last point is worth emphasizing. Lebensohn and Needleman (2016) used a model formulated with the gradient of each slip variable as non-local variables to implement their FFT implementation. This approach involves the derivatives of the Schmid tensors in the backstresses expressions. It requires the definition of corresponding slip systems in neighboring grains to evaluate derivates at grain boundaries, which is a cumbersome geometrical and bookkeeping issue. Besides, Lebensohn and Needleman (2016) showed that neglecting these terms leads to an underestimation of nonlocal effects that grows with grain to grain misorientation. With the $\operatorname{curl}\left(\underline{\boldsymbol{H}}^{p}\right)$ model, no Schmid tensor derivatives appear in the equations, preventing this issue. It is in fact a major numerical advantage of the $\operatorname{curl}\left(\underline{\boldsymbol{H}}^{p}\right)$ model for polycrystalline simulations. 


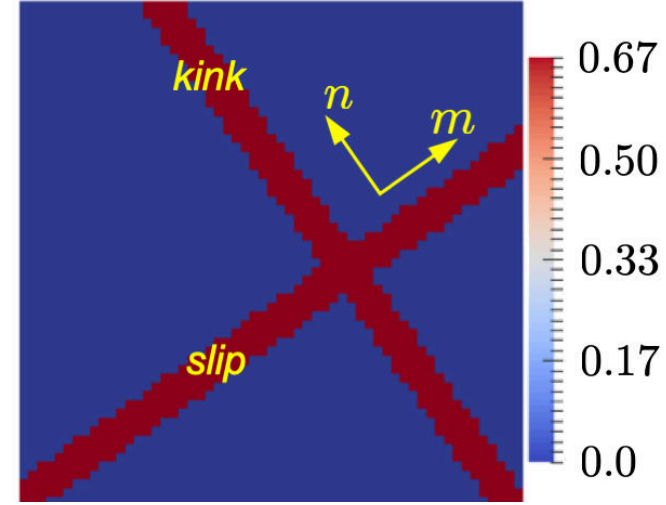

(a) $H_{23}^{p}$

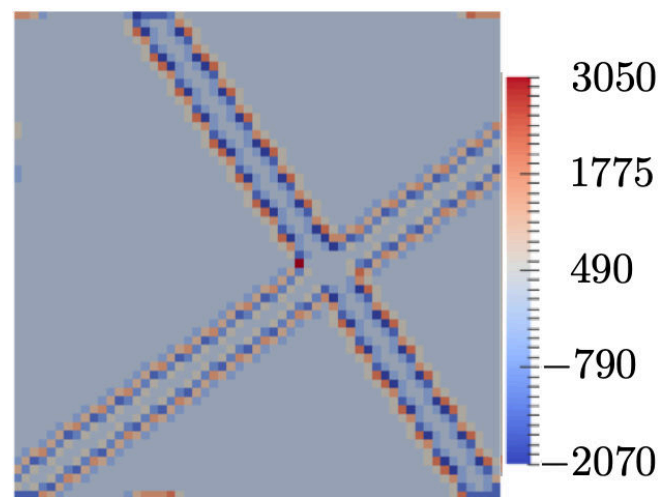

(c) $L^{2} \operatorname{curlcurl}^{*}\left(\underline{\boldsymbol{H}}^{p}\right)_{22}$

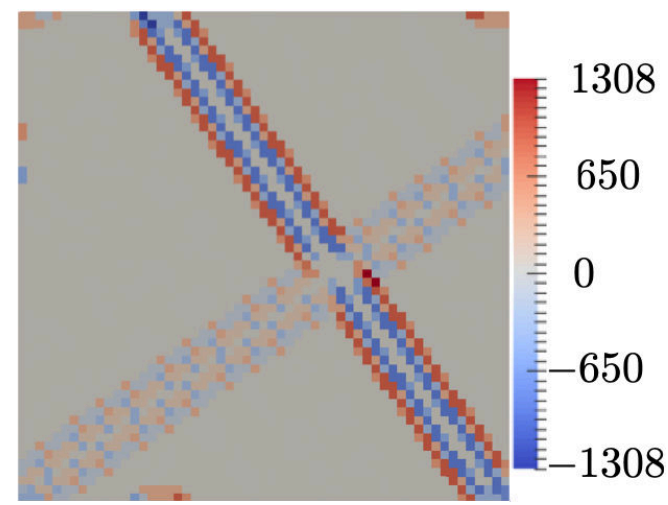

(b) $L^{2} \operatorname{curl} 2^{*}\left(\underline{\boldsymbol{H}}^{p}\right)_{22}$

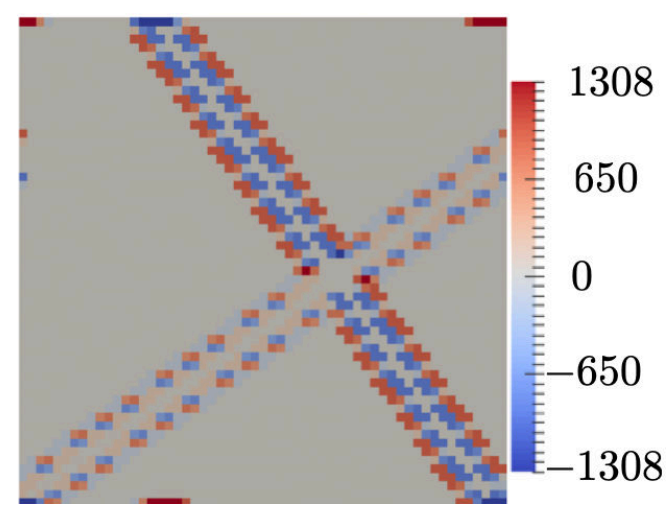

(d) $L^{2} \operatorname{curlcurl}^{\circ}\left(\underline{\boldsymbol{H}}^{p}\right)_{22}$

Figure 4: Computed double curl of the $\boldsymbol{H}^{p}$ field in (a) representing one slip and one kink band crossing with the operators curl $2^{*}$ (b), curlcurl* (c) and curlcurl ${ }^{\circ}$ (d), defined in section 3.2. The fields display the dimensionless quantity $L^{2} \operatorname{curlcurl}\left(H_{p}\right)$, where unit cell size $L$ is set to 1 in an arbitrary length unit.

\subsection{Practical imposition of interface conditions at grain boundaries}

The second numerical question associated with our non-local theory arises when considering the higher order boundary conditions associated to eq. (11), that have to be specified on grain boundaries for $\underline{\boldsymbol{m}}$ or $\underline{\boldsymbol{H}}^{p}$ when conducting polycrystalline simulations. Handling explicit interface conditions on the interior of the periodic unit-cell with spectral methods is a complex issue and still an open problem. Improved FFT methods based on composite voxels have recently been proposed to account for the proper location and orientation of interfaces in voxelized microstructures (Gélébart and Ouaki, 2015; Kabel et al., 2017; Marano and Gélébart, 2020). Their extension to non-local interface conditions is a promising but challenging problem, regarding the relevant definition of derivatives for each constituent phase of the composite voxels. Besides, these conditions would have to be imposed through a penalty method, which is not compatible with our current fixed-point implementation. As our main focus is on localization, simpler solutions to impose these conditions are investigated in the present work.

Lebensohn and Needleman (2016) have proposed a way to enforce the micro-hard condition (eq. (12)). They ensured that no slip would develop within voxels adjacents to grain boundaries by setting a very high critical shear stress for them. This method is used in the present work to enforce the microhard, as well a the microfree interface conditions at grain boundaries. The latter is implemented by setting the material length-scale $\lambda$ to 0 on these voxels, which ensures that $\underline{\boldsymbol{m}}=0 . \Omega_{G B}$ (see fig. 5 (a) for a practical illustration) denotes the set of voxels that are in contact with a grain boundary. The two conditions are thus implemented 
as follows:

$$
\begin{aligned}
& \text { Microhard-1 : } \tau_{c}^{s}(\boldsymbol{x})=10^{5} \tau_{0}^{s} \quad \forall \boldsymbol{x} \in \Omega_{G B} \\
& \text { Microfree : } \lambda(\boldsymbol{x})=0 \quad \forall \boldsymbol{x} \in \Omega_{G B}
\end{aligned}
$$

Despite their straightforward implementation, they rely on the specification of the constitutive behavior of entire voxels, enforcing the conditions on a sub-volume rather than a surface. They are therefore likely to increase the mesh-dependence of the results, as this sub-volume size is directly proportional to the voxel size. In order to circumvent this shortcoming, we propose here two additional types of boundary conditions that do not require to modify the behavior of a material subvolume, but solely rely on the practical evaluation of the differential operator involved in the non-local equation.

Indeed, it is recalled that computing eq. (18) on a particular domain ensures that the balance equation eq. (9) is verified over this domain. It follows that, within this domain, after eq. (11), the double-traction tensor $\underline{\boldsymbol{m}}$ is well-defined and continuous. Because of the Finite-Differences-FFT based implementation, the differentiation operator given by eq. (60) must be applied uniformly over each voxel without any distinction as to whether it belongs to a particular grain. As a result, all the grain boundaries are included within the domain over which the balance equation is verified, and hence, our implementation is equivalent to enforcing a Continuity condition for $\underline{\boldsymbol{m}}$ at grain boundaries.

A slight modification of this computation can be advantageously used to enforce a micro-hard grain boundary condition. The idea relies on a partition of the polycrystalline simulation domain $\Omega$ in $M$ subsets of non connected grains $\Omega_{i}$ (see fig. 5 (b) for an illustration). A field $\underline{\boldsymbol{H}}_{i}^{p}$ is then constructed for each subset as the restriction of $\underline{\boldsymbol{H}}^{p}(\boldsymbol{x})$ to the subset and complemented by zeros, which is formally written:

$$
\begin{cases}\underline{\boldsymbol{H}}_{i}^{p}(\boldsymbol{x})=\underline{\boldsymbol{H}}^{p}(\boldsymbol{x}) & \forall \boldsymbol{x} \in \Omega_{i} \\ \underline{\boldsymbol{H}}_{i}^{p}(\boldsymbol{x})=0 & \forall \boldsymbol{x} \notin \Omega_{i}\end{cases}
$$

Instead of a uniform evaluation of eq. (60), we propose to evaluate it for each $\underline{\boldsymbol{H}}_{i}^{p}$ and sum the results in order to construct the final microstress (eq. (64)). The differentiation operator is thus evaluated identically as previously in the bulk of the grains, however, it only involves values defined within the grain and zeros to compute backstresses at grain boundaries. This process ensures that the balance equations are verified within the bulk of each grain without imposing the continuity of $\underline{\boldsymbol{m}}$ at grain boundaries. Besides, the computed back stresses will be consistent with a $\underline{\boldsymbol{H}}^{p}$ field vanishing at the boundary of each grain, mimicking the micro-hard condition. This interface condition is denoted the microhard-2 boundary condition:

$$
\text { Microhard-2 : } \underline{\boldsymbol{s}}(\boldsymbol{x})=-\lambda^{2} \pi_{0} \sum_{i=1}^{M} \mathbb{I}_{\Omega_{i}}(\boldsymbol{x}) \operatorname{curl}\left(\operatorname{curl}\left(\underline{\boldsymbol{H}}_{i}^{p}(\boldsymbol{x})\right)\right)
$$

where $\mathbb{I}_{\Omega_{i}}$ is the indicator function of the set $\Omega_{i}$. As the continuity and microhard-2 conditions do not rely on the specification of a condition over a sub-volume, they are believed to be more consistent and less voxel size dependent. Note that a similar strategy could be thought of for the micro-free condition, by prescribing $\underline{\boldsymbol{M}}=0$ outside of each partition to approximate the interface condition. Though, such strategy has not been investigated in the present work.

It must be noted though, that the microhard-2 condition is more computationally expensive, as it requires $M$ evaluations of the non local equation eq. (60). In order to mitigate this increased cost, the smallest partition of $\Omega$ in non-connected grains subsets should be used, which ultimately reduces to a graph coloring problem. For a two dimensional polycrystal, the 4 colors theorem (Appel et al., 1977) ensures that the minimal partition involves 4 sets of grains. A coloring algorithm to compute such partitions has been developed. For the sake of brevity, it will not be presented in detail. Note however that it is based on a greedy algorithm that randomly colors grains, creating new colors on the fly as needed to avoid neighbors of the same color. Colorings are produced until an optimal one is found. To accelerate the search, before 
creating a new color, the algorithm tries to invert a Kempe chain (in this context, it is a maximal connected chain of grains of two colors defining the chain ${ }^{5}$ ) starting from this grain. As illustrated in fig. 5(b), it delivers one of the minimal 4-colorings of a $2 \mathrm{D}$ polycrystals. It has also been tested on a 64 grains 3D polycrystal (obtained from a Voronoi tessellation), and provided 7 non connected partitions.

The impact on slip localization and on GND induced hardening of these various conditions will be discussed in section 6.1.

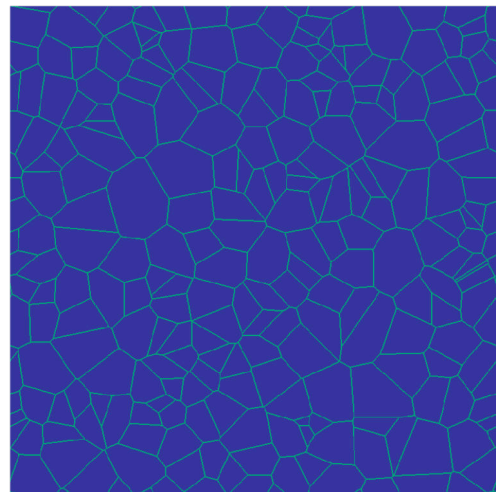

(a) $\Omega_{G B}$
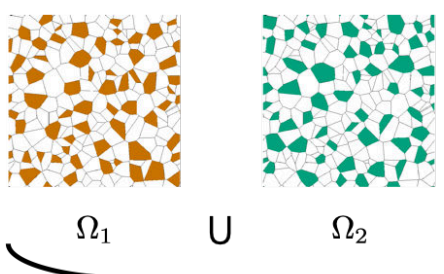

U

$\Omega_{2} \quad U$
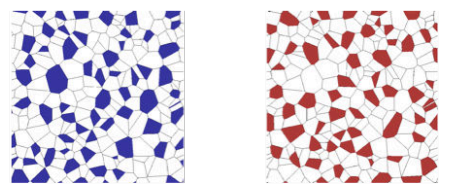

$\Omega_{3}$

U

$\Omega_{4}$

$\Omega$

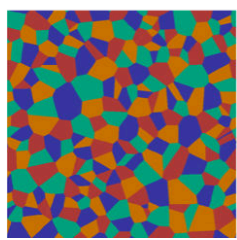

(b) Polycrystal partition

Figure 5: (a) Grain boundary voxels on which the microhard 1 and microfree boundary conditions are enforced (the set $\Omega_{G B}$ ) are represented in light blue for a 2D polycrystalline unit cell (b) Partition of a 2D polycrystalline unit cell in 4 non-connected grains subsets used to apply the microhard-2 grain boundary condition.

\subsection{Numerical validation with respect to analytical solution}

In order to validate the implementation, the case of the ideal individual kink band, modeled in section 2.2 , is simulated. With regard to the main focus of the present paper, the softening case would be the relevant case to study for numerical validation. It is deeply investigated in section 4.2. Results show that this case exhibits interesting instabilities, which however prevent a rigorous comparison with analytic results. For this reason, the stable hardening case has been chosen to demonstrate the validity of our numerical implementation.

It is recalled that the geometry is schematized in fig. 2 (b). The elastic behavior is kept linear isotropic, and the plastic behavior linearly hardening, as described by eqs. (27) and (28), and is associated to the flow rule governed by eq. (24), where the Norton exponent and coefficient values have been set in order to minimize rate-dependence. The material parameters used for this simulation are $H=1000 \mathrm{MPa}, n=100$, $K=0.5 \mathrm{MPa} \mathrm{s}^{1 / \mathrm{n}}, \tau_{0}=10 \mathrm{MPa}, \pi_{0}=H, \mu=38.6 \mathrm{GPa}$. In order to compare the purely local (CCP) to the non-local (SGP) formulation, 2 values of $\lambda$ are tested: $\lambda=0$ and $\frac{\lambda}{L}=\frac{1}{20}=0.05$.

The $x_{m}$ direction is discretized with 80 voxels, and the rigid layers are modeled by layers of 2 voxels width, purely elastic with a shear modulus $10^{5}$ higher than the one in the kink band. The unit cell is submitted to the mean shear $\bar{\gamma}^{c}=0.01$, which corresponds to the mean shear $\bar{\gamma}=0.01 \times \frac{80}{80-4} \approx 0.0105$ applied to the KB. Results are displayed in fig. 6.

\footnotetext{
${ }^{5}$ See Kempe (1879) and Appel et al. (1977) for more details on this concept central to the 4-color theorem demonstration, that are beyond the scope of this work.
} 
In the local case $(\lambda=0$, i.e. $\mathrm{CCP})$, the $\mathrm{KB}$ case is equivalent to the $\mathrm{SB}$ case, whose solution is given by a constant value $\gamma=\left(\bar{\gamma}-\frac{\tau_{0}}{\mu}\right) /\left(1+\frac{H}{\mu}\right)$. Considering the material coefficients values, the conditions $\mu \gg \tau_{0}$ and $\mu \gg H$ are verified, hence the solution should be $\gamma \approx \bar{\gamma} \approx 0.0105$, which is in agreement with the numerical solution represented by the blue curve in fig. 6 . In the non local case $(\lambda / L=0.05)$, the analytic solution is given by eq. (44), and is represented by the black dashed curve. Results show that the numerical solution, plotted in orange, agrees perfectly with this analytic solution. Hence, these results validate our numerical implementation.

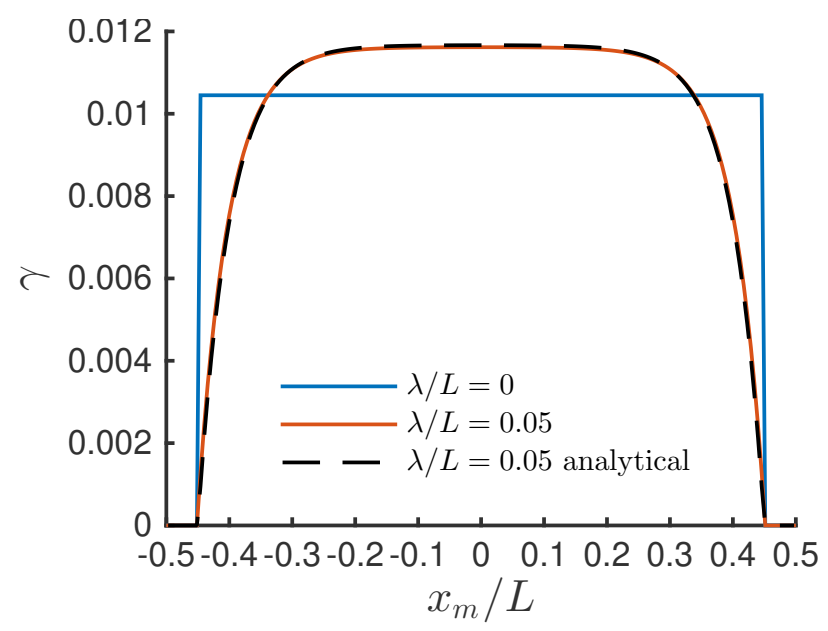

Figure 6: Ideal kink band (fig. 2(b)) simulation for $H=10 \mathrm{MPa}$. Comparison of the results for vanishing $(\lambda=0)$ vs. non-zero $\left(\frac{\lambda}{L}=\frac{1}{20}\right)$ gradient effects (grid resolution: $\left.\frac{\delta x}{L}=\frac{1}{80}\right)$.

\section{Simulations of slip and kink bands in single crystals}

In this section, the numerical implementation of the SGP model is applied to the simulation of slip localization in single crystals. These simulations will highlight the influence of the SGP framework on individual SB and KB formation. In order to avoid any confusion with the previous numerical validation case, we specify that hereafter all simulations will be based on the constitutive equations detailed in section 2.1, in particular eqs. (24) and (25) for the equations governing the evolution of softening plasticity. Additionally, note that all simulations presented in the following are carried out using strain increments of $10^{-6}$.

\subsection{Competition between slip and kink banding in an infinite single crystal}

First, a periodic homogeneous single crystal square domain with a single slip system, rotated at 45 degrees from the grid directions, is studied. The material parameters used for this simulation are $E=100 \mathrm{GPa}$ (Young's modulus), $\nu=0.3$ (Poisson's ratio), $n=20, K=1 \mathrm{MPa} \mathrm{s}^{1 / \mathrm{n}}, \tau_{0}=\pi_{0}=10 \mathrm{MPa}, \eta=0.2$, and $\gamma_{0}=0.2$. Note that this choice for $n$ and $K$ renders the material behavior almost rate-independent. The intensity of gradient effects is governed by the ratio $\frac{\lambda}{L}$.

The length of the periodic cell is denoted by $L$. It is discretized by $21 x 21$ pixels and submitted to a tensile loading in the vertical direction, up to $1 \%$ average strain, with all other components of the average stress tensor prescribed to 0 . An imperfection has been placed at the exact central voxel of the simulated unit cell, to trigger slip localization. In this voxel, the initial critical shear stress is $\tau_{0}^{\prime}=0.99 \tau_{0}$. These loading conditions and imperfection location ensure that the two potential localization bands that can form, the $\mathrm{SB}$ and the $\mathrm{KB}$, are strictly symmetric from both the point of view of the load and the structure. Hence, 
when no gradient effects are accounted for, these two bands should form simultaneously and accommodate the same amount of strain.

To begin with, a purely local simulation is carried out with $\frac{\lambda}{L}=0$. The obtained plastic slip field is shown in fig. 7 (a), and the associated lattice rotation angle field in fig. 7 (b), along with the indication of the glide direction $\boldsymbol{m}$ and normal to slip plane direction $\boldsymbol{n}$ (fig. 7 (a)). As expected, plasticity is confined into two localization bands of width 1 voxel, one being a SB, the other one being a KB. Both bands exhibit the same level of local plastic slip. A lattice rotation angle localization band is also observed in fig. 7 (b), parallel to $\boldsymbol{n}$, which is a signature of the KB. Note that the value of the rotation angle is very close to the value of the slip variable in the $\mathrm{KB}$, which validate the approximation made in section 2.2 that $\theta \approx \gamma(\sim 0.34)$ in the $\mathrm{KB}$ at incipient plasticity and under the assumptions, verified here, that all plasticity related shear stress terms in eq. (49) on the right hand-side are negligible compared to the elastic shear modulus $\mu$.

Then, non vanishing gradient terms are accounted for by setting $\frac{\lambda}{L}=0.1$. The simulated plastic slip fields are presented in fig. 7 (c-d), where resp. the curl $2^{*}$ and curlcurl ${ }^{*}$ have been used to compute the backstress on the slip system (cf. section 3.2). They reveal that accounting for gradient effects has influenced the competition between the localization modes: here, the KB does not form and all plastic slip localizes in the SB. Besides, the use of the curlcurl* operator (fig (d)) results in a strongly regularized SB, that forms with a finite width and a reduced intensity. According to the theory (section 2), this is expected only for KBs. On the other hand, the SB that forms in the simulation relying on the curl $2^{*}$ operator (fig (c)) is clearly unaffected by a gradient regularization. These results are in line with those discussed in section 3.2 and Appendix A, and confirm the choice of the curl $2^{*}$ operator to compute the backstress.

To summarize, these simulations show that accounting for a non-zero energy associated to GND formation strongly affects the competition between slip and kink banding when none of them is structurally favored. In addition, they confirm the crucial importance of using a double curl operator that preserves the physical properties of vanishing GND density and associated backstress in SBs.

\subsection{Simulation of ideal kink banding}

Next, the numerical simulation of ideal KB formation in the case of a softening behavior are presented. This case has been studied analytically in section 2.2 and is depicted in fig. 2 (b). This study aims at illustrating how the SGP model affects the numerically predicted physical characteristics of simulated KBs.

Again, a single crystal with one active slip system is modeled, as a layer of width $L$, comprised between two layers of a purely linear isotropic elastic material. In the analytic study, the strain in these elastic layers is neglected. In order to account for this assumption, the a shear modulus is set $10^{5}$ higher in the layers than in the kink band. The band is submitted to a mean shear loading $\bar{\gamma}=0.01$. The unit cell is discretized with a regular grid of $361 \times 361$ voxels, with 340 voxels in the $\mathrm{KB}$ width $^{6}$. The material coefficients are identical to those presented in section 4.1, except for $\eta$ that will be varied, and $\lambda$ set to $\frac{\lambda}{L}=0.05$.

The results are compared to the analytic solution derived in section 2.2 , although simulations rely rather than the perfectly plastic and linearly softening model of section 2.2, on eqs. (24) and (25). Indeed, Scherer et al. (2019) have shown in a similar context that, the prediction of the linear model is a good approximation for the exponential one when using in the analytic formulas the tangent modulus $H^{\prime}$ of the softening curve. After eq. (25), this modulus is given by $\left|H^{\prime}\right|=\frac{\Delta \tau}{\gamma_{0}}$. Thus, with $\pi_{0}=\tau_{0}, \Delta \tau=\eta \tau_{0}$, and using eq. (48), the kink band width can be approximated by:

$$
\omega_{b} \approx 2 \pi \lambda \sqrt{\frac{\gamma_{0}}{\eta}}
$$

Note that it depends on both the softening rate through $\gamma_{0}$, softening intensity through $\eta$, and the material length scale $\lambda$.

\footnotetext{
${ }^{6}$ Note that due to the periodic $\mathrm{BC}$, this amounts to a laminate microstructure alternating the two materials on 340 and 21 voxels layers
} 


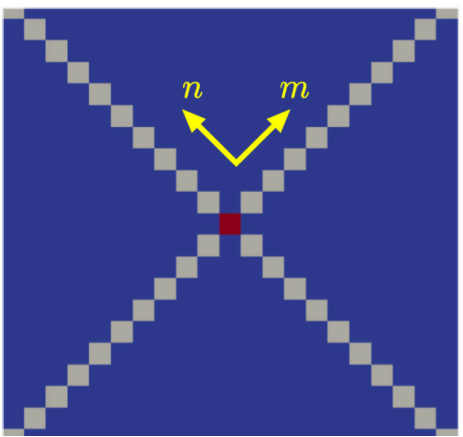

(a) $\lambda / L=0$

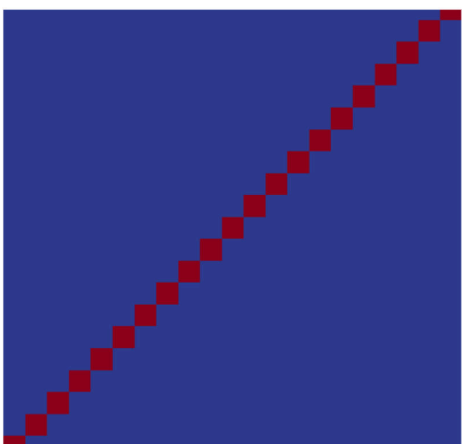

(c) $\lambda / L=0.1$
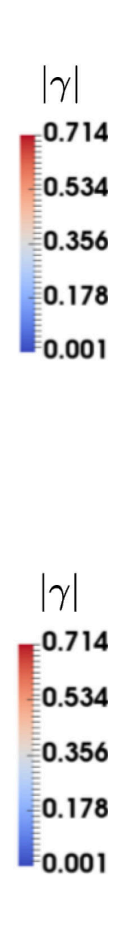

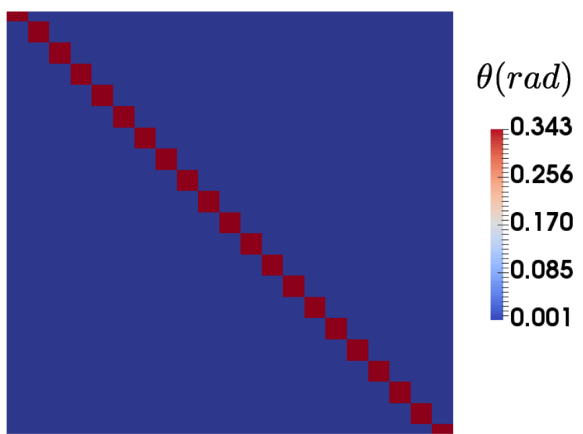

(b) $\lambda / L=0$

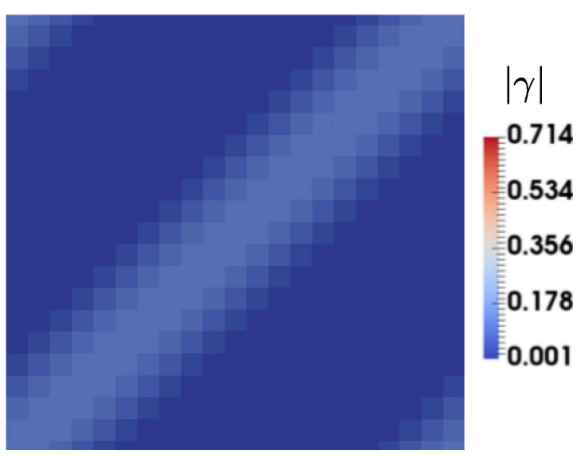

(d) $\lambda / L=0.1$

Figure 7: Plastic slip (a,c,d) and lattice rotation angle (b) fields simulated in a periodic single crystal with one active slip system under traction, without gradient effects (a-b), and with gradient effects (c-d). The double curl operator used for the simulation is curl $2^{*}$ in (c), and curlcurl* in (d). $L$ is the length of the unit cell edges (21x21 voxels).

Like in the previous case, an imperfection has been placed at the exact central voxel of the simulated unit cell, for which the initial critical shear stress is $\tau_{0}^{\prime}=0.99 \tau_{0}$. This defect helps to numerically trigger the plastic instability, and ensure that the $\mathrm{KB}$ will form at the center of the unit cell, hence enforcing the symmetry condition considered in the reference analytic problem (section 2.2).

The mechanical fields simulated for $\eta=0.05$ and $\eta=0.2$ are presented in fig. 8 . The first striking feature, at variance with the analytic modeling, is the non homogeneity along the $x_{n}$ direction observed on the field $\gamma$. For $\eta=0.05$ (a), the KB has a homogeneous distribution along most of its length, but is found to decompose partially into a few intense slip bands at its center (which coincides with the imperfection location). In the case $\eta=0.2(\mathrm{~b})$, the KB is completely decomposed into a dense distribution of intense slip bands. Additionally, the slip intensity of these slip bands forms an odd periodic pattern, that is attributed to numerical instabilities. Note that in both cases, plastic slip is confined into a band of finite width, wider in the $\eta=0.05$ than in the $\eta=0.2$ case, which is in qualitative agreement with eq. (65). According to this formula, the band width with $\eta=0.05$ should be twice the width obtained with $\eta=0.2$. Given the inhomogeneity of the field, a proper measurement cannot be achieved but the band width seem visually in agreement with this quantitative prediction.

In order to illustrate the influence of the imperfection on the results, a slip field simulated with $\eta=0.2$ and 5 imperfections randomly located on the line $x_{m}=0$ is shown on (e). In this case, the KB is also decomposed into a dense distribution of parallel SBs whose slip intensity exhibits a similar odd pattern. However, these features are less noticeable than in the single imperfection case. Most strikingly, five intense SBs stand out in the KB, at the exact locations where the imperfections where generated (circled in yellow). Within them, the intensity of plastic slip is much higher than in the rest of the KB, and they extend much 

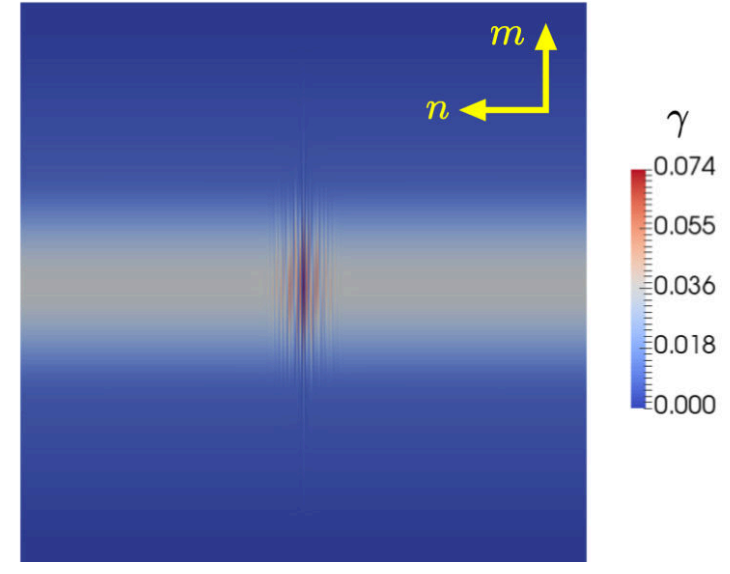

(a) $\eta=0.05$

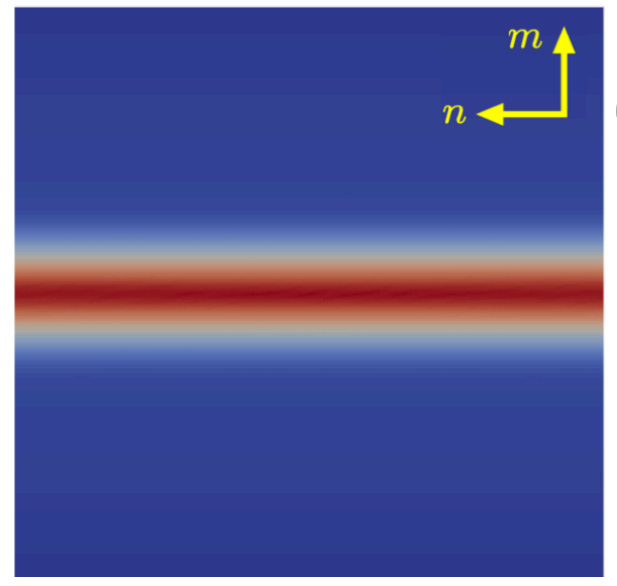

(c) $\eta=0.2$

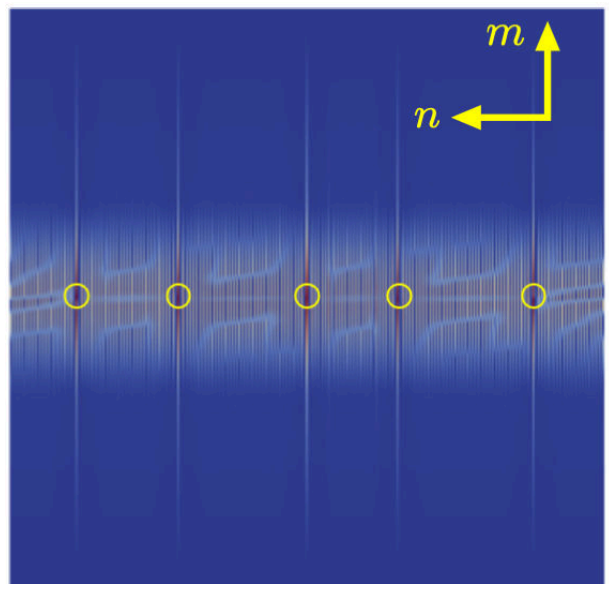

(e) $\eta=0.2 \& 5$ imperfections

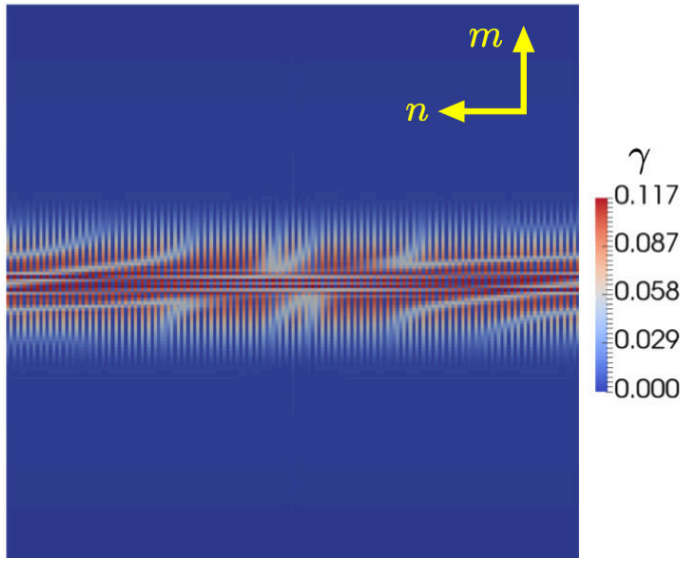

(b) $\eta=0.2$

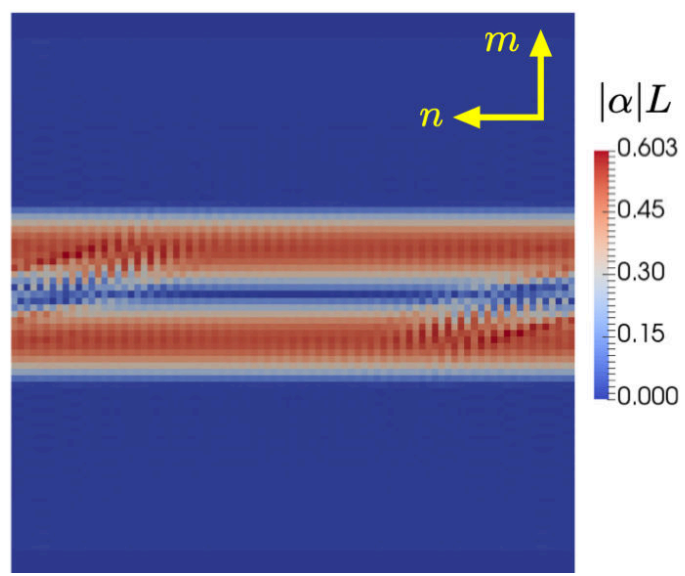

(d) $\eta=0.2$

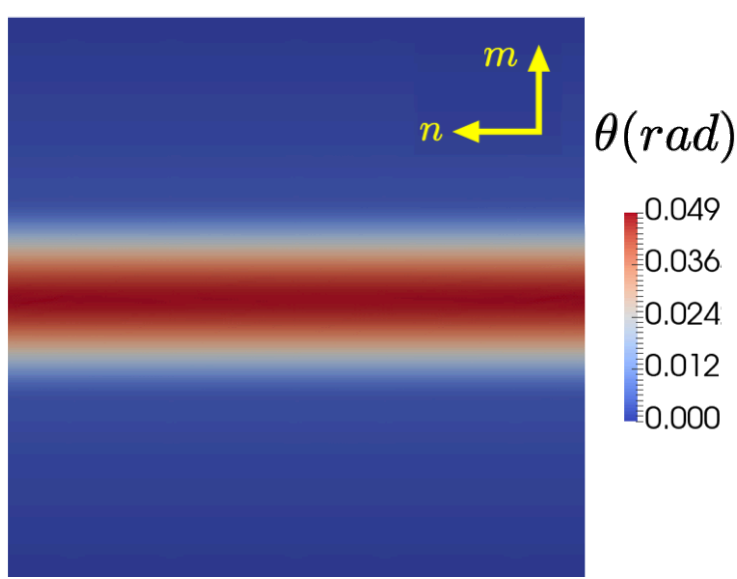

(f) $\eta=0.2 \& 5$ imperfections

Figure 8: Simulated ideal kink band (see schematization in fig. 2 (b) slip fields for two different softening intensities (a-b-e), lattice rotation angle field (c-f) and dimensionless Nye tensor's norm (d) with $\frac{\lambda}{L}=0.05(360 \times 80$ voxels in the band). (a-d): simulations feature 1 imperfection at the center of the unit cell, (e-f): the simulation feature 5 imperfections at random positions on the line $x_{m}=0$. All simulations feature $360 \times 360$ voxels 
further in the $x_{m}$ direction than the KB width.

In contrast to $\gamma$, the lattice rotation angle field $\theta$ in the case $\eta=0.2$ (c) has a perfectly homogeneous distribution along the $x_{n}$ direction. All lattice rotation is also confined into a band of finite width, consistent with the width of the slip field (b). The same homogeneous distribution is observed on the field $\theta$ computed with 5 imperfections (f), associated to the slip field (e). Compared to the field (c), obtained with a single imperfection, the rotation band appears slightly wider and slightly less intense.

The GND density field is measured here with the euclidean norm of Nye's tensor $\underline{\boldsymbol{\alpha}}$, rendered dimensionless by multiplying it by the crystal phase width $L$. It is shown in the $\eta=0.2$ and single imperfection case on figure (d). It also exhibits a homogeneous distribution along $x_{n}$, apart from a few perturbations corresponding to the similar odd fluctuations observed in the slip field (b). The figure reveals that the formation of two layers of high GND density on both sides of the KB, mimicking the GND walls associated to the formation of real KBs (fig. 1) and predicted by the theory section 2.2.

With the chosen material coefficients and for a kink band at incipient plasticity, the approximation $\theta \approx \gamma$ was proposed in section 2.2. In order to verify whether the $\theta$ field observed in the single imperfection case with $\eta=0.2$, follows the analytic prediction of eq. (47), the numerical values of $\theta$ along the $x_{m}$ direction are plotted, together with the analytic solution for $\gamma$, in Figure 9. Results show a very good agreement between these two quantities. The small discrepancy outside the band can be explained by the small elastic shear deformation occuring there in the simulation ( $\tau_{0}$ is small but not zero), whereas elastic deformations are neglected in the analytical calculation. Besides, using eq. (65) and the ratio $\frac{\lambda}{L}=0.05$, the theoretical prediction of the $\mathrm{KB}$ width can be evaluated as $\frac{\omega_{b}}{L} \approx 0.31$. Again, this numerical value is in very good agreement with the width of the rotation band observed in fig. 9 .

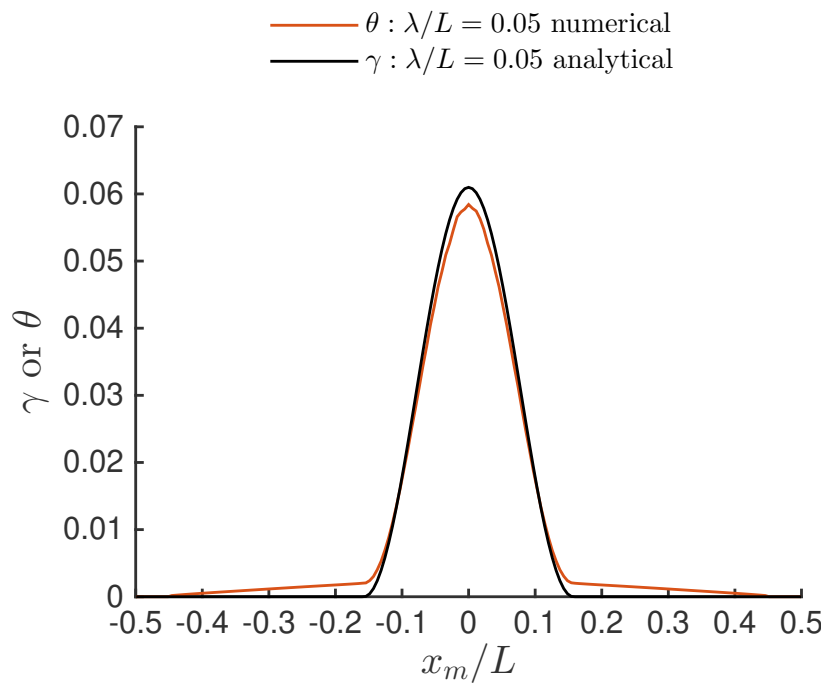

Figure 9: Analytical solution of the plastic slip profile in an ideal kink band (see section 2.2) and simulated lattice rotation angle profile associated to fig. 8 (c).

\subsection{Comment on the terminology}

Before moving to the presentation of polycrystalline simulations, some additional comments on the previous results are made regarding the designation/characterization of the localization bands observed in the simulations. According to CCP models, the two localization modes SB and KB are mutually exclusive (Marano et al., 2019): when slip localizes, locally, either a SB or a KB is formed. The results provided in this section show that this is no longer the case when using a SGP model. Indeed, they demonstrate that SBs 
can form within a KB, introducing therefore an ambiguity in the designation of the active slip localization band. In order to circumvent potential confusions, we will use the following terminology:

- Slip banding or slip localization mode: the local formation of one or several SBs (thin, parallel to active slip plane localization bands) without associated localization of lattice rotation

- Kink banding or kink localization mode: the local formation of a lattice rotation band, orthogonal to the active slip plane

According to the latter definition, kink banding may or may not be formed by a dense distribution of SBs. The presence/absence of this feature will be considered as a possible characteristic of a KB (along with width, intensity...), but will not change the name given to this localization mode (Kink banding). Finally, note that this terminology holds only in the single slip case.

\section{Simulations of slip localization in $2 \mathrm{D}$ and $3 \mathrm{D}$ polycrystals}

In this section, our massively parallel implemention of the SGP model to conduct high resolution is applied to carry out two and three-dimensional polycrystalline simulations. They are aimed at characterizing how the mechanisms of SB/KB competition and KB formation, observed in section 4, are affected by the polycrystalline microstructure. Crystal structures of increasing complexity will be considered. The results will be compared to a CCP model to evidence the influence of gradient effects.

\subsection{Simulations description}

Two periodic polycrystalline unit cells generated with a random Voronoi tesselation are considered: a 225 grains two-dimensional polycrystal, and a 64 grains three-dimensional polycrystal, voxelized with a voxel size 50 times lower than the average grain size $d_{g}$ (respectively to $750^{2}$ voxels and $200^{3}$ voxels ). A tension loading in the $\boldsymbol{e}_{3}$ direction up to $1 \%$ deformation is simulated, using increments of $10^{-6}$. Note that convergence of the stress-strain curve with respect to time/grid discretization has been ensured. Twodimensional simulations have been computed with a classical laboratory parallel computer, with 140 cores. Three-dimensional simulations where computed with 1000 cores ( $2.4 \mathrm{GHz}$ and 4.6 Go RAM per core) on the massively parallel computing facility Cobalt ${ }^{7}$. In both cases, they have been completed within less than a day.

\begin{tabular}{|c|c|c|c|c|c|c|c|c|c|c|}
\hline & $\mathrm{K}$ & $\mathrm{n}$ & $\mathrm{E}$ & $\nu$ & $\tau_{0}$ & $\Delta \tau$ & $\gamma_{0}$ & $H$ & $\pi_{0}$ & $\lambda / d_{g}$ \\
\hline $\mathrm{CCP}$ & $10 \mathrm{MPa} \mathrm{s}^{1 / \mathrm{n}}$ & 15 & $100 \mathrm{GPa}$ & 0.3 & $100 \mathrm{MPa}$ & $20 \mathrm{MPa}$ & 0.1 & $0 \mathrm{MPa}$ & $100 \mathrm{MPa}$ & 0 \\
\hline $2 \mathrm{D} \mathrm{SGP}$ & $10 \mathrm{MPa} \mathrm{s}^{1 / \mathrm{n}}$ & 15 & $100 \mathrm{GPa}$ & 0.3 & $100 \mathrm{MPa}$ & $\mathbf{5 0} \mathbf{M P a}$ & $\mathbf{0 . 0 5}$ & $0 \mathrm{MPa}$ & $100 \mathrm{MPa}$ & $\mathbf{0 . 3}$ \\
\hline $3 \mathrm{D} \mathrm{SGP}$ & $10 \mathrm{MPa} \mathrm{s}^{1 / \mathrm{n}}$ & 15 & $100 \mathrm{GPa}$ & 0.3 & $100 \mathrm{MPa}$ & $\mathbf{5 0} \mathbf{M P a}$ & 0.1 & $0 \mathrm{MPa}$ & $100 \mathrm{MPa}$ & $\mathbf{0 . 2}$ \\
\hline
\end{tabular}

Table 1: Material parameters used in the polycrystalline simulations. The coefficients varying between the CCP and SGP cases are highlighted with bold face.

Three sets of parameters are used in all simulations, and are listed in table 1. All simulations feature isotropic linear elasticity. Norton law coefficients $n$ and $K$ values are chosen in order to limit rate dependence while preserving numerical convergence. As simulated crystal systems have only one family of slip systems, superscript $s$ on flow rule and softening rule parameters is omitted in the rest of this section. The characteristic stress $\pi_{0}$ is chosen so that $\pi_{0}=\tau_{0}$. Thus the parameter controlling gradient effects is the ratio $\frac{\lambda}{d_{g}}$ (see eq. (26)). In the following, simulations with $\lambda=0$ will be referred to as CCP (classical

${ }^{7}$ http://www-ccrt.cea.fr/fr/moyen_de_calcul/index.htm 
crystal plasticity) results, and with $\lambda>0$ as SGP (strain gradient plasticity) results. In all simulations, the continuity condition for $\underline{\boldsymbol{m}}$ is prescribed at grain boundaries (cf section 3.3). The influence on the results of the other grain boundaries interface conditions (presented in section 3.3) is discussed in section 6.1.

Table 1 shows that in the SGP case, a stronger softening has been accounted for ( $\Delta \tau$ and $\gamma_{0}$ increased). This allows to compensate the hardening induced by gradient terms so that macroscopic stress-strain curves for CCP and SGP are almost superimposed (figs. 10 and 14), i.e. macroscopically equivalent CCP/SGP simulations. This allows to evaluate the physical pertinence of CCP and SGP models based solely on their local predictions.

\subsection{Single slip localization in polycrystals with a single slip plane}

First, two simple model microstructures with a single slip plane per grain are investigated: on the one hand, the two-dimensional unit-cell with a single slip plane per grain with "in-plane" crystal orientations (the slip system directions $\boldsymbol{m}$ and $\boldsymbol{n}$ are both parallel to the unit cell plane); and on the other hand, the three-dimensional unit cell with a HCP structure where only the 3 basal slip systems are taken into account. For both, crystal orientations are randomly generated. Figure 10 displays the macroscopic stress-strain curves associated to the CCP (blue curve) and SGP (dashed black curve) simulations in both cases. The orange curves show the macroscopic behavior obtained when accounting for gradient effects but without increased softening. They are plotted to highlight the macroscopic GND induced hardening that we chose to compensate by increasing the softening intensity. The good agreement of the black and blue curves is the result of the choice of coefficients presented in table 1, and demonstrate that CCP and SGP simulations are macroscopically almost equivalent in both cases.

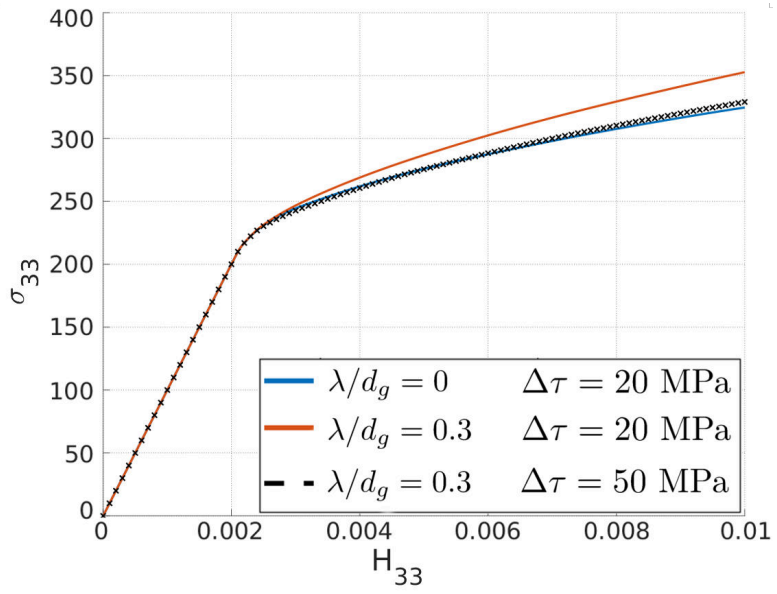

(a)

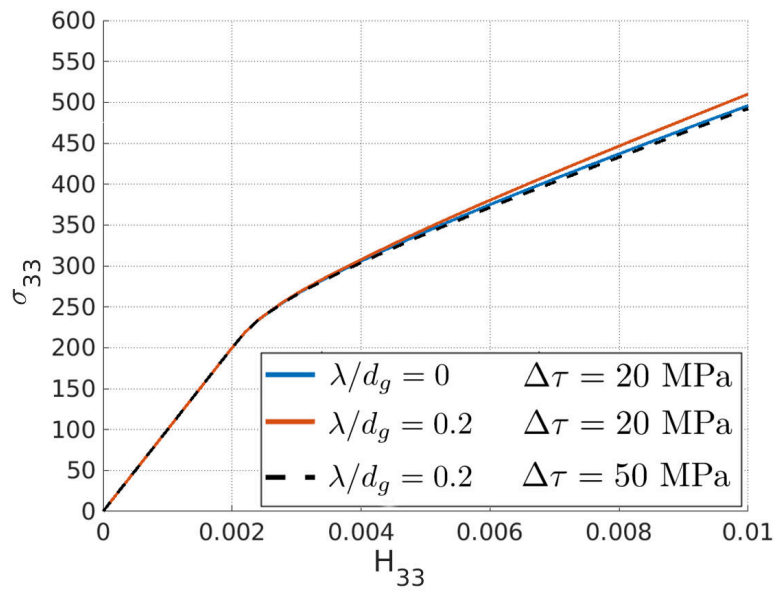

(b)

Figure 10: Stress-strain curves obtained for the 225 grains two-dimensional polycrystal with one slip system per grain (a) and the 64 grains 3D polycrystal with the $3 \mathrm{HCP}$ basal slip systems (b). CCP simulation: blue curve. SGP simulation without increased softening: orange curve. SGP simulation: dashed black curve (see table 1 first and second raws).

Simulated equivalent plastic strain fields are shown in fig. 11 (a-b), respectively fig. 12 (a-b), and lattice rotation fields in fig. 11 (c-d), fig. 12 (c-d) for the 2D, respectively 3D polycrystals. It is clear on these fields that the distribution of plastic strain at the intergranular scale is similar in both CCP and SGP cases. In general, plastic slip is intense in the same grains, and the intergranular localization networks are similar. The lattice rotation angle fields reveal localization bands for crystal rotation, indicating the grains exhibiting kink banding. Similarly, they show that rotation bands are found in the same grains in both CCP and SGP cases.

However, large differences between CCP and SGP are observed at the the intragranular scale. In the 

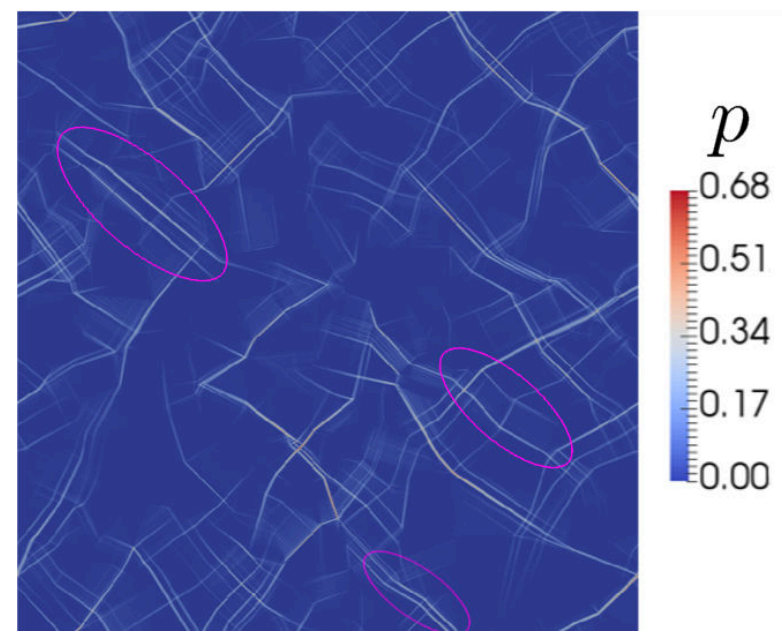

(a) $\mathrm{CCP}$

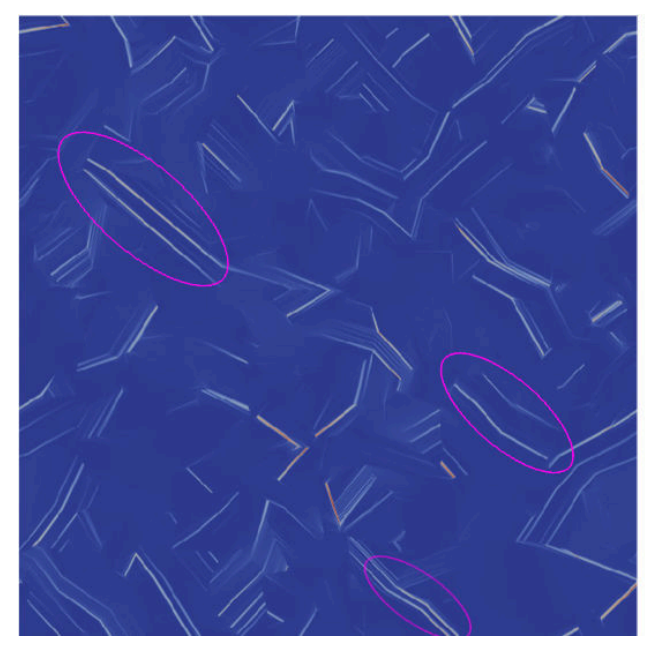

(c) $\mathrm{CCP}$

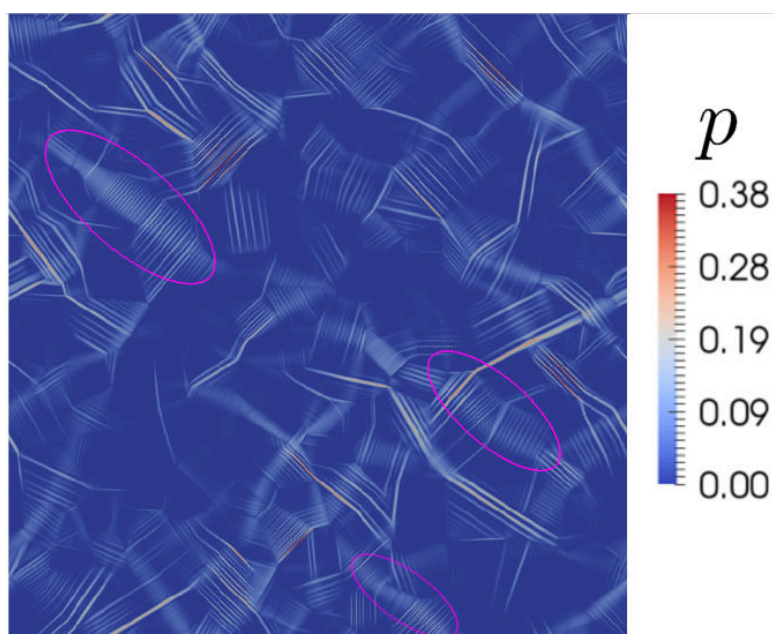

(b) SGP

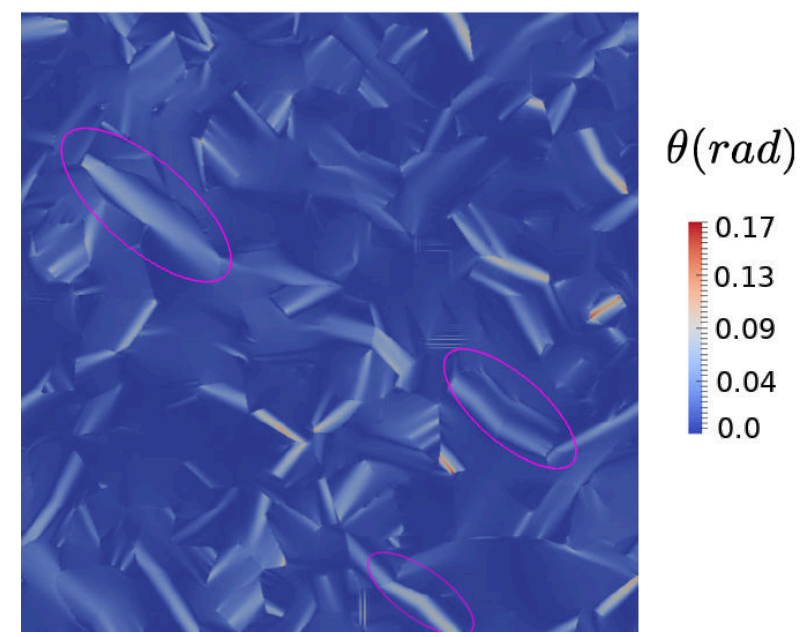

(d) SGP

Figure 11: Equivalent plastic strain (a-b) and lattice rotation angle (c-d) fields, simulated with: a(a-c) CCP model $\left(\frac{\lambda}{d_{g}}=0\right.$, $\gamma_{0}=0.1$ and $\left.\Delta \tau=20 \mathrm{MPa}\right),(\mathrm{b}-\mathrm{d})$ a SGP model $\left(\frac{\lambda}{d_{g}}=0.2, \gamma_{0}=0.05\right.$ and $\left.\Delta \tau=50 \mathrm{MPa}\right) .225$ grains $2 \mathrm{D}$ polycrystal $(562500$ voxels) with one in-plane slip system and an isotropic texture.

SGP case, the plastic slip localization bands are much more numerous and intense. In some grains, they are found to be perpendicular to the bands that formed in the same grain in the CCP case. The concerned grains all exhibit lattice rotation bands (hence KBs) in both cases. The lattice rotation bands predicted in the SGP case, display a finite width and seem to be regularized: they are wider and less intense than in the CCP case. They also appear to be formed by a dense distribution of SBs. Three regions illustrating this situation, clearly visible in the 2D case, have been circled in purple in fig. 11 . These features strongly resemble those of the individual KB simulated with SGP in section 4.2.

In order to confirm geometrically the nature of the observed slip localization bands, a relative threshold has been applied to the equivalent plastic strain field of the $2 \mathrm{D}$ case, to construct a localization map showing the areas verifying $p>3 \bar{p}$, where $\bar{p}$ is the mean of the equivalent plastic strain over the unit cell. This map is plotted in red in fig. 13, along with grain boundaries and the orientation of the slip plane in each grain (yellow lines). A careful observation reveals that almost all the bands composing the localization network 


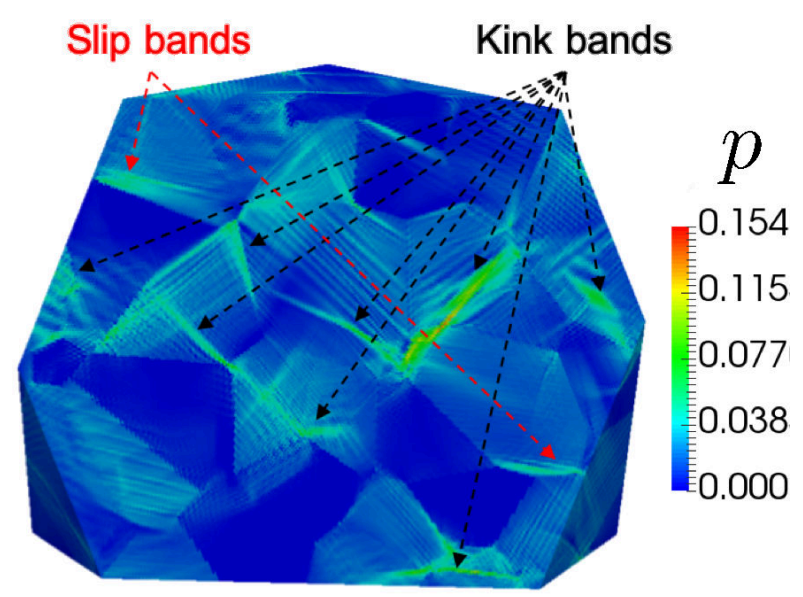

(a) $\mathrm{CCP}$

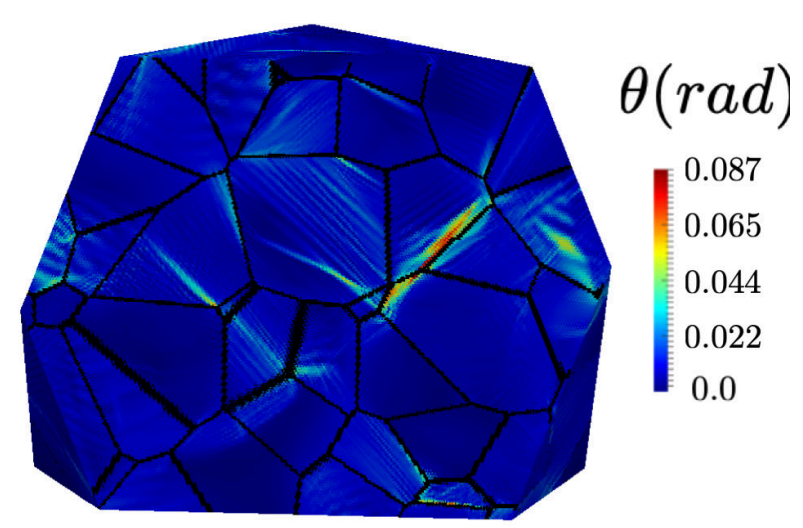

(c) $\mathrm{CCP}$

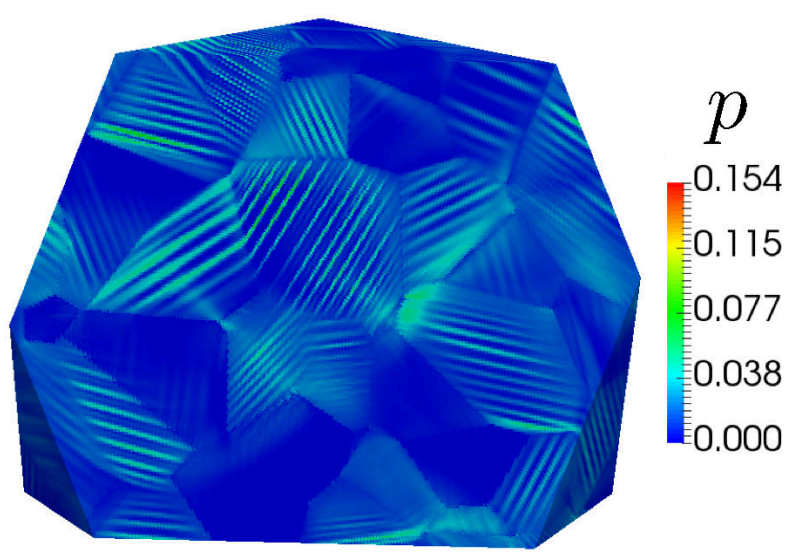

(b) SGP

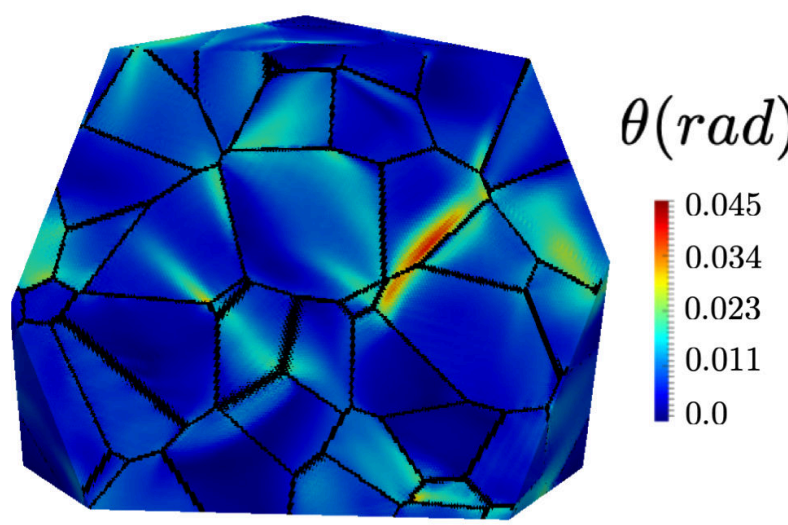

(d) SGP

Figure 12: Internal slices for equivalent plastic strain simulated with (a): a CCP model $\left(\frac{\lambda}{d_{g}}=0\right.$ and $\left.\Delta \tau=20 \mathrm{MPa}\right)$, (b) a SGP model $\left(\frac{\lambda}{d_{g}}=0.2\right.$ and $\left.\Delta \tau=50 \mathrm{MPa}\right) .64$ grains $3 \mathrm{D}$ polycrystal ( 8 million voxels) with the 3 basal slip systems of hexagonal crystal structures and an isotropic texture.

are parallel to a slip plane, and are therefore identified as SBs. In the grains exhibiting kink banding, a dense succession of SBs is observed, confirming that kink banding occurs similarly in the grains as in the case of the individual kink band (fig. 8 (b)). Yet, note that the instability leading to the decomposition in SBs is more or less pronounced, depending on the considered KB. Indeed, a few large localization bands perpendicular to a SB can be observed in fig. 13, as well as in fig. 8 (a).

To sum up, in these simulations, accounting for gradient effects does not significantly modify the plastic slip localization modes observed in the grains (slip or kink banding, in the sense defined in section 4.3). However, it strongly affects the characteristics of the formed KBs, in accordance with the results obtained for single crystals in section 4.2. This influence of the SGP model on KB characteristics, and the stronger softening accounted for in the SGP case both promote the formation of numerous, intense and parallel intragranular SBs networks. 


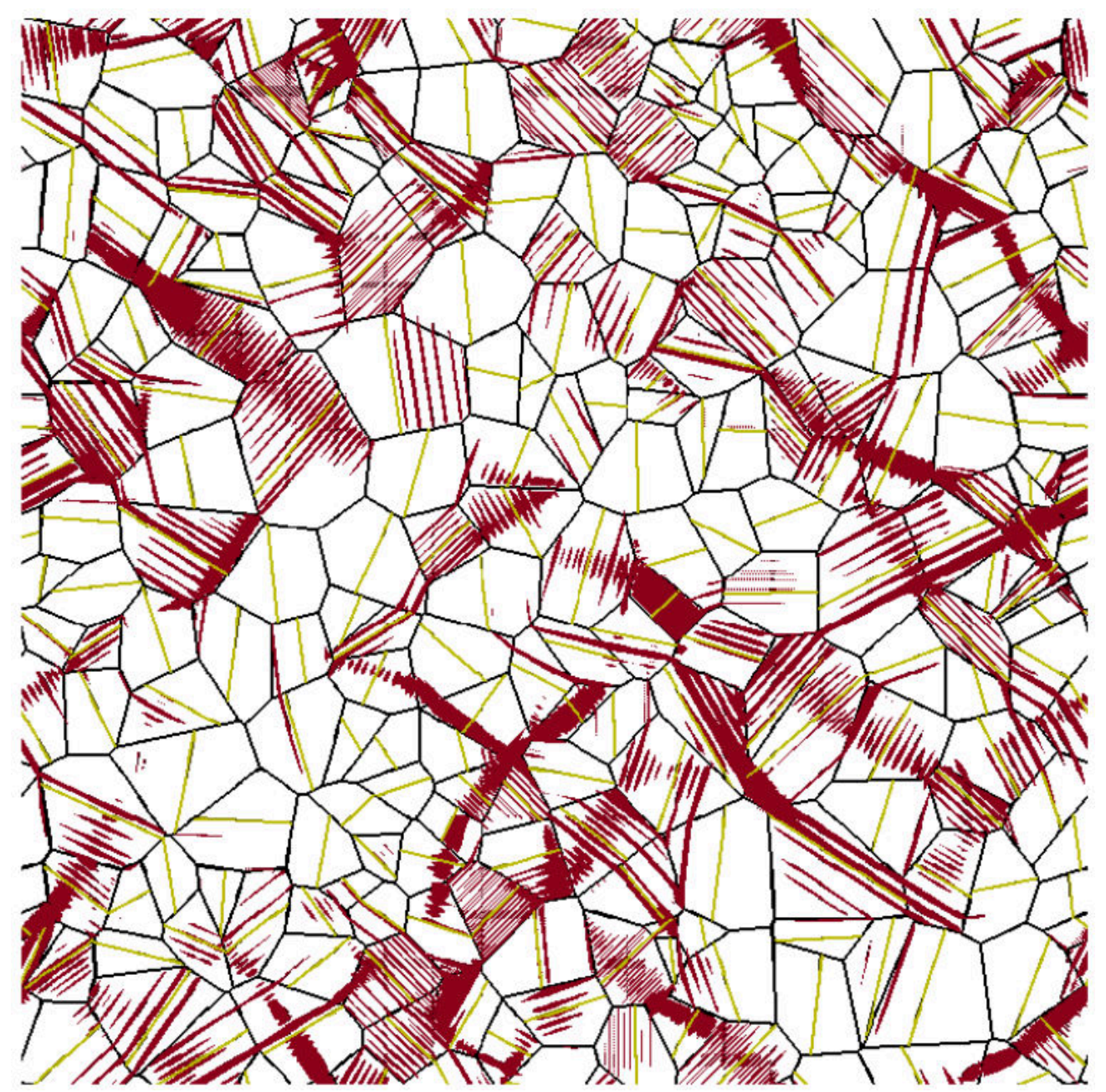

Figure 13: Localization map for the SGP simulation of the 225 grains 2D polycrystal (562 500 voxels) with one in-plane slip system and an isotropic texture. The voxels in fig. 11 (b) that have an equivalent plastic strain level at least three times above the average value are plotted here in red. The orientation of the slip system in each grain is indicated by a yellow line and the grain boundaries are represented in black.

\subsection{Simulation of slip localization in a $3 D$ FCC polycrystal}

Then, three-dimensional simulations carried out with a more complex crystal structure are presented: the $12\{111\}<110>$ FCC slip systems are accounted for. Grain orientations are again randomly generated. Figure 14 displays the macroscopic stress-strain curves, similarly to fig. 10.

The simulated equivalent plastic strain maps for the CCP and SGP are displayed in fig. 15 (a-b). Contrary to the two previous microstructures studied, for which the differences between CCP and SGP simulations were observed mainly at the intragranular scale, in the present case significant differences can be observed at the intergranular scale between both localization band networks. To investigate further the underlying mechanisms, a slice of the equivalent plastic strain, lattice rotation angle and a dimensionless Nye's tensor norm $(|\underline{\boldsymbol{\alpha}}| L$, where $L$ is the unit cell size) fields obtained in both cases are presented in fig. 16 for an in-depth analysis.

In the CCP case, three main areas constituting the localization band network at the intergranular scale can be identified. They have been circled in red on the plastic strain field of fig. 16 (a). Almost all grains 


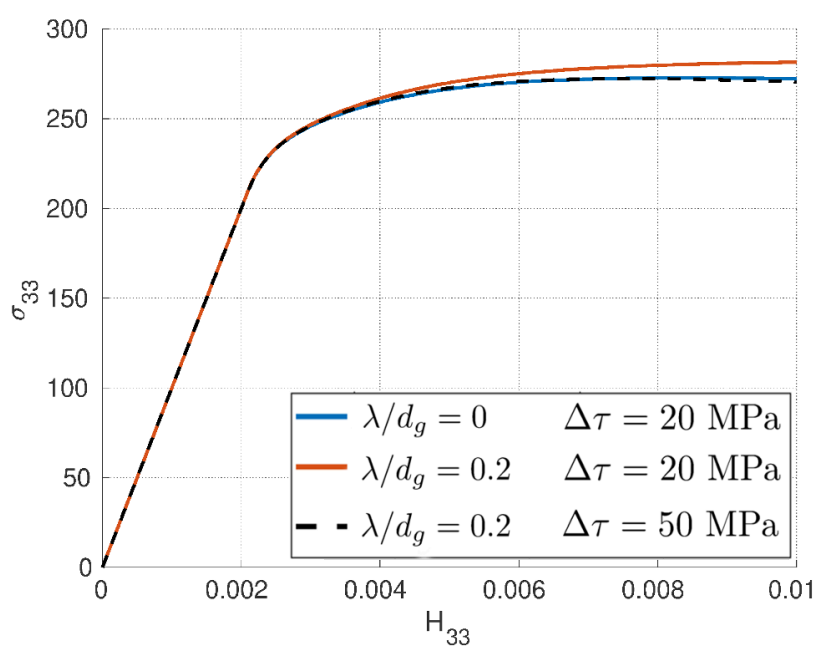

Figure 14: Stress-strain curves simulated with various values of the softening parameter and material length scale, for a 64 grains 3D polycrystal with 12 FCC slip systems and an isotropic texture. CCP simulation: blue curve. SGP simulation without increased softening: orange curve. SGP simulation: dashed black curve (see table 1).

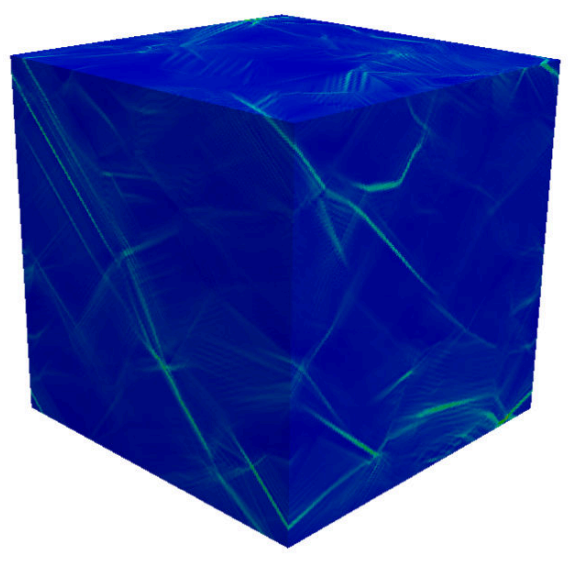

(a) $\mathrm{CCP}$

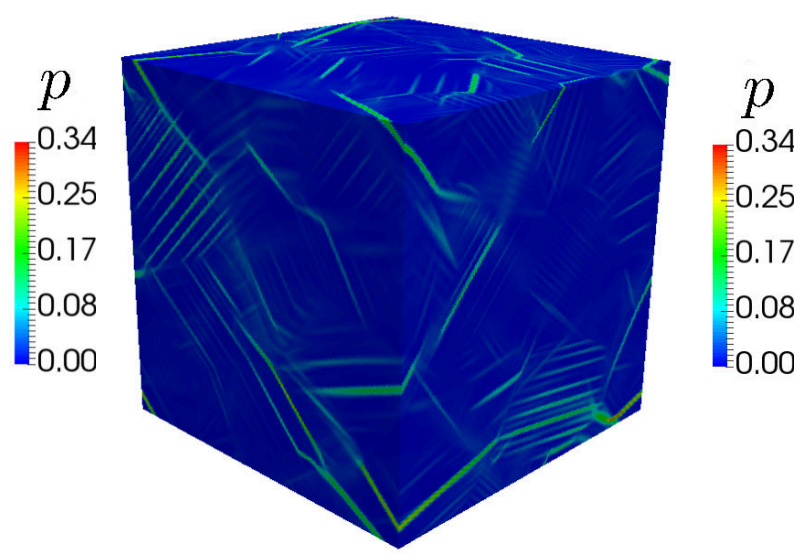

(b) SGP

Figure 15: Equivalent plastic strain fields simulated with: a CCP model $\left(\frac{\lambda}{d_{g}}=0\right.$ and $\left.\Delta \tau=20 \mathrm{MPa}\right)$, a SGP model $\left(\frac{\lambda}{d_{g}}=0.2\right.$ and $\Delta \tau=50 \mathrm{MPa}) .64$ grains 3D polycrystal (8 million voxels) with the 12 FCC slip systems and an isotropic texture.

in these areas contain one intense localization band that is connected, at both ends, to a band in another grain, across a grain boundary or a triple junction. As they involve the cooperative deformation of several grains, these areas play a central role in the deformation of the polycrystal from the structural point of view. The observation of the lattice rotation field in fig. 16 (c) in these areas (also circled in red) reveals that the majority of these bands are also lattice rotation bands, hence KBs. It can be noted that this specific polycrystalline microstructure under this loading promotes kink banding as the main intragranular deformation mode.

In the SGP case, the localization band network is strongly modified, at both intragranular and intergranular scales, as illustrated by fig. 16 (b). At the intragranular scale, the SBs of the CCP network are still present in the SGP simulation, and are found to be more intense. Besides, most intense KBs in the circled areas have been decomposed into a dense distribution of intense SBs, associated to finite width rotation bands visible in the lattice rotation angle field (d). They are found at the same locations as the rotation 


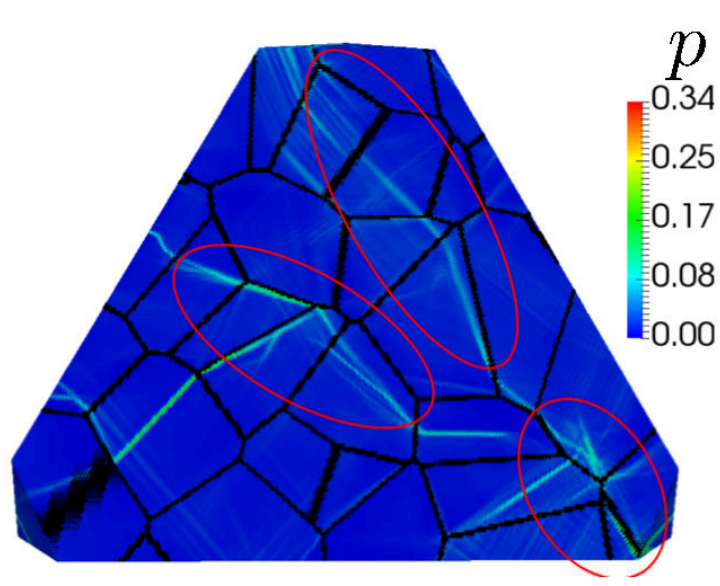

(a) $\mathrm{CCP}$

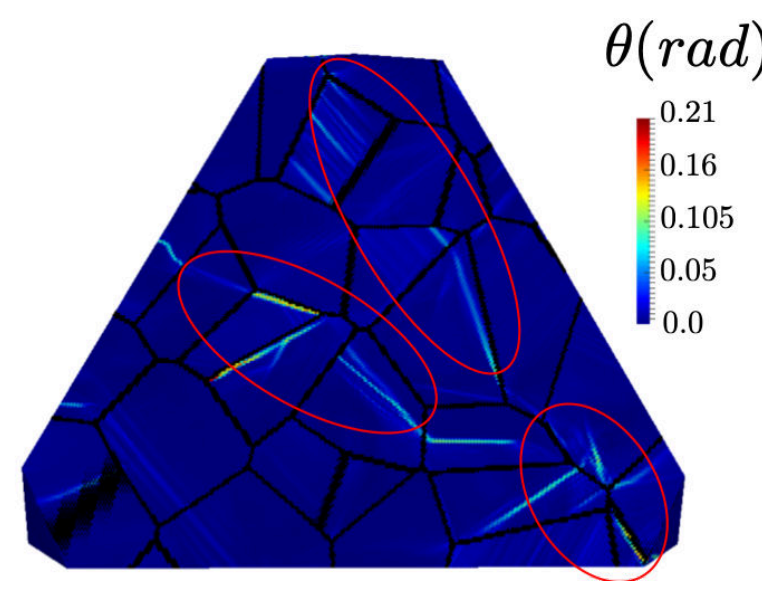

(c) $\mathrm{CCP}$

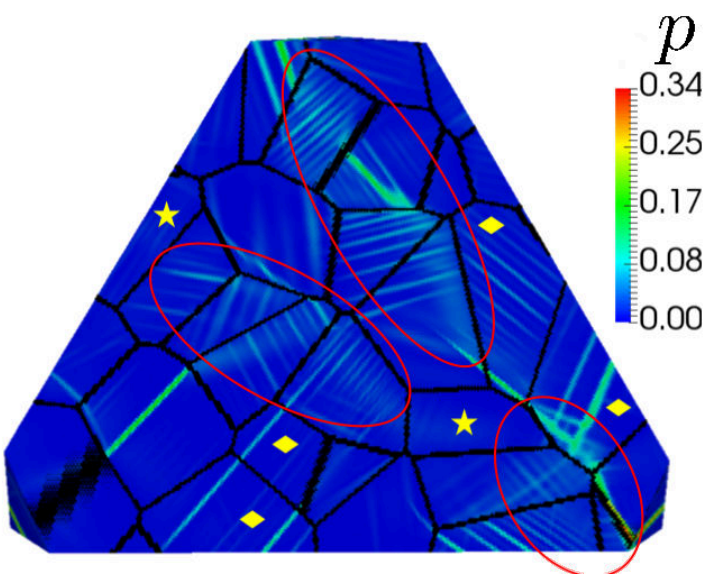

(b) SGP

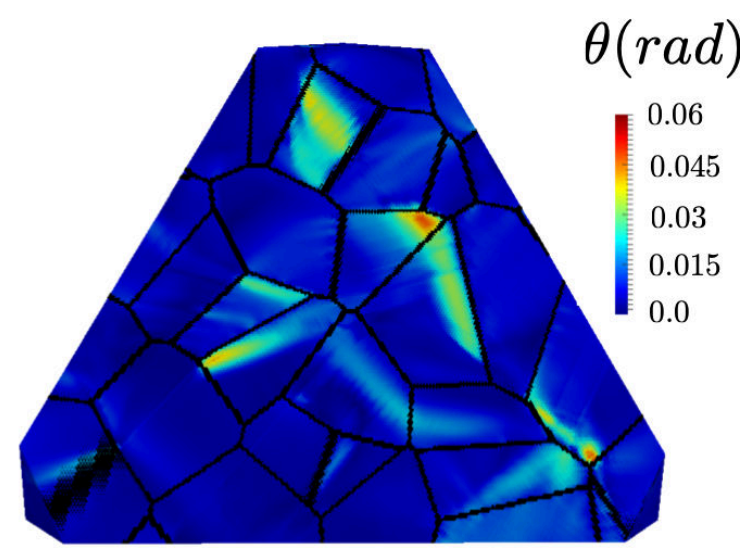

(d) SGP

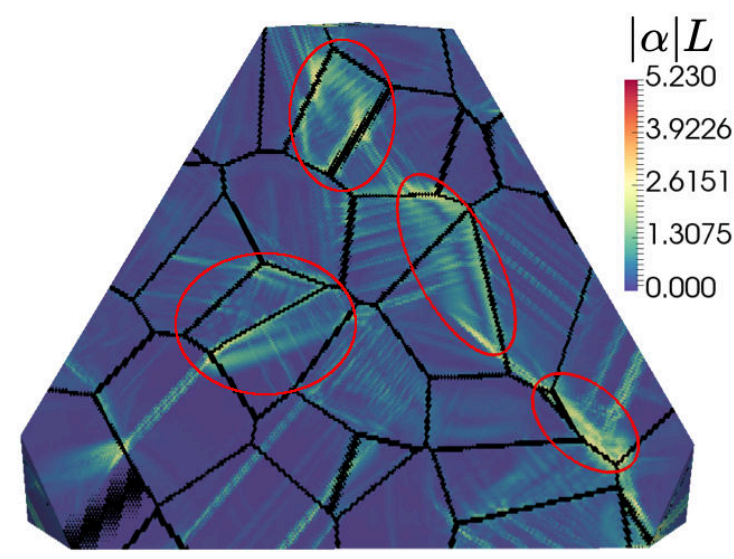

(e) SGP

Figure 16: Internal slices of the equivalent plastic strain (a-b), lattice rotation angle (c-d), and the dimensionless Nye's tensor norm (e) fields simulated with (a-c): a CCP model $\left(\frac{\lambda}{d_{g}}=0\right.$ and $\left.\Delta \tau=20 \mathrm{MPa}\right)$, (b-d-e) a SGP model $\left(\frac{\lambda}{d_{g}}=0.2\right.$ and $\Delta \tau=50 \mathrm{MPa}$ ). 64 grains 3D polycrystal (8 million voxels) with the $12 \mathrm{FCC}$ slip systems and an isotropic texture, $L$ is the unit cell size. 
bands of the CCP simulation. Clear GND walls formation on the boundaries of these rotation bands can be observed in the dimensionless Nye's tensor norm field of fig. 16 (e), circled in red. They are similar to those observed in fig. 8(d) for the individual KB simulation.

On the other hand, at the intergranular scale, it can be observed that inactive grains in the CCP case, indicated in fig. 16 (b) by a yellow diamond, are plastically activated and undergo slip banding in the SGP case. Strikingly, all of these grains contain localization bands that are connected to the localization network in the circled areas, through SBs intersection at grain boundaries. Finally, note that a few grains undergoing kink banding in the CCP case do not show signs of plastic activity in the SGP case. They are marked with a yellow star and are located outside of the circled areas. The observation of the lattice rotation field in the SGP case (d) also reveals that in these grains, lattice rotation is very moderate or vanishing, confirming that kink banding has been hindered in the SGP case.

To conclude, these results reveal a more complex picture of the influence of SGP on plastic slip localization networks than in the previous sections. Contrary to the two previous cases, here, to some extent, accounting for gradient effects hinders kink banding and promotes slip banding when compared to the CCP simulation. This phenomenon is analogous to the results of the single crystal simulations presented in section 4.1 , for which gradient effects within the single crystal hindered the KB formation to the profit of the SB. However, the competing localization modes are located here in distinct grains.

Finally, it is worth noting two observations regarding the dimensionless Nye tensor norm field in fig. 15 (e). First, areas exhibiting the highest GND density are found at the intersection between localization bands and grain boundaries, and not on KBs boundaries. Second, SBs are also visible on this field, although they should exhibit a zero GND density according to the theory. This field has been computed by applying the curl ${ }^{*}$ operator on $\underline{\boldsymbol{H}}^{p}$ in a post-processing step. As discussed in section 3.2 and Appendix A, this operator computes a non zero $\operatorname{curl}\left(\underline{\boldsymbol{H}}^{p}\right)$ for SBs not aligned with the discretization grid directions. The observation of SBs on fig. 15 (e) is thus a post-processing artifact.

\section{Discussion}

\subsection{Grain Boundary higher order interface conditions}

Here we discuss the ability of our numerical implementation to account for various boundary conditions and their influence on GB induced hardening, as well as their influence on the simulated slip localization band networks. All the results presented and discussed here are obtained with a 64 grains two-dimensional polycrystalline microstructure similar to the one presented in section 5.2 (1 in-plane slip system per grain), and with the materials coefficients listed in table 2 .

\begin{tabular}{|c|c|c|c|c|c|c|c|c|c|}
\hline $\mathrm{K}$ & $\mathrm{n}$ & $\mathrm{E}$ & $\nu$ & $\tau_{0}$ & $\Delta \tau$ & $\gamma_{0}$ & $H$ & $\pi_{0}$ & $\lambda / d_{g}$ \\
\hline $10 \mathrm{MPa} \mathrm{s}^{-\mathrm{n}}$ & 15 & $100 \mathrm{GPa}$ & 0.3 & $100 \mathrm{MPa}$ & $20 \mathrm{MPa}$ & 0.1 & $0 \mathrm{MPa}$ & $100 \mathrm{MPa}$ & 0.2 \\
\hline
\end{tabular}

Table 2: Material parameters used for the polycrystalline CCP simulations

To begin with, the convergence w.r.t. the number of voxels is studied. Indeed, the different conditions described in section 3.3, involve for some of them the modification of the mechanical behavior of grain boundary voxels, and are thus expected to exhibit a stronger grid dependence. Figure 17 presents the stress-strain curves obtained with these conditions for various values of the ratio between the grain size $d_{g}$ and the voxel size $\delta_{x}$.

fig. 18 (a) and (b) reveal a very small grid dependence, with converged results for $d_{g} / \delta_{x}=50$, for both continuity $\underline{\boldsymbol{m}}$ (see section 3.3) and microfree (eq. (62)) boundary conditions. In both cases, increasing the grid resolution tends to raise stress values. Microhard condition results obtained with both proposed implementations, microhard-1 (eq. (61)) and microhard-2 (eq. (2)), are plotted in fig. 18 (c). They clearly show that the microhard-2 grid dependence is similar to those of the continuity and microfree conditions. 


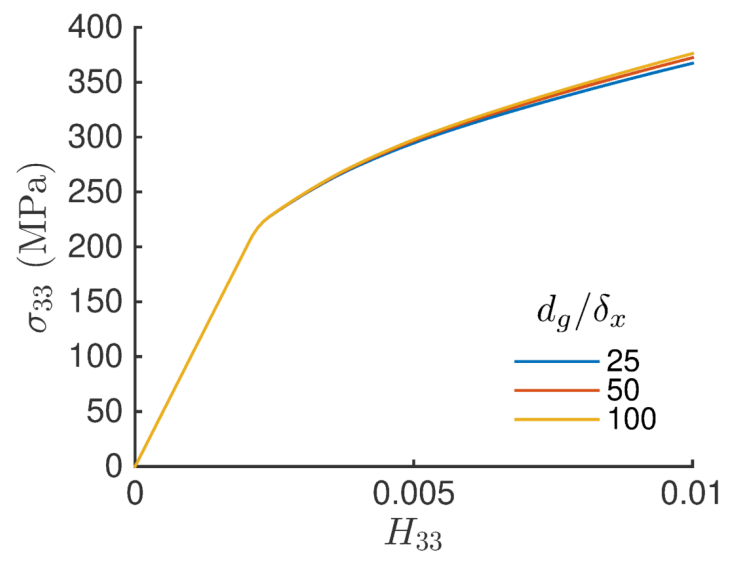

(a) continuity

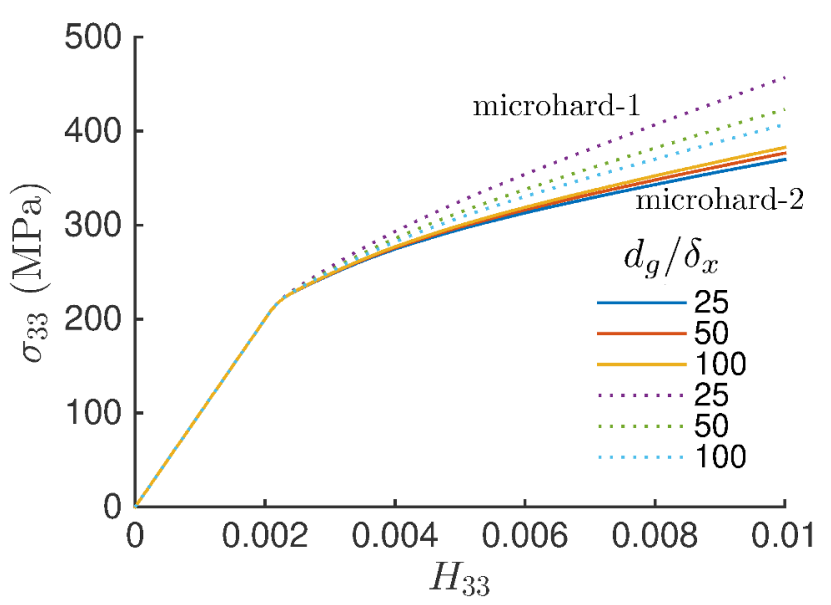

(c) microhard-1/2

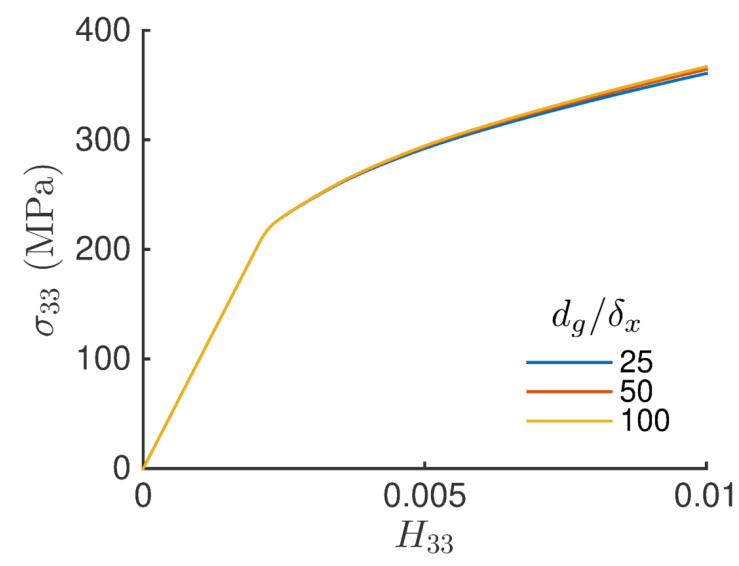

(b) microfree

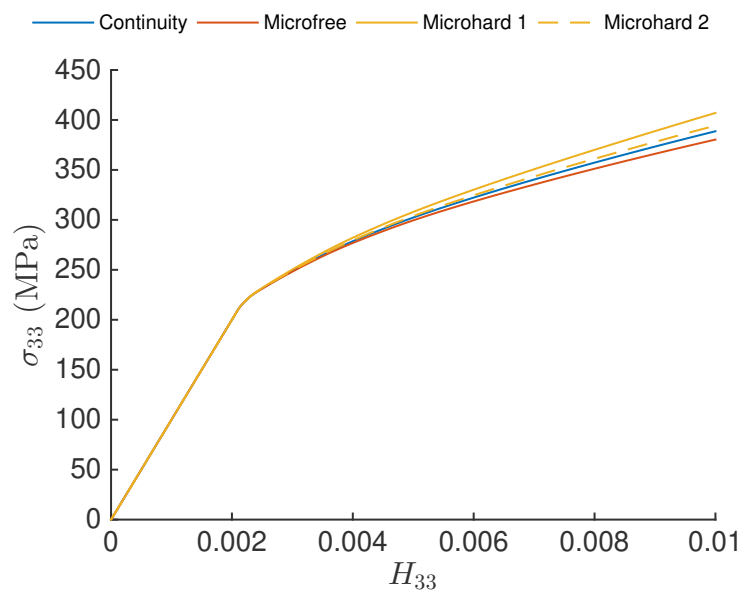

(d) all $\mathrm{BC}, d_{g} / \delta x=50$

Figure 17: Study of convergence w.r.t. the number of voxels of the macroscopic stress-strain curves obtained for: (a) for the continuity of $\boldsymbol{m}$ condition (b) for the microfree condition and (c) the two microhard BC (dotted lines correspond to the microhard-1 condition, the full lines to the microhard-2 condition). (d) Stress-strain curves for all grain BC with $\frac{d g}{\delta_{x}}=50$. All cases feature $\frac{\lambda}{d_{g}}=0.2$, and a 64 grain $2 \mathrm{D}$ polycrystal $\left(200^{2}, 400^{2}, 800^{2}\right.$ voxels $)$.

On the contrary, microhard-1 condition exhibit a pronounced and inverse dependence: a finer resolution decreases stress values with much stronger grid dependence (clearly not converged even for $d_{g} / \delta_{x}=100$ ). Note however, that both series of curves seem to converge towards the same solution, which indicates that both implementations of the microhard condition tend to predict the same amount of GB induced hardening.

In view of these results, the microhard-2 implementation is found to be more consistent numerically to prescribe the microhard condition than the microhard-1 formulation that has been proposed by Lebensohn and Needleman (2016). Moreover, it appears physically more appropriate as it avoids to prescribe a purely elastic behavior to some voxels, which adds an artificial hardening source associated to the elastic stresses developed in these voxels. A similar strong grid dependence could be expected as well for the microfree condition, as it relies on a condition prescribed on the mechanical behavior of a set of voxels. However, as this condition induces a very low GND induced hardening, the effect on the stress-strain curve is limited and, therefore, the grid dependence as well. Hence, this implementation of the microfree condition is less sensitive than the microhard-1 for this SGP theory. 
We now investigate the physically consistency of the GND induced hardening. Figure 17 (d) shows a comparison of all conditions for the same resolution $\left(d_{g} / \delta_{x}=50\right)$. Classical results are reproduced: as expected, the microfree condition is the softest one, both microhard conditions are the hardest. The continuity condition yields an intermediate hardening level. Then, we consider the quantity $\Delta \sigma_{1 \%}\left(\lambda / d_{g}\right)=$ $\sigma\left(1 \%, \lambda S / d_{g}=0\right)-\sigma\left(1 \%, \lambda / d_{g}=0\right)$ as a measure of the GND induced hardening. For a given value of $\lambda$, it compares the final macroscopic stresses obtained after $1 \%$ strain in the CCP and the SGP case associated to $\lambda$. It is plotted in fig. 18 as a function of $\lambda$ for the various boundary conditions. A linear dependence of GND induced hardening with the material length scale is observed here for all conditions. This is a classical result for a model based on a quadratic potential and Gurtin's SGP theory (Cordero et al., 2010).

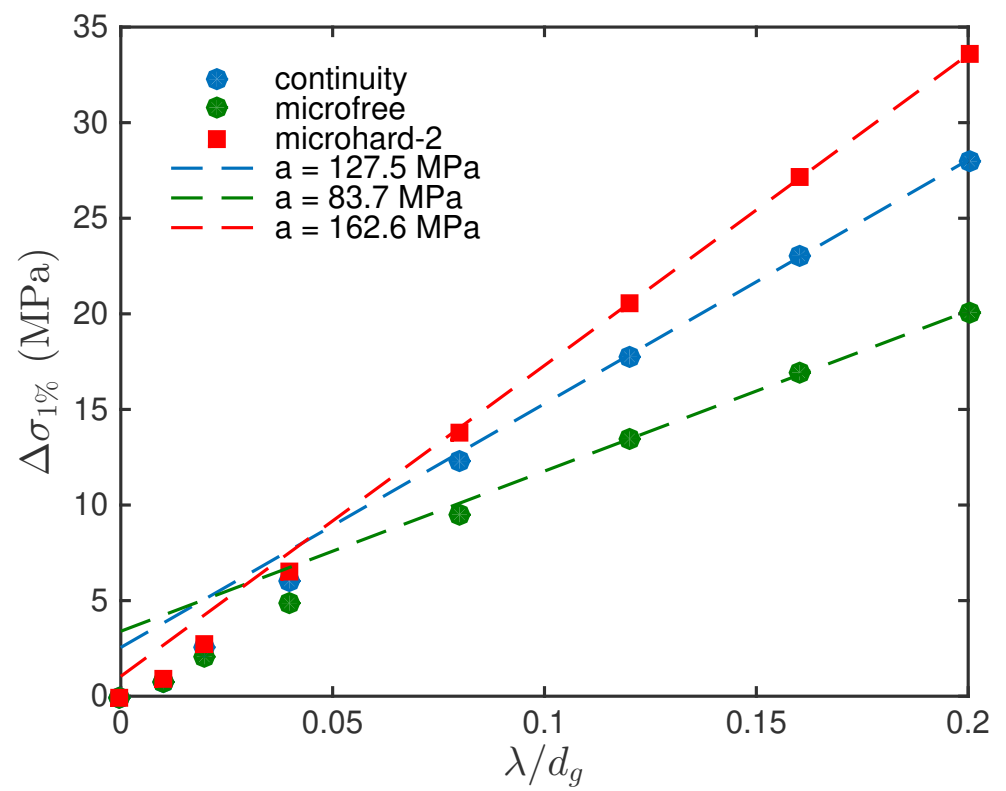

Figure 18: Comparison of the overall stress increase at $1 \%$ strain $\Delta \underline{\boldsymbol{\sigma}}_{f}=\underline{\boldsymbol{\sigma}}\left(\frac{\lambda}{d_{g}}\right)-\underline{\boldsymbol{\sigma}}(\lambda=0, H=0.01)$, in function of $\frac{\lambda}{d_{g}}$, depending on the BC enforced at grain boundaries and for $\frac{d_{g}}{\delta_{x}}=50$ (225 grain $2 \mathrm{D}$ polycrystal, $750^{2}$ voxels). The points are the values computed from numerical simulations. $a$ indicates the slope of the dashed straight lines that approximate the linear evolution of the increase in stress with the material length scale.

Finally, the influence on the localization bands patterns is discussed. Four high resolution simulations $\left(d_{g} / \delta_{x}=100\right)$ with each one of the three grain boundary conditions have been performed. The associated equivalent plastic strain fields are shown in fig. 19 (a-c). They clearly show that the interface conditions enforced at grain boundaries do not influence the localization network: the same grains are plastically active and exhibit very similar slip localization patterns. Note yet that they affect the intensity of slip localization: the plastic strain level in the bands is lower for the microhard condition than for the two others. Once again, the continuity condition yields an intermediate level of plastic strain within the bands. To provide a quantitative illustration, the distributions of high equivalent plastic strain values (where $p>0.05$ ) in these three fields have been plotted on fig. 19 (d). The conservation of the localization network for all three conditions implies that all the observations and conclusions drawn from the results presented in section 5 do not depend on the conditions enforced at grains boundaries. This holds at least for the implementation proposed in the present work.

\subsection{Physical relevance of simulated kink bands}

From a physical point of vue, KBs are single slip localization modes that locally accommodate shear strain through the local bending of crystal lattice planes, forming a lattice rotation band. Their formation involves 


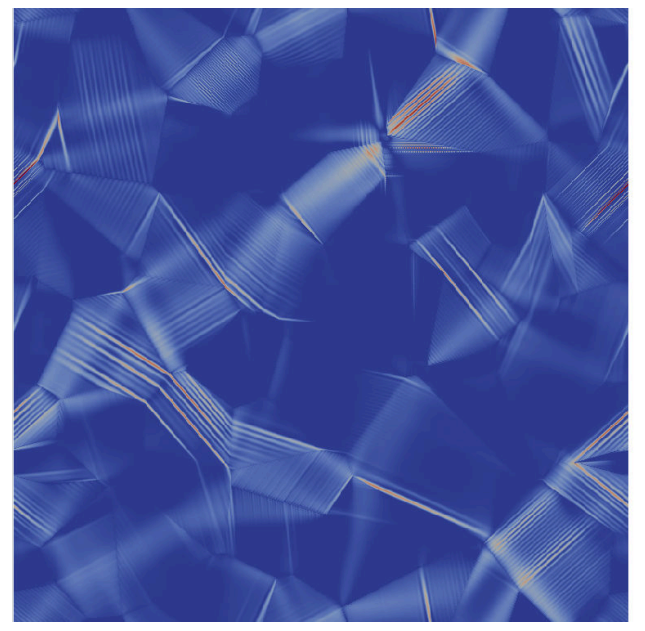

(a) continuity

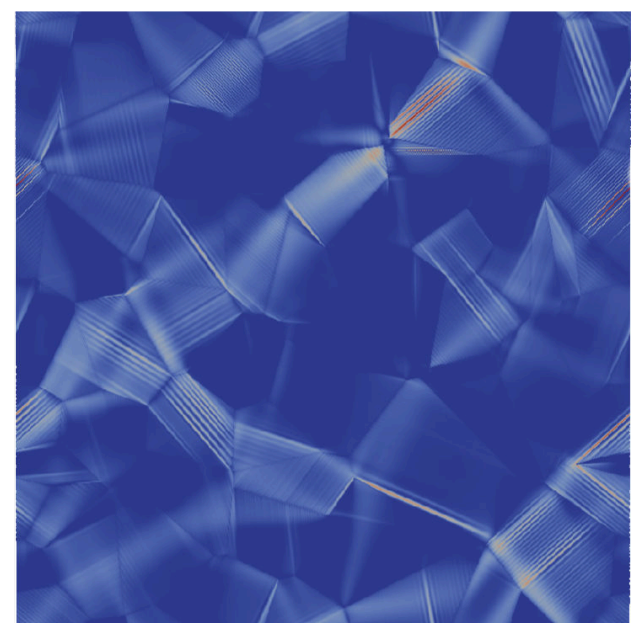

(c) microhard-2

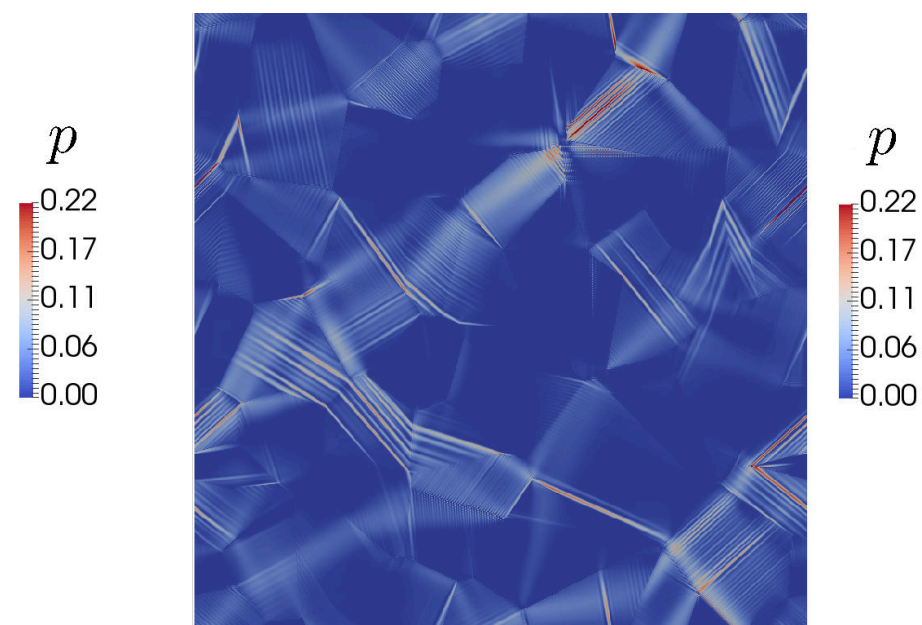

(b) microfree

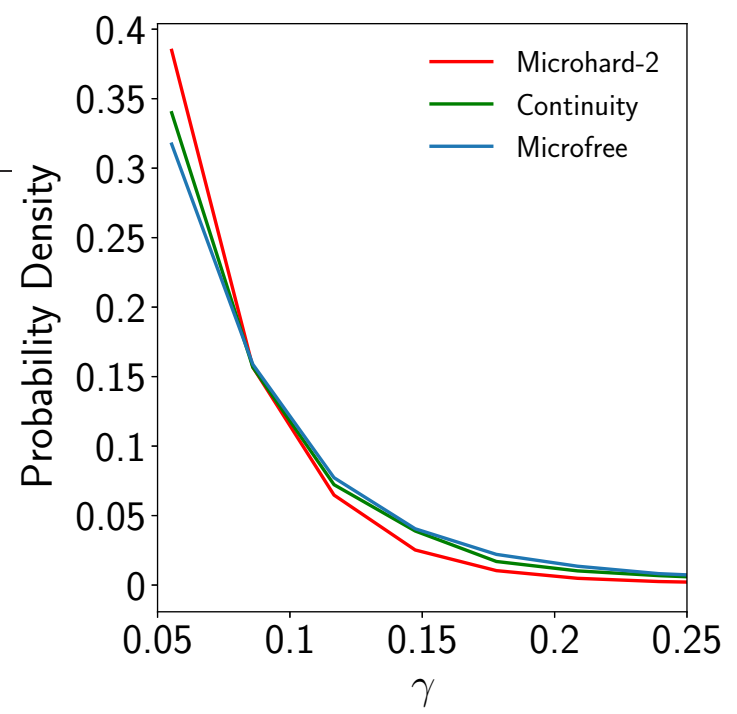

(d) microhard-1

Figure 19: Equivalent plastic strain fields for $3 \mathrm{BC}$ enforced at grain boundaries after $1 \%$ tensile strain. (d) Comparison of the equivalent plastic strain value distribution in the fields obtained with the $3 \mathrm{BC}$ conditions, considering only voxels where $p>0.05$. 64 grains polycrystal with $\frac{\lambda}{d_{g}}=0.2$ and $\frac{d_{g}}{\partial x}=100$ ( $800^{2}$ voxels).

the activation of numerous slip planes along the normal to slip plane direction, yielding a dense succession of slip bands inside the KB. On each slip plane, the GND required to generate the lattice curvature inducing the bending of atomic planes, are emitted and stacked on both sides of the band, forming dislocation wall boundaries. KBs are known to be much thicker than SBs, and this significant characteristic width is most likely linked to the length required to pile-up the GND within the KB boundaries.

It has been shown in section 2.2, under the assumptions of small elastic strain, that the lattice rotation angle $\theta$ in the KB verifies $\theta \approx \gamma$. The solutions for the slip and GND density fields that verify the assumptions of an ideal KB formation are given by eqs. (47) and (51). These analytic results show that the SGP model accounts for KBs as lattice rotation bands bounded by GND walls. Besides, according to eq. (48) the width of these rotation bands is proportional to the material length scale $\lambda$ involved in the expression of the energy 
stored by GND, which could be interpreted as their characteristic pile-up length. Hence, this SGP model theoretically captures these essential features of real KBs. The simulation results presented in section 4.2 confirm that our numerical implementation accurately reproduces these predictions.

The results of section 5 show that KBs forming within polycrystalline simulations also exhibit these characteristics. Hence, these qualitative conclusions extend beyond the ideal individual KB case. Nonetheless, KBs width observed in the SGP polycrystalline simulations show some variability. In order to study how it relates to the theoretical estimate provided by eqs. (48) and (65), the width of all KBs and SBs in the field of fig. 11 (d) have been plotted in the form of histograms presented in fig. 20 (a).

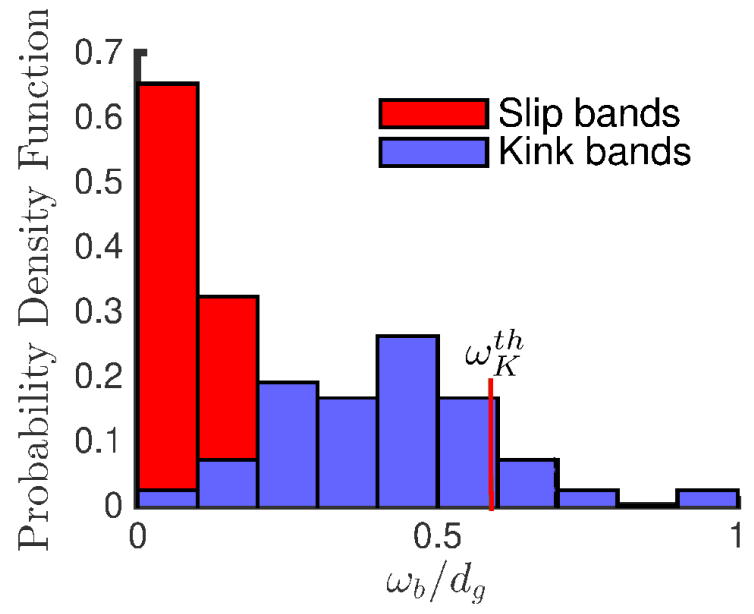

(a)

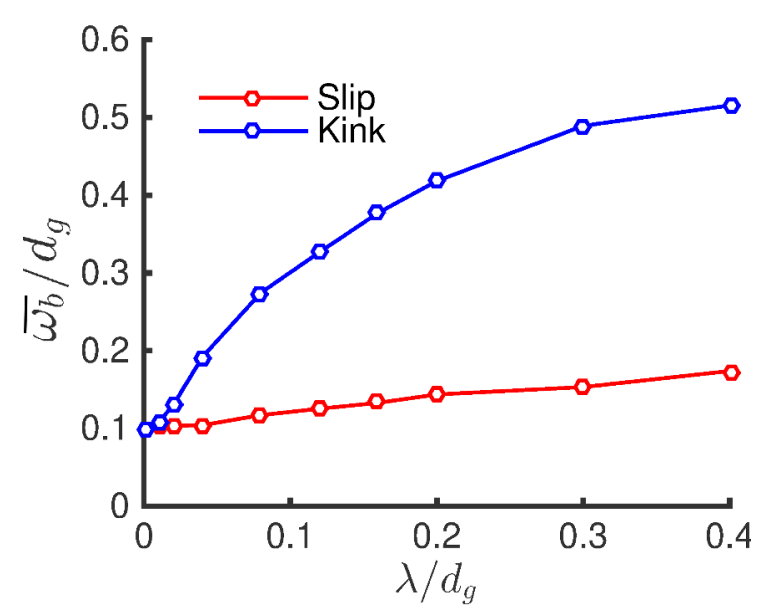

(b)

Figure 20: (a) Histograms of the normalized band width $\omega_{b} / d_{g}$ for SBs and KBs computed from the fields simulated with the SGP model, presented in fig. 11. $\omega_{K}^{t h}$ is the theoretical KB width given by eq. (65).(b) Evolution of the SBs and KBs mean band width $\bar{\omega}_{b}$ as a function of the material characteristic length $\lambda$, computed with the two-dimensional polycrystalline microstructure. $d_{g}$ is the average grain size.

The results show that the maximum of the $\mathrm{KB}$ width distribution is lower than the theoretical prediction obtained with eq. (65) (with material parameters of the second line of table 1 ), $\omega_{K}^{t h} \approx 0.59$, indicated on the histogram by the red line. However the two values are close. Observing the lattice rotation angle fields in fig. 13 and fig. 16 (d) shows that many KBs form close to grain boundaries. This could probably hinder the formation of the complete sinusoidal profile (observable in fig. 9 in the ideal case) and explain why most $\mathrm{KB}$ widths are lower than the theoretical prediction. Besides, the few high values in the upper tail of the distribution most likely correspond to two KBs forming closely in a relatively large grain and overlapping. In these situations, our threshold based post-processing would merge the two peaks, and hence consider them as one large KB. Such cases would logically yield band widths that are slightly lower than twice the theoretical size, which is consistent with the upper tail widths. All these observations confirm that the ideal $\mathrm{KB}$ modeling presented in section 2.2 provides a good quantitative estimates of the characteristics of KBs forming in polycrystalline simulations.

Hence, the model should provide a relatively precise control of the width of KBs forming in polycrystalline simulations, in particular through the value of the parameter $\lambda$. Figure 20 (b) presents the mean value of SB and $\mathrm{KB}$ width simulated with the two-dimensional polycrystalline microstructure, the material parameters of table 2 , and various values of $\lambda$. The mean KB width exhibits indeed a clear increase when $\lambda$ increases. In this case, eq. (65) predicts that for $\frac{\lambda}{d_{g}}>0.22$ the theoretical KB width is equal to the average grain size. This explains the saturation of the mean KB width for higher values of $\lambda$, as KB cannot be larger than the grain. These considerations may provide an interesting direction for the identification of the coefficients of SGP models based on Gurtin's theory, by matching the simulated KB width distribution to experimental 
measurements. However, it raises the fundamental question of the link between KB width and hardening size effects due to GND pile-ups. Indeed, most existing SGP models formulated within this framework include a single characteristic length that will simultaneously influence both phenomena. A single length scale may not be sufficient to accurately model both aspect within the same SGP model.

Note that the results presented in fig. 20 show that the SB width is only slightly affected by the value of $\lambda$, which demonstrates again the efficiency of the curlcurl* operator to ensure a vanishing backstress inside SBs (section 3.2). Besides, fig. 20 (b) shows that for $\lambda=0$, i.e. CCP, KB and SB width are equal, which is physically unrealistic. On the contrary, a clear distinction between SB and KB width appears as $\lambda$ rises, i.e. as gradient effects are stronger.

Furthermore, the plastic slip field predicted by our analytic analysis of ideal kink banding in section 2.2 is invariant along the normal to slip plane direction. Hence, it should be unable to represent the formation of KBs by the distribution of SBs along this direction observed in real materials. Yet, interestingly, the KBs formed in our numerical simulations clearly exhibit this fundamental characteristic feature of real KBs. As illustrated by fig. 8, the decomposition into a succession of SBs seems to be favored by an increased level of softening, which tends to indicate that this phenomenon is the result of a softening induced numerical instability. In this context, the slip band spacing inside the KBs is most likely grid dependent and not representative of a physical spacing. A detailed bifurcation and stability analysis of the ideal KB formation modeled here should be carried out in future works to investigate further this phenomena. Nonetheless, fig. 8 (e) demonstrates that in the presence of specific defects within the KBs, this numerical instability is mitigated: the dense succession of SBs is less pronounced while intense SBs form at these locations. Stress concentration at grain boundaries or triple junctions can play a role similar and trigger localization within KBs as these defects. In the presented polycrystalline simulations, this mechanisms could determine SB spacing inside KBs in some grains. More sophisticated non-convex SGP models, including a second length scale could be developed to induce slip band patterning along the normal to slip plane direction, or to regularize SB width. They should help to investigate further these phenomena.

\subsection{Competition between slip and kink banding}

The present authors demonstrated in a previous work (Marano et al., 2019) that with CCP models, the only factor affecting the competition between slip and kink banding are structural effects. As a result, they predict the formation of the localization bands that have the best suited orientation to accommodate the local deformation of the polycrystalline microstructure, from a purely mechanical perspective. Therefore, they can successfully predict the formation of numerous KBs within crystal structures characterized by a strong plastic anisotropy, such as hexagonal crystals deforming mainly through basal slip (see for instance fig. 12 (a)). Indeed, for such strongly anisotropic crystal structures, the very low number of available slip planes leaves kink banding as the only deformation mode available to accommodate a shear strain not aligned with the slip plane. In this case, they are effectively formed for structural reasons. On the other hand, crystal structures with a weak plastic anisotropy, like cubic structures, possess equivalent number of planes and possible orientations for slip and kink banding. Consequently, CCP model predict systematically the formation of comparable amounts of both modes in polycrystalline simulations. Yet, metallurgical observations show that kink banding is less frequent. For some physical reasons, not accounted for by CCP models, slip banding is generally favored over kink banding.

The main motivation of the present work was to introduce the energetic cost of lattice curvature formation as an additional physical factor influencing this competition, in favor of slip banding. The results presented here in section 4.1 clearly demonstrate that in the absence of structural effects influencing the SB/KB competition, accounting for this increased cost precludes kink banding. The CFC crystal structure simulations show that the picture is more complex when the microstructure influence comes into play. Indeed, a fraction of the KBs that would have form for structural reasons with a CCP model did not form when accounting for gradient effects, meanwhile significant slip banding was triggered in neighboring grains. This model is therefore effectively capable of reducing the relative amount of kink banding occuring in crystal structures 
with a low plastic anisotropy, relatively to slip banding, which is physically more sound. In addition, the observed decomposition of some KBs into a succession of parallel SBs accompanied by localization of lattice rotation, is a remarkable feature of the performed simulations also in agreement with experimental evidence of kink bands.

Nonetheless, gradient terms do not have a binary effect on localization modes competition. Structural effects can still overcome the energetic cost to trigger KB formation, as observed in a significant number of grains in the simulations. This is particularly noticeable in both the two dimensional and the 3D HCP polycrystal simulations. They feature a strong plastic anisotropy, that should strongly promote kink banding, and indeed, accounting for gradient effect in those cases did not hinder the formation of all KBs formed in the CCP case, despite the increased energetic cost. Most likely, only very high gradient effects could completely hinder KB formation. This would correspond to a characteristic width of KBs much larger than the typical grain size, and hence suggest that polycrystals with a small grain size could be KB free.

Finally, since the work presented here is dependent on the use of a locally softening model, at first glance it may seem to restrict the class of materials to which these results would apply. Nevertheless, the results show that in our simulations, materials still exhibit a macroscopic hardening behavior. This suggests that accounting for a local softening stage at incipient plasticity could improve slip localization modeling for the broader scope of hardening polycrystals. Also, this approach could be extended to study KB formation triggered by geometrical softening (late KB that arise to accommodate strain incompatibilities with only one slip system activated, typical in strongly anisotropic crystals for instance). This would require an extension to finite strains of the model and the implementation.

\section{Conclusions and future prospects}

The purpose of this work was to explore the potential of higher order plasticity theory for the modeling of plastic slip localization in polycrystals. To this end, a simple softening gradient crystal plasticity model based on Gurtin's theory has been applied to the modeling of single slip localization modes. This theory is based on the GND density tensor, a physical measure discriminating between the two single slip localization modes: as shown, it has a non-zero value inside kink bands, and vanishes over slip bands. Hence, this theory can overcome one fundamental limitation of classical crystal plasticity models, that is the equivalence between slip and kink banding (Asaro and Rice, 1977; Marano et al., 2019).

We implemented this theory within a massively parallel FFT-based solver, following the pioneering work of Lebensohn and Needleman (2016). It has been shown that the finite-difference based operator used to compute the double curl of the plastic part of the deformation gradient in their implementation does not numerically ensure a vanishing backstress in slip bands. This issue has been addressed using another finite-difference based operator able to preserve this fundamental property of the theory. Besides, various implementations of higher-order interface conditions at grain boundaries have been explored. In particular, we proposed a new strategy to implement the microhard boundary conditions, that do not rely on prescribing elastic voxels at grain boundaries, and as a result, significantly reduces the mesh-dependence of the results. Moreover, our results demonstrate that, despite their strong influence on GND induced hardening, these interface conditions affect essentially the intensity of the localization bands networks, while keeping the localization pattern.

An analytic study combined with high resolution polycrystalline simulations demonstrated that the proposed SGP model improves the physical description of single slip localization modes modeling, when compared to classical crystal plasticity models. Indeed, the model predicts the formation of kink bands as lattice rotation bands bounded by two walls of high GND density. It allows to control the width of KBs through the value of the higher-order coefficients. We also evidenced an instability that leads to the decomposition of kink bands into a dense distribution of slip bands. In the future this instability should be studied by means of stability and bifurcation analysis, and eventually addressed by more advanced gradient plasticity models introducing a new length scale to control slip band spacing within kink bands. 
Furthermore, the SGP model breaks the equivalence between slip and kink banding: contrary to classical crystal plasticity models, kink bands that are not structurally essential to the polycrystal deformation are hindered, which favors slip banding, especially for crystal structures with a low plastic anisotropy. In the end, for all these reasons, the SGP model allows to simulate plastic slip fields that are qualitatively much more representative of slip localization in real polycrystals exhibiting intense localization like irradiated or hyper-quenched metals.

In conclusion, the presented results advocate for the use of models based on a softening gradient plasticity theory relying on the GND density tensor to progress towards accurate simulation of intragranular plasticity in polycrystalline materials at incipient plasticity. In this way, an important future task will be to determine how the conclusions of the present work are influenced by a different choice of the GND energy term. The authors believe that this research direction may interestingly enrich the current debate on the formulation of strain gradient plasticity models. Indeed, this choice has been discussed by the plasticity community mainly in regard to the modeling of relevant scaling laws for grain size dependent hardening. Besides, we showed, with a simple model, that more realistic localization networks could be simulated while keeping the macroscopic stress-strain curve unchanged when compared to classical crystal plasticity simulations, by accounting for a more intense softening to compensate GND induced hardening. This suggests, as another future direction, to enhance existing crystal plasticity models with a softening stage and a GND energy term, even if these materials do not exhibit a macroscopic softening behavior. It is expected that, after re-identification of material coefficients, this will enhance the accuracy of predictions at the intragranular scale without modifying the overall behavior. Finally, extending this approach to finite strains is a promising path to improve the modeling of late kink bands formation that are triggered by geometrical softening.

\section{Appendix A. Finite differentiation curl operators applied to slip and kink bands}

Hereafter, the formula defining the 27-voxel centered FD scheme proposed by Berbenni et al. (2014) to compute partial derivatives is presented, for a regular grid of pixels of size $\left(\delta x_{1}, \delta x_{2}, \delta x_{3}\right)$. Only one term for resp. first order, unidirectional second order and bi-directional second order derivatives are given, the other terms being easily and unambiguously deduced from them:

$$
\begin{aligned}
f_{, 1}\left(i_{1}, i_{2}, i_{3}\right) & =\frac{1}{2 \delta x_{1}}\left[f\left(i_{1}+1, i_{2}, i_{3}\right)-f\left(i_{1}-1, i_{2}, i_{3}\right)\right] \\
f_{, 11}\left(i_{1}, i_{2}, i_{3}\right) & =\frac{1}{\delta x_{1}^{2}}\left[f\left(i_{1}+1, i_{2}, i_{3}\right)-2 f\left(i_{1}, i_{2}, i_{3}\right)+f\left(i_{1}-1, i_{2}, i_{3}\right)\right] \\
f_{, 12}\left(i_{1}, i_{2}, i_{3}\right)= & \frac{1}{4 \delta x_{1} \delta x_{2}}\left[f\left(i_{1}+1, i_{2}+1, i_{3}\right)-f\left(i_{1}+1, i_{2}-1, i_{3}\right)\right. \\
& \left.\quad-f\left(i_{1}-1, i_{2}+1, i_{3}\right)+f\left(i_{1}-1, i_{2}-1, i_{3}\right)\right]
\end{aligned}
$$

Here, we apply this scheme to the calculation of the curl and double curl of three particular cases of pixelized slip localization bands, shown in fig. A.21, before generalizing the results to all orientations. Three simple configurations are exhibited and studied, to illustrate the differences between the two FD formulations regarding the magnitude of the backstress computed in slip bands. However, they do not constitute a complete demonstration of this property, as other elementary band configurations could have been studied. The examples are two-dimensional to simplify the calculation. The slip fields verify $\gamma=\gamma^{*}$ inside the bands, and 0 outside. We recall that, in a two-dimensional setting, we have:

$$
\operatorname{curl}\left(\underline{\boldsymbol{H}}^{p}\right)=-\gamma_{, m} \boldsymbol{m} \otimes \boldsymbol{e}_{3} \quad \operatorname{curl}\left(\operatorname{curl}\left(\underline{\boldsymbol{H}}^{p}\right)\right)=\gamma_{, m n} \boldsymbol{m} \otimes \boldsymbol{m}-\gamma_{, m m} \boldsymbol{m} \otimes \boldsymbol{n}
$$

Besides, the directional derivatives in $\boldsymbol{m}$ and $\boldsymbol{n}$ directions of $\gamma$ are defined as follows:

$$
\begin{aligned}
\gamma_{, m} & =\gamma_{, 1} n_{2}-\gamma_{, 2} n_{1} \\
\gamma_{, m m} & =\gamma_{, 11} n_{2}^{2}+\gamma_{, 22} n_{1}^{2}-2 \gamma_{, 12} n_{1} n_{2} \\
\gamma_{, m n} & =n_{1} n_{2}\left(\gamma_{, 11}-\gamma_{, 22}\right)+\gamma_{, 12}\left(n_{2}^{2}-n_{1}^{2}\right)
\end{aligned}
$$


where $n_{1}$ and $n_{2}$ are the components of $\boldsymbol{n}$. We consider square pixels of size $\delta x$.

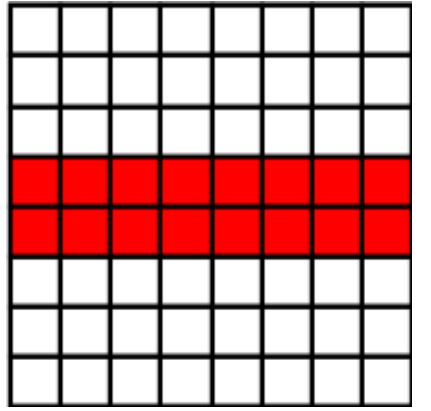

(a)

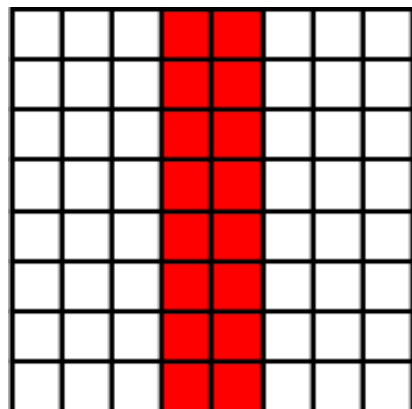

(b)

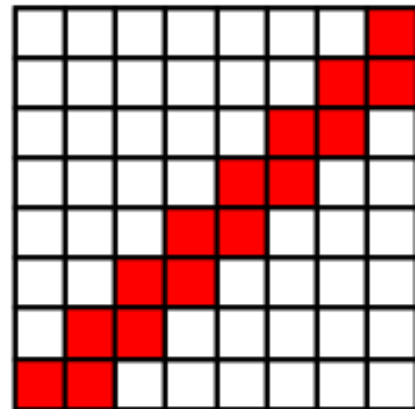

(c)

Figure A.21: Schematization of the plastic slip field of model pixelized localization bands oriented along the grid directions, with $\gamma=\gamma^{*}$ in the bands, and $\gamma=0$ outside. The components indexed by 1 are associated the horizontal direction, and indexed by 2 for the vertical direction.

\section{Horizontal band}

In the case of the horizontal band (fig. A.21-(a)), we have:

$$
\gamma_{, 1}=\gamma_{, 11}=\gamma_{, 12}=0 \quad \gamma_{, 2}= \pm \frac{\gamma^{*}}{2 \delta x} \quad \gamma_{, 22}=-\frac{\gamma^{*}}{\delta x^{2}}
$$

Then, using eqs. (A.5) to (A.7) we obtain:

$$
\gamma_{, m}= \pm \frac{n_{1}}{2 \delta x} \gamma^{*} \quad \gamma_{, m m}=-\frac{n_{1}^{2}}{\delta x^{2}} \gamma^{*} \quad \gamma_{, m n}=\frac{n_{1} n_{2}}{\delta x^{2}} \gamma^{*}
$$

The case of the slip band corresponds to $n_{1}=0$ and $n_{2}=1$, which yields

$$
\gamma_{, m}=0 \quad \gamma_{, m m}=0 \quad \gamma_{, m n}=0
$$

corresponding to $\underline{\boldsymbol{\alpha}}=0$ and $\underline{\boldsymbol{s}}=0$ (after eqs. (6) and (18). The case of the kink band corresponds to $n_{1}=1$ and $n_{2}=0$, which yields

$$
\gamma_{, m}= \pm \frac{1}{2 \delta x} \gamma^{*} \quad \gamma_{, m m}=-\frac{1}{\delta x^{2}} \gamma^{*} \quad \gamma_{, m n}=0
$$

corresponding to $\underline{\alpha} \neq 0$ and $\underline{\boldsymbol{s}} \neq 0$. Hence, here the FD scheme predicts no GND density (proportional to $\operatorname{curl}\left(\underline{\boldsymbol{H}}^{p}\right)$ ) nor backstress (proportional to $\operatorname{curl}\left(\operatorname{curl}\left(\underline{\boldsymbol{H}}^{p}\right)\right)$ ) induced by slip bands, but non-zero values for kink bands, and thus preserves exactly SB and KB physical properties. The case of the vertical band in fig. A.21(b) is exactly identical to the previous one, with interchanged indexes.

\section{5 degrees band}

We consider now the case of a $45^{\circ}$ rotated band with respect to the grid directions (fig. A.21-(c)). In this case, we have:

$$
\gamma_{, 1}= \pm \frac{1}{2 \delta x} \gamma^{*} \quad \gamma_{, 2}= \pm \frac{1}{2 \delta x} \gamma^{*} \quad \text { with } \gamma_{, 1}=-\gamma_{, 2}, \quad \gamma_{, 11}=\gamma_{, 22}=-\frac{1}{2 \delta x^{2}} \gamma^{*} \quad \gamma_{, 12}=\frac{1}{2 \delta x^{2}} \gamma^{*}
$$

Then, using eqs. (A.5) to (A.7) we obtain:

$$
\gamma_{, m}= \pm \frac{n_{1}+n_{2}}{2 \delta x} \gamma^{*} \quad \gamma_{, m m}=-\frac{1+n_{1} n_{2}}{\delta x^{2}} \gamma^{*} \quad \gamma_{, m n}=-\frac{n_{2}^{2}-n_{1}^{2}}{2 \delta x^{2}} \gamma^{*}
$$


The case of the slip band corresponds to $n_{1}=-n_{2}=-\frac{\sqrt{2}}{2}$, which yields

$$
\gamma_{, m}=0 \quad \gamma_{, m m}=-\frac{1}{2 \delta x} \gamma^{*} \quad \gamma_{, m n}=0
$$

corresponding to $\underline{\alpha}=0$ and $\underline{\boldsymbol{s}} \neq 0$.

The case of the kink band corresponds to $n_{1}=n_{2}=\frac{\sqrt{2}}{2}$, which yields

$$
\gamma_{, m}= \pm \frac{\sqrt{2}}{2 \delta x} \gamma^{*} \quad \gamma_{, m m}=-\frac{3}{2 \delta x} \gamma^{*} \quad \gamma_{, m n}=0
$$

corresponding to $\underline{\alpha} \neq 0$ and $\underline{\boldsymbol{s}} \neq 0$. Hence, here the FD scheme predicts no GND density induced by slip bands and a non-zero values for kink bands. However, it predicts a non zero backstress on the slip band $\left(\frac{1}{3}\right.$ of the kink band backstress) which is in contradiction with SB physical properties.

\section{Generic diagonal band}

A pixelized 2 pixel width localization band associated to a slip system with an arbitrary orientation in the plane, is composed of segments of the three types of band segments studied previously, and illustrated in fig. A.21, for which the expressions of $\gamma_{, m}, \gamma, m m$ and $\gamma_{, m n}$ are given above. We denote $\theta$ the angle indicating the inclination of the slip system with respect to the grid axes, so that $n_{1}=-\sin (\theta), n_{2}=\cos (\theta)$ for a slip band, and $n_{1}=\cos (\theta), n_{2}=\sin (\theta)$ for a kink band.

We then examine the case $0 \leq \theta \leq \frac{\pi}{4}$. If $\theta$ is close to $\frac{\pi}{4}$, then the band will be mostly composed of segments rotated of 45 degrees. Thus, in the case of a slip band we have:

$$
\left|\gamma_{, m}\right|_{s}=\left|\frac{\cos (\theta)-\sin (\theta)}{2 \delta x} \gamma^{*}\right| \quad\left|\gamma_{, m m}\right|_{s}=\frac{1-\frac{\sin (2 \theta)}{2}}{\delta x^{2}} \gamma^{*}
$$

and in the case of the kink band:

$$
\left|\gamma_{, m}\right|_{k}=\left|\frac{\cos (\theta)+\sin (\theta)}{2 \delta x} \gamma^{*}\right| \quad\left|\gamma_{, m m}\right|_{k}=\frac{1+\frac{\sin (2 \theta)}{2}}{\delta x^{2}} \gamma^{*}
$$

If $\theta$ is close to 0 , then the band will be mostly composed of horizontal segments. Thus, in the case of a slip band we have:

$$
\left|\gamma_{, m}\right|_{s}=\left|\frac{\sin (\theta)}{2 \delta x} \gamma^{*}\right| \quad\left|\gamma_{, m m}\right|_{s}=\frac{\sin (\theta)^{2}}{\delta x^{2}} \gamma^{*}
$$

and in the case of the kink band:

$$
|\gamma, m|_{k}=\left|\frac{\cos (\theta)}{2 \delta x} \gamma^{*}\right| \quad\left|\gamma_{, m m}\right|_{k}=\frac{\cos (\theta)^{2}}{\delta x^{2}} \gamma^{*}
$$

Accordingly, the backstress ratio between slip and kink band is:

$$
\frac{\left|\gamma_{, m m}\right|_{s}}{\left|\gamma_{, m m}\right|_{k}}=\frac{\left|1-\frac{\sin (2 \theta)}{2}\right|}{\left|1+\frac{\sin (2 \theta)}{2}\right|} \quad \text { close to } \theta=\frac{\pi}{4} \quad \frac{\left|\gamma_{, m m}\right|_{s}}{\left|\gamma_{, m m}\right|_{k}}=\frac{\left|\sin (\theta)^{2}\right|}{\left|\cos (\theta)^{2}\right|} \quad \text { close to } \theta=0
$$

and between the two in the domain $0 \leq \theta \leq \frac{\pi}{4}$. Analogous expressions can be derived for the other quadrant $\frac{\pi}{4} \leq \theta \leq \frac{\pi}{2}$. It shows that the ratio is maximal for $\gamma_{, m m}$ when $\theta=\frac{\pi}{4}$ and tends toward $\frac{1}{3}$, when it tends towards 0 for the ratio of $\gamma, m$.

This brings us to the idea of computing twice the curl with first order finite differences in order to benefit from this result in the worst case observed for the unwanted backstress on slip bands. By applying twice 
the first order FD formula on 45 degrees segments, we get an order of magnitude for the slip and then kink case:

$$
\left|\left(\gamma_{, m}\right)_{, m}\right|_{s}=\left|\frac{\cos (\theta)-\sin (\theta)}{2 \delta x}\right|^{2} \quad\left|\left(\gamma_{, m}\right)_{, m}\right|_{k}=\left|\frac{\cos (\theta)+\sin (\theta)}{2 \delta x}\right|^{2}
$$

which yields a backstress ratio of:

$$
\frac{\left|\left(\gamma_{, m}\right)_{, m}\right|_{s}}{\left|\left(\gamma_{, m}\right)_{, m}\right|_{k}}=\frac{|\cos (\theta)-\sin (\theta)|^{2}}{|\cos (\theta)+\sin (\theta)|^{2}} \quad \text { close to } \theta=\frac{\pi}{4}
$$

which tends towards 0 when $\theta$ is close to $\theta=\frac{\pi}{4}$ and is lower than $\left|1-\frac{\sin (2 \theta)}{2}\right| /\left|1+\frac{\sin (2 \theta)}{2}\right|$.

\section{References}

Acharya, A., Bassani, J., 2000. Lattice incompatibility and a gradient theory of crystal plasticity. Journal of the Mechanics and Physics of Solids 48, 1565 - 1595. doi:https ://doi.org/10.1016/S0022-5096(99)00075-7.

Anderson, D., 1965. Iterative procedures for nonlinear integral equations. J. Assoc. Comput. Mach. 12, 547-560. doi:10.1145/ 321296.321305 .

Appel, K., Haken, W., Koch, J., 1977. Every planar map is four colorable. Illinois Journal of Mathematics 21, $439-567$.

Asaro, R., Rice, J., 1977. Strain localization in ductile single crystals. Journal of the Mechanics and Physics of Solids 25, 309-338. doi:10.1016/0022-5096(77)90001-1.

Aubert, I., Saintier, N., Olive, J., 2012. Crystal plasticity computation and atomic force microscopy analysis of the internal hydrogen-induced slip localization on polycrystalline stainless steel. Scripta Materialia 66, 698-701. doi:10.1016/j. scriptamat.2012.01.019.

Bapna, M., Meshii, M., 1974. Deformation of quench-hardened gold crystals. Materials Science and Engineering $16,181-191$. doi:https ://doi .org/10.1016/0025-5416(74)90152-9.

Barton, N., Arsenlis, A., Marian, J., 2013. A polycrystal plasticity model of strain localization in irradiated iron. Journal of the Mechanics and Physics of Solids 61,341-351. doi:10.1016/j.jmps.2012.10.009.

Berbenni, S., Taupin, V., Djaka, K., Fressengeas, C., 2014. A numerical spectral appraoch for solving elasto-static field dislocation and g-disclination mechanics. International Journal of Solids and Structures 51, 4157-4175. doi:http://dx.doi. org/10.1016/j.ijsolstr.2014.08.009.

Brechet, Y., Canova, G., Kubin, L., 1993. Static versus propagative strain localisations. Scripta Metallurgica et Materialia 29, 1165-1170. doi:10.1016/0956-716X (93) 90103-Y.

Brenner, R., Beaudoin, A., Suquet, P., Acharya, A., 2014. Numerical implementation of static field dislocation mechanics theory for periodic media. Philosophical Magazine 94, 1764-1787. doi:https://doi.org/10.1080/14786435.2014.896081.

Byun, T., Hashimoto, N., Farell, K., 2006. Deformation mode map of irradiated 316 stainless steel in true stress-dose space. Journal of Nuclear Materials 351,303-315. doi:10.1016/j.jnucmat.2006.02.033.

Cermelli, P., Gurtin, M.E., 2001. On the characterization of geometrically necessary dislocations in finite plasticity. Journal of the Mechanics and Physics of Solids 49, 1539 - 1568. doi:https://doi.org/10.1016/S0022-5096(00)00084-3.

Chen, Y., Gélébart, L., Chateau, C., Bornert, M., Sauder, C., King, A., 2019. Analysis of the damage initiation in a SiC/SiC composite tube from a direct comparison between large-scale numerical simulation and synchrotron X-ray micro-computed tomography. International Journal of Solids and Structures 161, $111-126$. doi:https://doi.org/10.1016/j.ijsolstr. 2018.11.009.

Churchman, A., 1955. The yield phenomena, kink bands and geometric softening in titanium crystals. Acta Metallurgica 3, 22-29. doi:10.1016/0001-6160(55)90006-7.

Cordero, N., Gaubert, A., Forest, S., Busso, E., Gallerneau, F., Kruch, S., 2010. Size effects in generalised continuum crystal plasticity for two-phase laminates. Journal of the Mechanics and Physics of Solids 58, 1963 - 1994. doi:https: //doi.org/10.1016/j.jmps.2010.06.012.

Crone, W., Shield, T., 2001. Experimental study of the deformation near a notch tip in copper and copper-beryllium single crystals. Journal of the Mechanics and Physics of Solids 49, 2819-2938. doi:https://doi.org/10.1016/S0022-5096(01) 00080-1.

Eloh, K., Jacques, A., Berbeni, S., 2019. Development of a new consistent discrete green operator for FFT-based methods to solve heterogeneous problems with eigenstrains. International Journal of Plasticity 116, 1-23. doi:https://doi.org/10. 1016/j.ijplas.2018.10.011.

Erinosho, T., Dunne, F., 2015. Strain localization and failure in irradiated zircaloy with crystal plasticity. International Journal of Plasticity 71, 170-194. doi:10.1016/j.ijplas.2015.05.008.

Estrin, Y., Kubin, L., 1986. Local strain hardening and nonuniformity of plastic deformation. Acta Metallurgica 34, $2455-2464$. doi:https ://doi .org/10.1016/0001-6160(86)90148-3.

Fleck, N., Hutchinson, J., 1997. Strain gradient plasticity, Elsevier. volume 33 of Advances in Applied Mechanics, pp. 295 361. doi:https ://doi .org/10.1016/S0065-2156 (08)70388-0. 
Flouriot, S., Forest, S., Cailletaud, G., Köster, A., Rémy, L., Burgardt, B., Gros, V., Mosset, S., Delautre, J., 2003. Strain localization at the crack tip in single crystal CT specimens under monotonous loading: 3D finite element analyses and application to nickel-base superalloys. International Journal of Fracture 124, 44-77. doi:10.1023/B:FRAC.0000009300. 70477 . ba.

Forest, S., 1998. Modeling slip, kink and shear banding in classical and generalized single crystal plasticity. Acta Materialia 46, 3265-3281. doi:10.1016/S1359-6454(98)00012-3.

Gélébart, Ouaki, F., 2015. Filtering material properties to improve FFT-based methods for numerical homogenization. Journal of Computational Physics 294, 90 - 95. doi:https://doi.org/10.1016/j.jcp.2015.03.048.

Gélébart, L., Mondon-Cancel, R., 2013. Non-linear extension of FFT-based methods accelerated by conjugate gradients to evaluate the mechanical behavior of composite materials. Computational Materials Science 77, $430-439$. doi:https: //doi.org/10.1016/j.commatsci.2013.04.046.

Gilman, J.J., 1954. Mechanism of ortho kink-band formation in compressed zinc monocrystals. JOM 6, 621-629. doi:10.1007/ bf 03398884 .

di Gioacchino, F., da Fonseca, J.Q., 2015. An experimental study of the polycrystalline plasticity of austenitic stainless steel. International Journal of Plasticity 74, 92-109. doi:10.1016/j.ijplas.2015.05.012.

Gurtin, M.E., 2002. A gradient theory of single-crystal viscoplasticity that accounts for geometrically necessary dislocations. Journal of the Mechanics and Physics of Solids 50, 5-32. doi:https://doi.org/10.1016/S0022-5096(01)00104-1.

Hagihara, K., Mayama, T., Honnami, M., Yamasaki, M., Izuno, H., Okamoto, T., Ohashi, T., Nakano, T., Kawamura, Y., 2016a. Orientation dependence of the deformation kink band formation behavior in Zn single crystals. International Journal of Plasticity 77, 174-191. doi:10.1016/j.ijplas.2015.10.005.

Hagihara, K., Okamoto, T., Yamasaki, M., Kawamura, Y., Nakano, T., 2016b. Electron backscatter diffraction pattern analysis of the deformation band formed in the Mg-based long-period stacking ordered phase. Scripta Materialia $117,32-36$. doi:10.1016/j.scriptamat.2016.02.016.

Helfer, T., Michel, B., Proix, J.M., Salvo, M., Sercombe, J., 2015. Introducing the open-source MFront code generator: Application to mechanical behaviours and material knowledge management within the PLEIADES fuel element modelling platform. Computer and Mathematics with Applications 70, 994-1023. doi:https://doi.org/10.1016/j.camwa.2015.06. 027.

Hure, J., Shawish, S.E., Cizelj, L., Tanguy, B., 2016. Intergranular stress distributions in polycrystalline aggregates. Journal of Nuclear Materials 476, 231-242. doi:10.1016/j.jnucmat.2016.04.017.

Jaoul, B., 1961. Consolidation des polycristaux de fer et hypothèses sur l'origine du palier des courbes de traction. Journal of the Mechanics and Physics of Solids 9, 69 - 90. doi:https://doi.org/10.1016/0022-5096(61)90026-6.

Jaoul, B., 1964. Etude de la plasticité et application aux métaux. Les Presses - Mines ParisTech.

Kabel, M., Fink, A., Schneider, M., 2017. The composite voxel technique for inelastic problems. Computer Methods in Applied Mechanics and Engineering 322, 396 - 418. doi:https://doi.org/10.1016/j.cma.2017.04.025.

Kaiser, T., Menzel, A., 2019. An incompatibility tensor-based gradient plasticity formulation-theory and numerics. Computer Methods in Applied Mechanics and Engineering 345, 671 - 700. doi:https://doi.org/10.1016/j.cma.2018.11.013.

Kempe, A.B., 1879. On the geographical problem of the four colours. American journal of mathematics 2, $193-200$.

Kysar, J.W., Briant, C.L., 2002. Crack tip deformation fields in ductile single crystals. Acta Materialia 50, $2367-2380$. doi:10.1016/S1359-6454(02)00070-8.

Lebensohn, R.A., Needleman, A., 2016. Numerical implementation of non-local polycrystal plasticity using Fast Fourier transforms. Journal of the Mechanics and Physics of Solids 97, 333-351. doi:http://dx.doi.org/10.1016/j.jmps.2016.03. 023.

Ling, C., Tanguy, B., Besson, J., Forest, S., Latourte, F., 2018. A reduced micromorphic single crystal plasticity model at finite deformations. application to strain localization and void growth in ductile metals. International Journal of Solids and Structures 134, 43-69. doi:10.1016/j.ijsolstr.2017.10.013.

Mansuy, P., Philip, A., Meyssonier, J., 2001. Localization of deformation in polycrystalline ice. J. Phys. 11, 267-274. doi:http: //dx.doi.org/10.1051/jp4:2001433.

Marano, A., Gélébart, L., 2020. Non-linear composite voxels for FFT-based explicit modeling of slip bands: application to basal channeling in irradiated zr alloys. publication soumise au journal - International Journal of Solids and Structures URL: https://hal .archives-ouvertes.fr/hal-02484451.

Marano, A., Gélébart, L., Forest, S., 2019. Intragranular localization induced by softening crystal plasticity: Analysis of slip and kink bands localization modes from high resolution FFT-simulations results. Acta Materialia $175,262-275$. doi:https://doi.org/10.1016/j.actamat.2019.06.010.

Montagnat, M., Blackford, J.R., Piazolo, S., Arnaud, L., Lebensohn, R.A., 2011. Measurements and full-field predictions of deformation heterogeneities in ice. Earth and Planetary Science Letters 305, 153-160. doi:10.1016/j.epsl.2011.02.050.

Mori, T., Meshii, M., 1969. Plastic deformation of quench-hardened aluminium single crystals. Acta Metallurgica $17,167-175$. doi:10.1016/0001-6160(69) 90137-0.

Moulinec, H., Suquet, P., 1998. A numerical method for computing the overall response of nonlinear composites with complex microstructure. Comput. Methods Appl. Mech. Engrg. 157, 69-94. doi:10.1016/S0045-7825 (97)00218-1.

Neuhäuser, H., 1983. Dislocation in solids. ed. F.R.N. Nabarro. Holland Publishing Company Vol. 6,, 319-440.

Nye, J., 1953. Some geometrical relations in dislocated crystal. Acta Metallurgica 1, 153-162.

Onimus, F., Monnet, I., Béchade, J., Prioul, C., Pilvin, P., 2004. A statistical TEM investigation of dislocation channeling mechanism in neutron irradiated zirconium alloys. Journal of Nuclear Materials 328, 165-179. doi:10.1016/j.jnucmat.2004. 04.337.

Orowan, E., 1942. A type of plastic deformation new in metals. Nature 149, 643-644. doi:10.1038/149643a0. 
Patil, S.D., Narasimhana, R., Mishra, R.K., 2009. Observation of kink shear bands in an aluminium single crystal fracture specimen. Scripta Materialia 61,465-468. doi:10.1016/j.scriptamat.2009.04.043.

Patra, A., McDowell, D., 2016. Crystal plasticity investigation of the microstructural factors influencing dislocation channeling in a model irradiated bcc material. Acta Materiala 110, 364-376. doi:10.1016/j.actamat.2016.03.041.

Scherer, J., Besson, J., Forest, S., Hure, J., Tanguy, B., 2019. Strain gradient crystal plasticity with evolving length scale : Application to voided irradiated materials. European Journal of Mechanics / A Solids 77. doi:https://doi.org/10.1016/ j.euromechsol.2019.04.003.

Schneider, M., Merkert, D., Kabel, M., 2017. FFT-based homogenization for microstructures discretized by linear hexahedral elements. International journal for numerical methods in engineering 109, 1461-1489. doi:10.1002/nme.5336.

Sharp, J., 1974. Deformation of neutron irradiated copper alloys. Acta Metallurgica 22, 449-457. doi:10.1016/0001-6160 (74) 90097-2.

Steinmann, P., 1996. Views on multiplicative elastoplasticity and the continuum theory of dislocations. International Journal of Engineering Science 34, 1717 - 1735. doi:https ://doi.org/10.1016/S0020-7225(96)00062-6.

Svendsen, B., 2002. Continuum thermodynamic models for crystal plasticity including the effects of geometrically-necessary dislocations. Journal of the Mechanics and Physics of Solids 50, 1297-1329.

Willot, F., 2015a. Fourier-based schemes for computing the mechanical response of composites with accurate local fields. Comptes Rendus Mécanique 343, 232 - 245. doi:https://doi.org/10.1016/j.crme.2014.12.005.

Willot, F., 2015b. Fourier-based schemes for computing the mechanical response of composites with accurate local fields. Comptes Rendus Mécanique 343, 232-245. doi:https://doi.org/10.1016/j.crme.2014.12.005.

Wilson, C., Burg, J., Mitchell, J., 1986. The origin of kinks in polycrystalline ice. Tectonophysics 127, 27-48. doi:10.1016/ 0040-1951 (86) 90077-6.

Wulfinghoff, S., Forest, S., Böhlke, T., 2015. Strain gradient plasticity modeling of the cyclic behavior of laminate microstructures. Journal of the Mechanics and Physics of Solids 79, 1-20. doi:10.1016/j.ijplas.2014.12.003.

Xiao, X., Song, D., Xue, J., Chu, H., Duan, H., 2015. A size-dependent tensorial plasticity model for FCC single crystals with irradiation. International Journal of Plasticity 65, 152-167. doi:10.1016/j.ijplas.2014.09.004.

Zhang, M., Bridier, F., Villechaise, P., Mendez, J., McDowell, D., 2010. Simulation of slip band evolution in duplex Ti-6Al-4V. Acta Materialia 58, 1087 - 1096. doi:https://doi.org/10.1016/j.actamat.2009.10.025. 